Supporting Information for

Boron-Templated Dimerization of Allylic Alcohols to Form Protected 1,3-Diols via Acid Catalysis.

S. Hadi Nazari, Kelton G. Forson, Erin E. Martinez, Nicholas J. Hansen, K. J. Gassaway, Nathan M. Lyons, Karissa C. Kenney, Gabriel A. Valdivia-Berroeta, Stacey J. Smith, David J. Michaelis*

\title{
Table of Contents
}

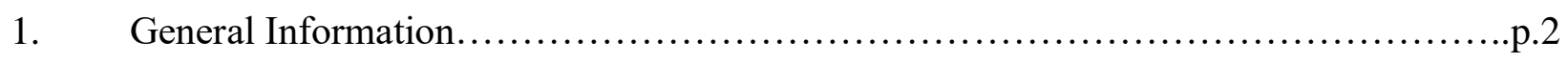

2. General Synthesis of Allylic Alcohols..........................................p.2

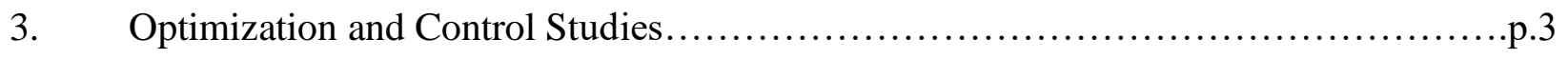

4. Synthesis of Boronic Esters Via Boron Templated Catalysis ..........................p.4

5. Derivitization Studies.................................................. 11

6. NOE Studies........................................................ 15

7. NMR Data ............................................................ 16 


\section{General Information}

All reactions were carried out under an atmosphere of nitrogen or argon in oven-dried glassware with magnetic stirring, unless otherwise indicated. Solvents were dried by J. C. Meyer's Solvent Purification System. Reactions requiring a moisture or oxygen-free environment were done in a nitrogen atmosphere glove box (Innovative Technology, PreLab HE system, double glove box). Flash chromatography was performed with Sorbtech silica gel (0.040-0.063 $\mu \mathrm{m}$ grade). Analytical thin-layer chromatography was done with $0.25 \mathrm{~mm}$ coated commercial silica gel plates (Merck KGaA, DC silicagel $60 \mathrm{~F}_{254}$ ). Proton nuclear magnetic resonance ( $1 \mathrm{H}$ NMR) data were acquired on an Inova $300(300 \mathrm{MHz})$, Inova-500 $(500 \mathrm{MHz})$ or Bruker $(500 \mathrm{MHz})$ spectrometer. Chemical shifts are reported in delta $(\delta)$ units relative to the $2 \mathrm{H}$ signal of the $\mathrm{CDCl}_{3}$ solvent. Carbon-13 and Boron-11 nuclear magnetic resonance (13C-NMR and $\left.{ }_{11} \mathrm{~B}-\mathrm{NMR}\right)$ data were acquired on an Inova 500 at $125 \mathrm{MHz}$. Signals are reported as follows: s (singlet), d (doublet), $\mathrm{t}$ (triplet), q (quartet), dd (doublet of doublets), qd (quartet of doublets), brs (broad singlet), $\mathrm{m}$ (multiplet). Coupling constants are reported in hertz (Hz). Chemical shifts are reported in ppm relative to the center line of a triplet at $77.23 \mathrm{ppm}$ for chloroform-d for $13 \mathrm{C}$ NMR or the singlet at $0 \mathrm{ppm}$ for $\mathrm{BF}_{3} \cdot \mathrm{O}(\mathrm{Et})_{2}$ for ${ }_{11} \mathrm{~B}-\mathrm{NMR}$. Infrared (IR) data were recorded as films on sodium chloride plates on a Thermo Scientific Nicolet IR 100 FT-IR spectrometer. Absorbance frequencies are reported in reciprocal centimeters (cm-1). Chiral HPLC analyses were performed on a Thermo Separation Products Spectra Series P-100 or 200 and UV100 using Chiralcel $®$ columns. Optical rotations were measured on a Jasco P-2000 digital polarimeter using $5 \mathrm{~cm}$ cells and the sodium D line $(589 \mathrm{~nm})$ at ambient temperature in the solvent and concentration indicated. Mass spectral data were obtained using ESI techniques (Agilent, 6210 TOF).

\section{General Synthesis of Allylic Alcohols}

Alcohols were prepared from aldehyde starting material, using a Horner Wadsworth Emmons reaction followed by a DIBAL reduction. An example is shown below.

To a dry flask with dry THF $(25 \mathrm{~mL})$ and sodium hydroxide $60 \%$ in mineral oil $(17.8 \mathrm{mmol})$ Triethyl phosphonoacetate $(17.8 \mathrm{mmol})$ was added dropwise. After stirring for one hour, 4-ethyl benzaldehyde $(17 \mathrm{mmol})$ was added. The reaction proceeded overnight. After workup with aqueous $\mathrm{NaHCO}_{3}$, product was purified by column chromatography with $15 \%$ ethyl acetate in hexanes as the eluent.

To a flame dried flask with dry DCM (15 mL) and ethyl (E)-3-(4-ethylphenyl)acrylate (4 mmol), DIBAL $(8.1 \mathrm{mmol})$ was added dropwise at $-78 \mathrm{C}$. The reaction was allowed to return to room temperature and continued for $2 \mathrm{~h}$. After quenching with water, the product was extracted with ethyl acetate, the organics were dried over $\mathrm{Na}_{2} \mathrm{SO}_{4}$, the solvent was removed, and the product was purified by column chromatography on silica gel with $25 \%$ ethyl acetate in hexanes as the eluent. 
Additional Optimization Studies:

\begin{tabular}{|c|c|c|c|c|c|c|}
\hline Cat. (mol\%) & Ligand & Solvent & $\mathrm{T}\left({ }^{\circ} \mathrm{C}\right)$ & Time (h) & Yield & D.R. \\
\hline $\mathrm{Cu}(\mathrm{OTf})_{2}(10)$ & Dppf (10) & Toluene & 60 & 16 & 42 & - \\
\hline $\mathrm{Cu}(\mathrm{OTf})_{2}(10)$ & - & Toluene & 60 & 16 & 24 & - \\
\hline $\mathrm{Cu}(\mathrm{OTf})_{2}(10)$ & $\mathrm{PPh} 3$ & Toluene & 60 & 16 & 43 & - \\
\hline $\mathrm{Cu}(\mathrm{OTf})_{2}(10)$ & $\mathrm{PPh} 3$ & DCE & 60 & 16 & 54 & - \\
\hline $\mathrm{Cu}(\mathrm{OTf})_{2}(10)$ & pcy3 & Toluene & 60 & 16 & 25 & - \\
\hline $\mathrm{Cu}(\mathrm{OTf})_{2}(10)$ & bipy & Toluene & 60 & 16 & 43 & - \\
\hline $\mathrm{Cu}(\mathrm{OTf})_{2}(10)$ & bipy & DCE & 60 & 16 & 47 & - \\
\hline $\mathrm{Cu}(\mathrm{OTf})_{2}(10)$ & BINAP & Toluene & 60 & 16 & 55 & - \\
\hline $\mathrm{Cu}(\mathrm{OTf})_{2}(10)$ & BINAP & Dioxane & 60 & 16 & 10 & - \\
\hline $\mathrm{Cu}(\mathrm{OTf})_{2}(10)$ & BINAP & THF & 60 & 16 & 15 & - \\
\hline $\mathrm{CuCl}_{2}(10)$ & dppf & Toluene & 60 & 16 & - & - \\
\hline $\mathrm{Cu}(\mathrm{OAC})_{2}(10)$ & dppf & Toluene & 60 & 16 & - & - \\
\hline TfOH (10) & - & Toluene & r.t & 12 & 95 & 10 to 1 \\
\hline TfOH (10) & - & DCE & r.t & 12 & 95 & 10 to 1 \\
\hline TfOH (5) & - & DCE & r.t & 12 & 30 & 10 to 1 \\
\hline TfOH (10) & - & Toluene & 60 & 12 & 90 & 10 to 1 \\
\hline
\end{tabular}

\section{Control Studies:}

To control for a hydronium effect 4 angstrom molecular sieves were added to soak up the water and reduce the potential hydronium effect. Additional studies were performed with substoichiometric phenyl boronic acid to determine the importance of equal equivalence and the acidifying effect of the phenyl boronic acid. Yields were reported based off of the phenyl boronic acid starting material. No reduction in yield was seen to indicate a controlling hydronium effect.

\begin{tabular}{|c|c|c|}
\hline $\mathrm{PhB}(\mathrm{OH})_{2}$ eq & Molecular Sieves & Yield \\
\hline 1 & no & $95 \%$ \\
\hline 0.5 & no & $96 \%$ \\
\hline 0.25 & no & $98 \%$ \\
\hline 1 & yes & $95 \%$ \\
\hline
\end{tabular}




\section{Synthesis of dioxaborinanes via boron templated catalysis}

\section{General Procedure:}

In a flame dried flask, allylic alcohol $(2 \mathrm{mmol})$ and phenyl boronic acid $(1 \mathrm{mmol})$ were stirred in toluene $(1 \mathrm{~mL})$ for 10 minutes. Triflic acid $(0.1 \mathrm{mmol})$ was added dropwise and the reaction was allowed to proceed for $12 \mathrm{~h}$. At the conclusion of the reaction, the mixture was filtered through a plug of silica gel in a pipet and rinsed through with $\mathrm{CH}_{2} \mathrm{Cl}_{2}$, the solvent was removed, and the product was loaded directly onto a column of silica gel and eluted with mixtures of ethyl acetate or $\mathrm{CH}_{2} \mathrm{Cl}_{2}$ and hexanes with $1 \%$ triethylamine to deactivate the silica gel. The

diastereoselectivity of each transformation was determined by integration of the peaks near 5-5.5 ppm corresponding to the benzylic proton of the dioxaborinane ring.

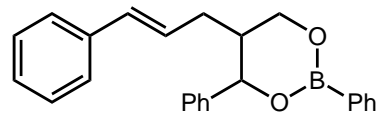

5-cinnamyl-2,4-diphenyl-1,3,2-dioxaborinane (2a): Synthesized according to the general procedure using cinnamyl alcohol $(1.5 \mathrm{~g}, 11.18 \mathrm{mmol})$, phenyl boronic acid $(0.682 \mathrm{~g}, 5.59$ mmol) and triflic acid $(83.8 \mathrm{mg})$. The reaction was purified by flash chromatography with $70 \%$ DCM $30 \%$ hexanes as the eluent, $1 \%$ triethyl amine was added to neutralize the eluent and prevent partial hydrolysis of the boronic ester. The product was isolated as a clear oil (1.9 g, 96 $\%$ yield, 14:1 DR). Rf: 0.1 in 1:1 DCM:hexanes; $1 \mathrm{H} \mathrm{NMR} \mathrm{(500} \mathrm{MHz,} \mathrm{CDCl3),} \delta 7.88$ (d, J=7.0, $2 \mathrm{H}) ; 7.50-7.21(\mathrm{~m}, 13 \mathrm{H}) ; 6.41(\mathrm{~d}, J=15.9 \mathrm{~Hz}, 1 \mathrm{H}) ; 6.09(\mathrm{td}, J=15.7 \mathrm{~Hz}, 6.7 \mathrm{~Hz}, 1 \mathrm{H}) ; 5.00$ (d, $J=7.3 \mathrm{~Hz}, 1 \mathrm{H}) ; 4.21(\mathrm{dd}, J=10.7,3.1 \mathrm{~Hz}, 1 \mathrm{H}) ; 3.99(\mathrm{dd}, J=11.8,8.0 \mathrm{~Hz}, 1 \mathrm{H}) ; 2.39-2.32(\mathrm{~m}, 1 \mathrm{H})$; 2.24-2.16 (m, 2H); $13 \mathrm{C}$ NMR (126 MHz, $\left.\mathrm{CDCl}_{3}\right), \delta 141.4,137.1,134.0,134.0,132.6,130.8$, $128.5,128.5,128.0,127.6,127.3,126.5,126.4,126.1,77.9,64.5,43.2,32.4 ; 11$ B NMR (160 $\mathrm{MHz}, \mathrm{CDCl}_{3}$ ), $\delta$ 27.0; IR (film)vmax 3057, 3027, 2903, 1950, 1600, 1312, 1261; Boron removed to gather MS data: $\mathrm{HRMS}(\mathrm{EI})$ calculated for $\mathrm{C}_{18} \mathrm{H}_{21} \mathrm{O}_{2},[\mathrm{M}+\mathrm{H}]+;$ 269.1542, found 269.1545

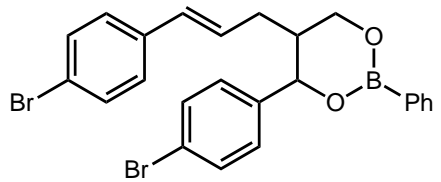

(E)-4-(4-bromophenyl)-5-(3-(4-bromophenyl)allyl)-2-phenyl-1,3,2-dioxaborinane (2b): Synthesized according to the general procedure using 4-bromo cinnamyl alcohol ( $170.4 \mathrm{mg}, 0.8$ $\mathrm{mmol})$, phenyl boronic acid $(48.8 \mathrm{mg}, 0.4 \mathrm{mmol})$ and triflic acid $(0.4 \mathrm{uL}, .04 \mathrm{mmol})$. The reaction was purified by flash chromatography with 70\% DCM 30\% hexanes as the eluent, $1 \%$ Triethyl amine was added to neutralize the eluent and prevent partial hydrolysis of the boronic ester. The product was isolated as a brown oil (204 mg, 56\%, 14:1 DR). Rf: 0.7 in DCM; $1 \mathrm{H}$ NMR (300 MHz, CDCl 3$), \delta 7.87(\mathrm{~d}, J=7.8 \mathrm{~Hz}, 2 \mathrm{H}), 7.57(\mathrm{~d}, J=8.4 \mathrm{~Hz}, 2 \mathrm{H}), 7.52-7.38(\mathrm{~m}$, $5 \mathrm{H}), 7.28(\mathrm{~d}, J=9.0 \mathrm{~Hz}, 2 \mathrm{H}), 7.18(\mathrm{~d}, J=8.4 \mathrm{~Hz}, 2 \mathrm{H}), 6.34(\mathrm{~d}, J=15.6 \mathrm{~Hz}, 1 \mathrm{H}), 6.10-6.00(\mathrm{~m}$, $1 \mathrm{H}), 4.96(\mathrm{~d}, J=6.9 \mathrm{~Hz}, 1 \mathrm{H}), 4.21(\mathrm{dd}, J=11.7,3.3 \mathrm{~Hz}, 1 \mathrm{H}), 4.02-3.96(\mathrm{~m}, 1 \mathrm{H}), 2.36-2.28(\mathrm{~m}$, $1 \mathrm{H}), 2.25-2.13(\mathrm{~m}, 2 \mathrm{H}) ; 13 \mathrm{C} \mathrm{NMR}\left(126 \mathrm{MHz}, \mathrm{CDCl}_{3}\right), \delta 140.3,135.9,134.0,131.7,131.6$, $131.0,128.4,128.2,127.7,127.6,126.9,122.0,121.1,69.9,64.5,43.0,32.3 ; 11$ B NMR (160 $\mathrm{MHz}, \mathrm{CDCl}_{3}$ ), $\delta$ 27.53; IR (film) vmax 3025, 2920, 1900, 1599, 1311, 1259, 641; HRMS(EI) calculated for $\mathrm{C}_{24} \mathrm{H}_{21} \mathrm{BBr} 2 \mathrm{O}_{2} \mathrm{Na}$, [M+Na] 534.9873, found 534.9871. 


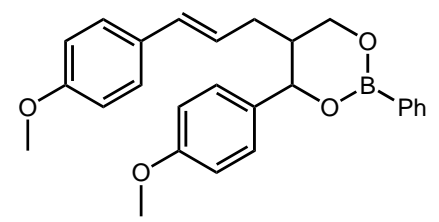

(E)-4-(4-methoxyphenyl)-5-(3-(4-methoxyphenyl)allyl)-2-phenyl-1,3,2-dioxaborinane (2c): Synthesized according to the general procedure using 4-methoxy cinnamyl alcohol (131.4 mg, $0.8 \mathrm{mmol})$, phenyl boronic acid $(48.8 \mathrm{mg}, 0.4 \mathrm{mmol})$ and triflic acid $(0.4 \mathrm{uL}, .04 \mathrm{mmol})$. The reaction was purified by flash chromatography with $100 \%$ DCM as the eluent, $1 \%$ Triethyl amine was added to neutralize the eluent and prevent partial hydrolysis of the boronic ester. The product was isolated as a yellow oil (153.6 mg, $96 \%, 10: 1 \mathrm{DR})$. Rf: 0.6 in DCM; $1 \mathrm{H}$ NMR $\left(500 \mathrm{MHz}, \mathrm{CDCl}_{3}\right), \delta 7.88(\mathrm{~d}, J=7.7 \mathrm{~Hz}, 2 \mathrm{H}) ; 7.49-7.44(\mathrm{~m}, 1 \mathrm{H}) ; 7.41-7.36(\mathrm{~m}, 2 \mathrm{H}) ; 7.31(\mathrm{~d}$, $J=8.4 \mathrm{~Hz}, 2 \mathrm{H}) ; 7.28-7.23(\mathrm{~m}, 2 \mathrm{H}) 6.96(\mathrm{~d}, J=8.4 \mathrm{~Hz}, 2 \mathrm{H}) ; 6.86(\mathrm{~d}, J=8.4 \mathrm{~Hz}, 2 \mathrm{H}) ; 6.36(\mathrm{~d}$, $J=15.5 \mathrm{~Hz}, 1 \mathrm{H}) ; 5.92(\mathrm{dt}, J=15.4 \mathrm{~Hz}, 7.9,1 \mathrm{H}) ; 4.93(\mathrm{~d}, J=8.5 \mathrm{~Hz}, 1 \mathrm{H}) ; 4.23(\mathrm{dd}, J=4.1,11.88 \mathrm{~Hz}$, $1 \mathrm{H}) ; 3.98(\mathrm{dd}, J=8.5,11.2 \mathrm{~Hz}, 1 \mathrm{H}) ; 3.85(\mathrm{~s}, 3 \mathrm{H}) ; 3.82(\mathrm{~s}, 3 \mathrm{H}) ; 2.32-2.27(\mathrm{~m}, 1 \mathrm{H}) ; 2.18-2.10(\mathrm{~m}$, 2H); $13 \mathrm{C}$ NMR (126 MHz, CDCl $), \delta$ 159.4, 159.0, 134.1, 134.0, 133.6, 131.8, 130.8, 130.0, 127.8, 127.7, 127.6, 127.3, 127.2, 127.1, 127.1, 124.2, 114.0, 113.9, 113.8, 77.7.7, 65.0, 55.3, 55.3, 46.1, 43.4, 32.3; $11 \mathrm{~B}$ NMR (160 MHz, $\mathrm{CDCl}_{3}$ ) $\delta$ 27.53; IR (film) vmax 3057, 3027, 2903, 1950, 1600, 1312, 1261; Boron removed to gather MS data HRMS(EI) calculated for $\mathrm{C}_{20} \mathrm{H}_{24} \mathrm{O} 4 \mathrm{Na}$, [M+Na]+; 351.1567, found 351.1570.<smiles>CB1OCC(C/C=C/c2ccc(C)cc2)C(c2ccc(C)cc2)O1</smiles>

(E)-2-phenyl-4-(p-tolyl)-5-(3-(p-tolyl)allyl)-1,3,2-dioxaborinane (2d):

Synthesized according to the general procedure using 4-methyl cinnamyl alcohol $(59.2 \mathrm{mg}, 0.4$ $\mathrm{mmol})$, phenyl boronic acid $(24.4 \mathrm{mg}, 0.2 \mathrm{mmol})$ and triflic acid $(0.2 \mathrm{uL}, .02 \mathrm{mmol})$. The reaction was purified by flash chromatography with $100 \%$ DCM as the eluent, $1 \%$ Triethyl amine was added to neutralize the eluent and prevent partial hydrolysis of the boronic ester. The product was isolated as a yellow oil (59.2 mg, 90.5 \%, 9:1 DR). Rf: 0.8 in DCM; $1 \mathrm{H}$ NMR (500 $\left.\mathrm{MHz}, \mathrm{CDCl}_{3}\right), \delta 7.90(\mathrm{~d}, J=7.5 \mathrm{~Hz}, 2 \mathrm{H}), 7.48(\mathrm{t}, J=7.5 \mathrm{~Hz}, 1 \mathrm{H}), 7.40(\mathrm{t}, J=7.5 \mathrm{~Hz}, 2 \mathrm{H}), 7.29$ (t, $J=7.5 \mathrm{~Hz}, 2 \mathrm{H}), 7.23(\mathrm{t}, J=7.5 \mathrm{~Hz}, 4 \mathrm{H}), 7.14(\mathrm{~d}, J=8.0 \mathrm{~Hz}, 2 \mathrm{H}), 6.38(\mathrm{~d}, J=15.5 \mathrm{~Hz}, 1 \mathrm{H})$, $6.02(\mathrm{dt}, J=8.8,15.7 \mathrm{~Hz}, 1 \mathrm{H}), 4.96(\mathrm{~d}, J=7.0 \mathrm{~Hz}, 1 \mathrm{H}), 4.23(\mathrm{dd}, J=11.5,3.5 \mathrm{~Hz}, 1 \mathrm{H}), 4.00-$ $3.97(\mathrm{~m}, 1 \mathrm{H}), 2.41$ (s, 3H), 2.36 (s, 3H), 2.21-2.14 (m, 2H); $13 \mathrm{C} \mathrm{NMR}\left(126 \mathrm{MHz}, \mathrm{CDCl}_{3}\right), \delta$ 138.5, 137.7, 137.1, 134.4, 134.1, 134.0, 132.3, 130.8, 129.3, 129.2, 129.2, 127.6, 126.5, 126.0, 125.4, 77.9, 64.7, 43.3, 32.4, 21.2; $11 \mathrm{~B}$ NMR (160 MHz, CDCl3), $\delta 27.53$; IR (film)vmax 3023, 2920, 1901, 1600, 1312, 1260; Boron removed HRMS(EI) calculated for $\mathrm{C}_{20} \mathrm{H}_{24} \mathrm{O}_{2} \mathrm{Na}$, [M+Na]+; 319.1669, found 319.1671.<smiles>CCc1ccc(/C=C/CC2COB(c3ccccc3)OC2c2ccc(CC)cc2)cc1</smiles>

(E)-4-(4-ethylphenyl)-5-(3-(4-ethylphenyl)allyl)-2-phenyl-1,3,2-dioxaborinane (2e): Synthesized according to the general procedure using 4-ethylcinnamyl alcohol (129.8 mg, 0.8 $\mathrm{mmol})$, phenyl boronic acid $(48.8 \mathrm{mg}, 0.4 \mathrm{mmol})$ and triflic acid $(0.4 \mathrm{uL}, .04 \mathrm{mmol})$. The 
reaction was purified by flash chromatography with 100\% DCM as the eluent, $1 \%$ Triethyl amine was added to neutralize the eluent and partial hydrolysis of the boronic ester. The product was isolated as a yellow oil (157 mg, $95 \%, 11: 1 \mathrm{DR})$. Rf: 0.1 in 1:1 DCM:hexanes $1 \mathrm{H}$ NMR $\left(500 \mathrm{MHz}, \mathrm{CDCl}_{3}\right), \delta 7.90(\mathrm{~d}, J=7.5 \mathrm{~Hz}, 2 \mathrm{H}), 7.47(\mathrm{t}, J=7.5 \mathrm{~Hz}, 1 \mathrm{H}), 7.40(\mathrm{t}, J=7.5 \mathrm{~Hz}, 2 \mathrm{H})$, $7.29(\mathrm{t}, J=7.5 \mathrm{~Hz}, 2 \mathrm{H}), 7.23(\mathrm{t}, J=7.5 \mathrm{~Hz}, 4 \mathrm{H}), 7.14(\mathrm{~d}, J=8.0 \mathrm{~Hz}, 2 \mathrm{H}), 6.38(\mathrm{~d}, J=15.5 \mathrm{~Hz}$, $1 \mathrm{H}), 6.02(\mathrm{dt}, J=8.8,15.7 \mathrm{~Hz}, 1 \mathrm{H}), 4.96(\mathrm{~d}, J=7 \mathrm{~Hz}, 1 \mathrm{H}), 4.23(\mathrm{dd}, J=11.5,3.5 \mathrm{~Hz}, 1 \mathrm{H}), 4.00$ $3.97(\mathrm{~m}, 1 \mathrm{H}), 2.69(\mathrm{q}, J=7.62 \mathrm{H}), 2.64(\mathrm{q}, J=7.6,2 \mathrm{H}) 2.39-2.32(\mathrm{~m}, 1 \mathrm{H}), 2.24-2.16(\mathrm{~m}, 2 \mathrm{H})$, $1.28(\mathrm{t}, J=7.5,3 \mathrm{H}), 1.24(\mathrm{t}, \mathrm{J}=7.6,3 \mathrm{H}) ;{ }_{13} \mathrm{C} \mathrm{NMR}\left(126 \mathrm{MHz}, \mathrm{CDCl}_{3}\right), \delta 138.5,137.7,137.1$, 134.4, 134.1, 134.0, 132.3, 130.8, 129.3, 129.2, 129.2, 127.6, 126.5, 126.0, 125.4, 77.9, 64.7, 43.2, 32.4, 21.2, 21.2 11B NMR (160 MHz, CDCl3), $\delta$ 27.53; IR (film)vmax 3057, 3027, 2903, 1950, 1600, 1312, 1261; Boron removed for MS. HRMS(EI) calculated For $\mathrm{C}_{22} \mathrm{H}_{28} \mathrm{O}_{2} \mathrm{Na}$, $[\mathrm{M}+\mathrm{Na}]+; 347.1982$, found 347.1980 .

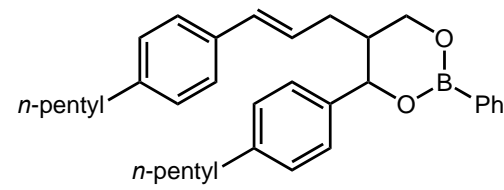

(E)-4-(4-pentylphenyl)-5-(3-(4-pentylphenyl)allyl)-2-phenyl-1,3,2-dioxaborinane (2f): Synthesized according to the general procedure using 4-pentyl cinnamyl alcohol (163.4 mg, 0.8 $\mathrm{mmol})$, phenyl boronic acid $(48.8 \mathrm{mg}, 0.4 \mathrm{mmol})$ and triflic acid $(0.4 \mathrm{uL}, .04 \mathrm{mmol})$. The reaction was purified by flash chromatography with $100 \%$ DCM as the eluent, $1 \%$ Triethyl amine was added to neutralize the eluent and prevent partial hydrolysis of the boronic ester. The product was isolated as a yellow oil $(120.6 \mathrm{mg}, 61 \%, 10: 1 \mathrm{DR})$. Rf: 0.1 in 1:1 DCM:hexanes; ${ }_{1} \mathrm{H}$ NMR (500 MHz, CDCl 3$), \delta 7.87(\mathrm{~d}, J=8.0 \mathrm{~Hz}, 2 \mathrm{H}), 7.48-7.11(\mathrm{~m}, 11 \mathrm{H}), 6.37(\mathrm{~d}, J=15.5 \mathrm{~Hz}$, $1 \mathrm{H}), 6.04-5.98(\mathrm{~m}, 1 \mathrm{H}), 4.96(\mathrm{~d}, J=7.0 \mathrm{~Hz}, 1 \mathrm{H}), 4.21(\mathrm{dd}, J=11.5,3.5 \mathrm{~Hz}, 1 \mathrm{H}), 3.97(\mathrm{dd}, J=$ 11.0, 7.5 Hz, 1H), 2.65- $2.57(\mathrm{~m}, 4 \mathrm{H}), 2.35-2.30(\mathrm{~m}, 1 \mathrm{H}), 2.20-2.15(\mathrm{~m}, 2 \mathrm{H}), 1.66-1.58(\mathrm{~m}, 4 \mathrm{H})$, 1.36-1.32 (m, 8H), 0.93-0.89 (m, 6H); $13 \mathrm{C} \mathrm{NMR} \mathrm{(126} \mathrm{MHz,} \mathrm{CDCl3),} \delta 142.8,142.2,138.6$, 134.6, 134.0, 132.3, 130.8, 128.6, 128.5, 127.6, 126.4, 126.2, 126.0, 125.9, 125.7, 125.4, 125.4, 77.9, 64.7, 43.2, 35.7, 35.6, 32.4, 31.6, 31.5, 31.4, 31.3, 31.2, 31.1, 29.7, 22.6, 22.6, 14.1, 14.0; 11B NMR (160 MHz, CDCl3), $\delta$ 25.39; IR (film)vmax 3050, 3023, 2930, 2857, 1701, 1605, 1312, 1144; HRMS(EI) calculated for $\mathrm{C}_{34} \mathrm{H}_{44} \mathrm{BO} 2$, [M+H]+; 495.3434 found 495.3432.

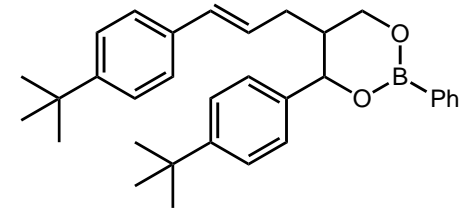

(E)-4-(4-(tert-butyl)phenyl)-5-(3-(4-(tert-butyl)phenyl)allyl)-2-phenyl-1,3,2-dioxaborinane (2g): Synthesized according to the general procedure using 4-tert butyl cinnamyl alcohol (152.2 $\mathrm{mg}, 0.8 \mathrm{mmol})$, phenyl boronic acid $(48.8 \mathrm{mg}, 0.4 \mathrm{mmol})$ and triflic acid $(0.4 \mathrm{uL}, .04 \mathrm{mmol})$. The reaction was purified by flash chromatography with $100 \%$ DCM as the eluent, $1 \%$ Triethyl amine was added to neutralize the eluent and prevent partial hydrolysis of the boronic ester. The product was isolated as a light yellow solid (87.7 $\mathrm{mg}, 47 \%, 11: 1 \mathrm{DR})$. Rf: 0.1 in 1:1 DCM:hexanes; Melting point 86-112 ${ }^{\circ} \mathrm{C} ; 1 \mathrm{H}$ NMR $\left(500 \mathrm{MHz}, \mathrm{CDCl}_{3}\right), \delta 7.87$ (d, $J=8.0 \mathrm{~Hz}$, $2 \mathrm{H}), 7.47-7.23(\mathrm{~m}, 11 \mathrm{H}), 6.37(\mathrm{~d}, J=16.0 \mathrm{~Hz}, 1 \mathrm{H}), 6.03-5.97(\mathrm{~m}, 1 \mathrm{H}), 4.96(\mathrm{~d}, J=7.0 \mathrm{~Hz}, 1 \mathrm{H})$, $4.20(\mathrm{dd}, J=11.0,3.5 \mathrm{~Hz}, 1 \mathrm{H}), 3.96$ (dd, $J=11.5,8.0 \mathrm{~Hz}, 1 \mathrm{H}), 2.37-2.31(\mathrm{~m}, 1 \mathrm{H}), 2.21-2.14$ (m, 2H), 1.34 (s, 3H), 1.32 (s, 3H); 13C NMR (126 MHz, CDCl $), \delta 150.9,150.4,138.4,134.4$, 
$134.0,132.1,130.8,127.6,126.2,125.8,125.5,125.4,77.9,69.9,64.8,43.2,34.6,34.5,32.5$, 31.4, 31.3; 11B NMR (160 MHz, CDCl3), $\delta$ 26.65; IR (film)vmax 3026, 2962, 2903, 2868, 1714, $1601,1314,1268,1143$; Boron removed for MS. HRMS(EI) calculated for $\mathrm{C}_{26} \mathrm{H}_{36} \mathrm{O}_{2} \mathrm{Na}$, $[\mathrm{M}+\mathrm{Na}]+;$ 403.2608, found 403.2609.<smiles>Clc1ccc(/C=C/CC2COB(c3ccccc3)OC2c2ccc(Cl)cc2)cc1</smiles>

(E)-4-(4-chlorophenyl)-5-(3-(4-chlorophenyl)allyl)-2-phenyl-1,3,2-dioxaborinane (2h) Synthesized according to the general procedure using 4-chloro cinnamyl alcohol (134.89 $\mathrm{mg}, 0.8$ $\mathrm{mmol})$, phenyl boronic acid $(48.8 \mathrm{mg}, 0.4 \mathrm{mmol})$, triflic acid $(0.4 \mathrm{uL}, .04 \mathrm{mmol})$ and DCE $(.4$ $\mathrm{mL}$ ) at $45 \mathrm{oC}$. The reaction was purified by flash chromatography with $50 \%$ DCM $50 \%$ hexanes as the eluent, $1 \%$ Triethyl amine was added to neutralize the eluent and prevent partial hydrolysis of the boronic ester. The product was isolated as a light-yellow oil (131 mg, 77\%, 8:1 DR). Rf: 0.7 in DCM; ${ }_{1} \mathrm{H}$ NMR $\left(300 \mathrm{MHz}, \mathrm{CDCl}_{3}\right), \delta 7.87(\mathrm{~d}, J=7.8 \mathrm{~Hz}, 2 \mathrm{H}), 7.57(\mathrm{~d}, J=8.4 \mathrm{~Hz}, 2 \mathrm{H})$, $7.52-7.38(\mathrm{~m}, 5 \mathrm{H}), 7.28(\mathrm{~d}, J=9.0 \mathrm{~Hz}, 2 \mathrm{H}), 7.18(\mathrm{~d}, J=8.4 \mathrm{~Hz}, 2 \mathrm{H}), 6.34(\mathrm{~d}, J=15.6 \mathrm{~Hz}, 1 \mathrm{H})$, 6.10-6.00 (m, 1H), $4.96(\mathrm{~d}, J=6.9 \mathrm{~Hz}, 1 \mathrm{H}), 4.21(\mathrm{dd}, J=11.7,3.3 \mathrm{~Hz}, 1 \mathrm{H}), 4.02-3.96(\mathrm{~m}, 1 \mathrm{H})$, 2.36-2.28 (m, 1H), 2.25-2.13 (m, 2H); $\left.13 \mathrm{C} \mathrm{NMR} \mathrm{(126} \mathrm{MHz,} \mathrm{CDCl}_{3}\right), \delta$ 140.3, 135.9, 134.0, $131.7,131.6,131.0,128.3,128.2,127.7,127.6,126.9,122.0,121.1,69.9,64.5,43.0,32.3 ; 11 \mathrm{~B}$ NMR (160 MHz, CDCl3), $\delta$ 27.53; IR (film) vmax 3025, 2920, 1900, 1599, 1311, 1259, 641; boron removed for MS. HRMS(EI) calculated for $\mathrm{C}_{18} \mathrm{H}_{19} \mathrm{Cl}_{2} \mathrm{O}_{2},[\mathrm{M}+\mathrm{H}]+; 337.0757$, found 337.0761

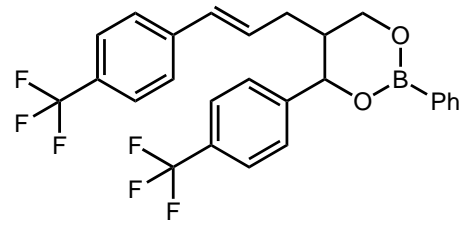

(E)-2-phenyl-4-(4-(trifluoromethyl)phenyl)-5-(3-(4-(trifluoromethyl)phenyl)allyl)-1,3,2dioxaborinane (2i):

Synthesized according to the general procedure using 4-trifluoro methyl cinnamyl alcohol (161.7 $\mathrm{mg}, 0.8 \mathrm{mmol})$, phenyl boronic acid $(48.8 \mathrm{mg}, 0.4 \mathrm{mmol})$ and triflic acid $(0.4 \mathrm{uL}, .04 \mathrm{mmol})$. The reaction was purified by flash chromatography with $100 \%$ DCM as the eluent, $1 \%$ Triethyl amine was added to neutralize the eluent and prevent partial hydrolysis of the boronic ester. The product was isolated as a yellow oil $(33.3 \mathrm{mg}, 17 \%, 10: 1 \mathrm{DR})$. Rf: 0.1 in 1:1 DCM:hexanes; $1 \mathrm{H}$ NMR (300 MHz, CDCl 3$), \delta$ 7.92-7.87 (m, 2H), $7.71(\mathrm{~d}, J=8.2 \mathrm{~Hz}, 2 \mathrm{H}) ; 7.61-7.47(\mathrm{~m}, 6 \mathrm{H})$; 7.45$7.37(\mathrm{~m}, 4 \mathrm{H}) ; 6.52-6.40(\mathrm{~m}, 1 \mathrm{H}) ; 6.16(\mathrm{dt}, J=7.0 \mathrm{~Hz}, 15.63,1 \mathrm{H}) ; 5.07(\mathrm{~d}, J=7.3 \mathrm{~Hz}, 1 \mathrm{H}) ; 4.22$ $(\mathrm{dd}, J=4.1,12.2 \mathrm{~Hz}, 1 \mathrm{H}) ; 4.07-3.98(\mathrm{~m}, 1 \mathrm{H}) ; 2.45-2.19(\mathrm{~m}, 3 \mathrm{H}) ; 13 \mathrm{C}$ NMR $(75.436 \mathrm{MHz}$, $\left.\mathrm{CDCl}_{3}\right), \delta 145.27,140.29,134.02,131.66,131.13,130.64,130.20,129.12,128.78,127.74$, 126.93, 126.26, 125.98, $125.64(\mathrm{~J}=3.79,7.38), 125.54(\mathrm{~J}=3.66,7.31), 64.40,42.98,32.34 ;{ }_{11} \mathrm{~B}$ NMR (160 MHz, CDCl3), $\delta 27.41$; IR (film)vmax 3057, 3027, 2903, 1950, 1600, 1312, 1261; Boron removed for MS. HRMS(EI) calculated for $\mathrm{C}_{20} \mathrm{H}_{19} \mathrm{~F}_{6} \mathrm{O}_{2},[\mathrm{M}+\mathrm{H}]+;$ 405.1284, found: 405.1286 


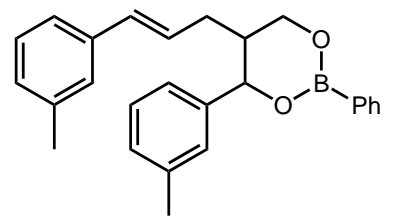

(E)-2-phenyl-4-(m-tolyl)-5-(3-(m-tolyl)allyl)-1,3,2-dioxaborinane (2j):

Synthesized according to the general procedure using 3-methyl cinnamyl alcohol (118.6 mg, 0.8 $\mathrm{mmol})$, phenyl boronic acid $(48.8 \mathrm{mg}, 0.4 \mathrm{mmol})$ and triflic acid $(0.4 \mathrm{uL}, .04 \mathrm{mmol})$. The reaction was purified by flash chromatography with $100 \%$ DCM as the eluent, $1 \%$ Triethyl amine was added to neutralize the eluent and prevent partial hydrolysis of the boronic ester. The product was isolated as a clear oil $(125 \mathrm{~g}, 82 \%, 10: 1 \mathrm{DR})$. Rf: 0.1 in 1:1 DCM:hexanes; $1 \mathrm{H}$ NMR (500 MHz, CDCl 3$), \delta 7.90(\mathrm{~d}, J=7.5 \mathrm{~Hz}, 2 \mathrm{H}), 7.48(\mathrm{t}, J=7.5 \mathrm{~Hz}, 1 \mathrm{H}), 7.40(\mathrm{t}, J=7.5 \mathrm{~Hz}$, $2 \mathrm{H}), 7.29(\mathrm{t}, J=7.5 \mathrm{~Hz}, 2 \mathrm{H}), 7.23(\mathrm{t}, J=7.5 \mathrm{~Hz}, 4 \mathrm{H}), 7.14(\mathrm{~d}, J=8 \mathrm{~Hz}, 2 \mathrm{H}), 6.38(\mathrm{~d}, J=15.5$ $\mathrm{Hz}, 1 \mathrm{H}), 6.02(\mathrm{dt}, J=8.8,15.7 \mathrm{~Hz}, 1 \mathrm{H}), 4.96(\mathrm{~d}, J=7 \mathrm{~Hz}, 1 \mathrm{H}), 4.23(\mathrm{dd}, J=11.5,3.5 \mathrm{~Hz}, 1 \mathrm{H})$, 4.00-3.97 (m, 1H), 2.41 (s, 3H), 2.36 (s, 3H), 2.21-2.14 (m, 2H); $13 \mathrm{C} \mathrm{NMR} \mathrm{(126} \mathrm{MHz,} \mathrm{CDCl} 3), \delta$ 138.5, 137.7, 137.1, 134.4, 134.1, 134.0, 132.3, 130.8, 129.3, 129.2, 129.2, 127.6, 126.5, 126.0, 125.4, 77.9, 64.7, 43.2, 32.4, 21.2; ${ }_{11 B} \mathrm{NMR}\left(160 \mathrm{MHz}, \mathrm{CDCl}_{3}\right.$ ), $\delta$ 27.53; IR (film)vmax 3023, 2920, 1901, 1600, 1312, 1260; Boron removed for MS. HRMS(EI) calculated for $\mathrm{C}_{2} \mathrm{H}_{24} \mathrm{O}_{2} \mathrm{Na}$, [M+Na]+; 319.1669, found 319.1669.<smiles>Clc1cccc(/C=C/CC2COB(c3ccccc3)OC2c2ccccc2)c1</smiles>

(E)-4-(3-chlorophenyl)-5-(3-(3-chlorophenyl)allyl)-2-phenyl-1,3,2-dioxaborinane (2k) Synthesized according to the general procedure using 3-chloro cinnamyl alcohol (134.89 mg, 0.8 $\mathrm{mmol})$, phenyl boronic acid $(48.8 \mathrm{mg}, 0.4 \mathrm{mmol})$, triflic acid $(0.4 \mathrm{uL}, .04 \mathrm{mmol})$, and DCE (.4 $\mathrm{mL})$ at $45 \mathrm{oC}$. The reaction was purified by flash chromatography with $50 \%$ DCM $50 \%$ hexanes and $1 \%$ triethylamine. The product was isolated as a light-yellow oil (101.5 mg, 60\%, 10:1 DR). Rf: 0.7 in DCM; $1 \mathrm{H}$ NMR (300 MHz, CDCl$), \delta 7.88(\mathrm{~d}, J=7.8 \mathrm{~Hz}, 2 \mathrm{H}), 7.57$ (d, $J=8.4 \mathrm{~Hz}$, $2 \mathrm{H}), 7.52-7.38(\mathrm{~m}, 5 \mathrm{H}), 7.28(\mathrm{~d}, J=9.0 \mathrm{~Hz}, 2 \mathrm{H}), 7.18(\mathrm{~d}, J=8.4 \mathrm{~Hz}, 2 \mathrm{H}), 6.34(\mathrm{~d}, J=15.6 \mathrm{~Hz}$, $1 \mathrm{H}), 6.10-6.00(\mathrm{~m}, 1 \mathrm{H}), 4.96(\mathrm{~d}, J=6.9 \mathrm{~Hz}, 1 \mathrm{H}), 4.21(\mathrm{dd}, J=11.7,3.3 \mathrm{~Hz}, 1 \mathrm{H}), 4.02-3.96(\mathrm{~m}$, $1 \mathrm{H}), 2.36-2.28(\mathrm{~m}, 1 \mathrm{H}), 2.25-2.13(\mathrm{~m}, 2 \mathrm{H}) ; 13 \mathrm{C} \mathrm{NMR}\left(126 \mathrm{MHz}, \mathrm{CDCl}_{3}\right), \delta 140.33,135.86$, 133.98, 131.68, 131.61, 131.01, 128.35, 128.25, 127.69, 127.59, 126.92, 121.98, 121.13, 69.89, 64.53, 43.04, 32.27; 11B NMR (160 MHz, CDCl3), $\delta$ 27.53; IR (film) vmax 3025, 2920, 1900 , 1599, 1311, 1259, 641; Boron removed for MS. HRMS(EI) calculated for $\mathrm{C}_{18} \mathrm{H}_{19} \mathrm{Cl}_{2} \mathrm{O}_{2}$, $[\mathrm{M}+\mathrm{H}]+; 337.0757$, found 337.0760<smiles>Cc1ccccc1/C=C/CC1COB(c2ccccc2)OC1c1ccccc1</smiles>

(E)-2-phenyl-4-(o-tolyl)-5-(3-(o-tolyl)allyl)-1,3,2-dioxaborinane (2l):

Synthesized according to the general procedure using 2-methyl cinnamyl alcohol (118.6 mg, 0.8 $\mathrm{mmol})$, phenyl boronic acid (48.8 $\mathrm{mg}, 0.4 \mathrm{mmol})$ and triflic acid $(0.4 \mathrm{uL}, .04 \mathrm{mmol})$. The 
reaction was purified by flash chromatography with $100 \%$ DCM and $1 \%$ triethylamine. The product was isolated as a clear liquid (149.2 mg, 97.6\%, 11:1 DR). Rf: 0.1 in 1:1 DCM:hexanes; 1H NMR (500 MHz, CDCl 3$), \delta 7.97(\mathrm{~d}, J=7.0 \mathrm{~Hz}, 2 \mathrm{H}) ; 7.58-7.18(\mathrm{~m}, 11 \mathrm{H}) ; 6.71(\mathrm{~d}, J=16.1$ $\mathrm{Hz}, 1 \mathrm{H}) ; 6.06(\mathrm{dt}, J=7.0 \mathrm{~Hz}, 15.64,1 \mathrm{H}) ; 5.40-5.33(\mathrm{~m}, 1 \mathrm{H}) ; 4.32(\mathrm{dd}, J=4.2,11.98 \mathrm{~Hz}, 1 \mathrm{H})$; $4.06(\mathrm{dd}, J=6.6,11.38 \mathrm{~Hz}, 1 \mathrm{H}) ; 2.49(\mathrm{~s}, 3 \mathrm{H}) ; 2.468-2.265(\mathrm{~m}, 6 \mathrm{H}) ; 13 \mathrm{C}$ NMR $(126 \mathrm{MHz}$, $\left.\mathrm{CDCl}_{3}\right), \delta 139.4,136.4,135.1,134.9,134.1,130.9,130.8,130.7,130.3,128.0,127.7,127.7$, 127.3, 126.4, 126.2, 126.1, 125.6, 64.1, 41.8, 33.0, 20.0, 19.6; ${ }_{11} \mathrm{~B}$ NMR (160 MHz, CDCl 3$), \delta$ 27.53 ; IR (film)vmax 3057, 3027, 2903, 1950, 1600, 1312, 1261; Boron removed for MS. HRMS(EI) calculated for $\mathrm{C}_{20} \mathrm{H}_{24} \mathrm{O}_{2} \mathrm{Na}$, [M+Na]+; 319.1669, found 319.1670.

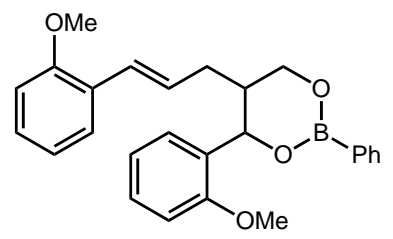

(E)-4-(2-methoxyphenyl)-5-(3-(2-methoxyphenyl)allyl)-2-phenyl-1,3,2-dioxaborinane (2m): Synthesized according to the general procedure using 2-methoxy cinnamyl alcohol (131.4 mg, $0.8 \mathrm{mmol})$, phenyl boronic acid $(48.8 \mathrm{mg}, 0.4 \mathrm{mmol})$ and triflic acid $(0.4 \mathrm{uL}, .04 \mathrm{mmol})$. The reaction was purified by flash chromatography with $100 \%$ DCM and $1 \%$ triethylamine. The product was isolated as yellow oil $(89.5 \mathrm{mg}, 54 \%, 8: 1 \mathrm{DR})$. Rf: 0.1 in 1:1 DCM:hexanes; $1 \mathrm{H}$ NMR (500 MHz, CDCl 3$), \delta 7.88(\mathrm{~d}, J=7.7 \mathrm{~Hz}, 2 \mathrm{H}) ; 7.49-7.44(\mathrm{~m}, 1 \mathrm{H}) ; 7.41-7.36(\mathrm{~m}, 2 \mathrm{H}) ; 7.31$ $(\mathrm{d}, J=8.4 \mathrm{~Hz}, 2 \mathrm{H}) ; 7.28-7.23(\mathrm{~m}, 2 \mathrm{H}) 6.96(\mathrm{~d}, J=8.4 \mathrm{~Hz}, 2 \mathrm{H}) ; 6.86(\mathrm{~d}, J=8.4 \mathrm{~Hz}, 2 \mathrm{H}) ; 6.36(\mathrm{~d}$, $J=15.5 \mathrm{~Hz}, 1 \mathrm{H}) ; 5.92(\mathrm{dt}, J=7.8,15.40 \mathrm{~Hz}, 1 \mathrm{H}) ; 4.93(\mathrm{~d}, J=8.5 \mathrm{~Hz}, 1 \mathrm{H}) ; 4.23$ (dd, $J=4.1$, $11.88 \mathrm{~Hz}, 1 \mathrm{H}) ; 3.98(\mathrm{dd}, J=8.5,11.2 \mathrm{~Hz}, 1 \mathrm{H}) ; 3.85$ (s, 3H); 3.82 (s, 3H); 2.32-2.27 (m, 1H); 2.18-2.10 (m, 2H); ${ }_{13} \mathrm{C}$ NMR (126 MHz, $\left.\mathrm{CDCl}_{3}\right), \delta$ 159.4, 159.0, 134.0, 134.0, 133.5, 131.8, $130.8,130.0,127.8,127.7,127.6,127.3,127.2$, 127.1, 127.1, 124.2, 114.0, 113.9, 113.8, 77.7.7, 64.9, 55.3, 55.3, 46.1, 43.4, 32.4; $11 \mathrm{~B}$ NMR (160 MHz, CDCl3) $\delta 27.53$; IR (film)vmax 3057, 3027, 2903, 1950, 1600, 1312, 1261; Boron removed for HRMS(EI) calculated for $\mathrm{C}_{20} \mathrm{H}_{24} \mathrm{O}_{2} \mathrm{Na}$, $[\mathrm{M}+\mathrm{Na}]+;$ 351.1567, found 351.1570.

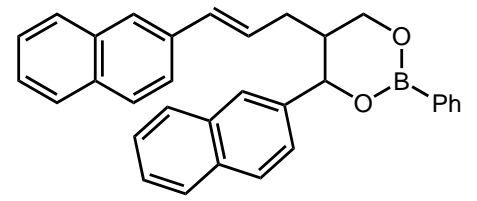

(E)-4-(naphthalen-2-yl)-5-(3-(naphthalen-2-yl)allyl)-2-phenyl-1,3,2-dioxaborinane (2n): Synthesized according to the general procedure using 3-(naphthalen-2-yl)prop-2-en-1-ol alcohol (147.4 mg, $0.8 \mathrm{mmol})$, phenyl boronic acid (48.8 mg, $0.4 \mathrm{mmol})$ and triflic acid (0.4 $\mathrm{uL}, .04$ mmol). The reaction was purified by flash chromatography with 100\% DCM and 1\% triethylamine. The product was isolated as a yellow oil $(96.3 \mathrm{mg}, 53 \%)$. Rf: 0.1 in 1:1 DCM:hexanes; $1 \mathrm{H}$ NMR $\left(500 \mathrm{MHz}, \mathrm{CDCl}_{3}\right), \delta 7.88(\mathrm{~d}, J=7.0 \mathrm{~Hz}, 2 \mathrm{H}) ; 7.50-7.21(\mathrm{~m}, 17 \mathrm{H}) ; 6.41$ $(\mathrm{d}, J=15.9 \mathrm{~Hz}, 1 \mathrm{H}) ; 6.09(\mathrm{dt}, J=15.7,6.7 \mathrm{~Hz}, 1 \mathrm{H}) ; 5.00(\mathrm{~d}, J=7.3 \mathrm{~Hz}, 1 \mathrm{H}) ; 4.21(\mathrm{dd}, J=10.7$, $3.1 \mathrm{~Hz}, 1 \mathrm{H}) ; 3.99$ (dd, $J=11.8,8.0 \mathrm{~Hz}, 1 \mathrm{H}) ; 2.39-2.32(\mathrm{~m}, 1 \mathrm{H}) ; 2.24-2.16(\mathrm{~m}, 2 \mathrm{H})$; $13 \mathrm{C} \mathrm{NMR}$ $\left(126 \mathrm{MHz}, \mathrm{CDCl}_{3}\right), \delta 141.4,137.1,134.0,134.0,132.6,130.8,128.5,128.5,128.0,127.6,127.3$, 126.5, 126.4, 126.1, 77.9, 64.5, 43.2, 32.4; ${ }_{11} \mathrm{~B}$ NMR (160 MHz, CDCl 3 ), $\delta$ 27.04; IR (film)vmax $3057,3027,2903,1950,1600,1312,1261$; HRMS(EI) calcd. for $\mathrm{C}_{32} \mathrm{H}_{28} \mathrm{BO} 2,[\mathrm{M}+\mathrm{H}]+$; 455.2177, found 455.2175. 


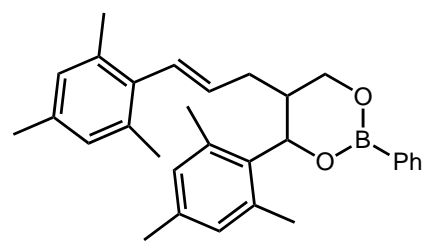

(E/Z)-4-mesityl-5-(3-mesitylallyl)-2-phenyl-1,3,2-dioxaborinane (2o):

Synthesized according to the general procedure using (E)-3-mesitylprop-2-en-1-ol (141.0 mg, $0.8 \mathrm{mmol})$, phenyl boronic acid $(48.8 \mathrm{mg}, 0.4 \mathrm{mmol})$ and triflic acid $(0.4 \mathrm{uL}, .04 \mathrm{mmol})$. The reaction was purified by flash chromatography with $100 \%$ DCM and $1 \%$ triethylamine. The product was isolated as a yellow oil as a mixture of cis and trans isomers. (144.4 g, 82\%, 8:1 DR). Rf: 0.1 in 1:1 DCM:hexanes; $1 \mathrm{H} \mathrm{NMR}\left(500 \mathrm{MHz}, \mathrm{CDCl}_{3}\right), \delta 7.98(\mathrm{~d}, J=7.0 \mathrm{~Hz}, 2 \mathrm{H})$; 7.58-7.18 (m, 5H); $6.71(\mathrm{~d}, J=16.1 \mathrm{~Hz}, 1 \mathrm{H}) ; 6.06(\mathrm{dt}, J=15.6,7.1 \mathrm{~Hz}, 1 \mathrm{H}) ; 5.40-5.33(\mathrm{~m}, 1 \mathrm{H})$; $4.32(\mathrm{dd}, J=12.0,4.2 \mathrm{~Hz}, 1 \mathrm{H}) ; 4.06(\mathrm{dd}, J=11.4,6.6 \mathrm{~Hz}, 1 \mathrm{H}) ; 2.49(\mathrm{~s}, 3 \mathrm{H}) ; 2.47-2.26(\mathrm{~m}, 18 \mathrm{H})$; ${ }_{13} \mathrm{C}$ NMR $\left(126 \mathrm{MHz}, \mathrm{CDCl}_{3}\right), \delta 139.4,136.4,135.1,134.9,134.1,130.9,130.8,130.7,130.3$, 128.0, 127.7, 127.7, 127.3, 126.4, 126.2, 126.1, 125.6, 64.1, 41.8, 33.0, 19.9, 19.6; 11 B NMR (160 MHz, CDCl3), $\delta 27.53$; IR (film)vmax 3057, 3027, 2903, 1950, 1600, 1312, 1261; Boron removed for MS. HRMS(EI) calculated For $\mathrm{C}_{24} \mathrm{H}_{32} \mathrm{O}_{2} \mathrm{Na}$, [M+Na]+; 375.2295, found 375.2293

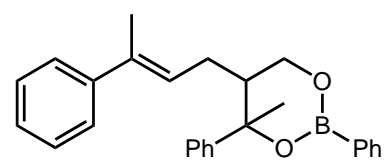

(E)-4-methyl-2,4-diphenyl-5-(3-phenylbut-2-en-1-yl)-1,3,2-dioxaborinane (2p):

Synthesized according to the general procedure using (E)-3-phenylbut-2-en-1-ol alcohol (118.6 $\mathrm{mg}, 0.8 \mathrm{mmol})$, phenyl boronic acid ( $48.8 \mathrm{mg}, 0.4 \mathrm{mmol})$ and triflic acid $(0.4 \mathrm{uL}, .04 \mathrm{mmol})$. The reaction was purified by flash chromatography with $100 \%$ DCM and $1 \%$ triethylamine. The product was isolated as a clear oil (61.2 mg, 40\%). Rf: 0.1 in 1:1 DCM:hexanes; $1 \mathrm{H}$ NMR (500 $\left.\mathrm{MHz}, \mathrm{CDCl}_{3}\right), \delta 7.95(\mathrm{~d}, J=7.0 \mathrm{~Hz}, 2 \mathrm{H}) ; 7.51-7.23(\mathrm{~m}, 13 \mathrm{H}) ; 6.71(\mathrm{~d}, J=16.1 \mathrm{~Hz}, 1 \mathrm{H}) ; 6.06$ $(\mathrm{dt}, J=15.64,7.0 \mathrm{~Hz}, 1 \mathrm{H}) ; 5.71-5.65(\mathrm{~m}, 1 \mathrm{H}) ; 4.04-3.95(\mathrm{~m}, J=4.2,11.98 \mathrm{~Hz}, 2 \mathrm{H}) ; 2.44-2.38$ (m, 1H), 2.29-2.23 (m, 2H), 1.96 (s, 3H), 1.76 (s, 3H); 13C NMR (126 MHz, CDCl $), \delta 146.72$, 143.58, 137.04, 134.04, 130.80, 128.36, 128.23, 127.70, 127.14, 126.83, 125.64, 125.26, 125.15, 63.01, 46.61, 25.75, 15.97; 11B NMR (160 MHz, CDCl3), $\delta$; IR (film)vmax 3057, 3027, 2903, 1950, 1600, 1312, 1261; Boron removed for MS. HRMS(EI) calculated For $\mathrm{C}_{20} \mathrm{H}_{24} \mathrm{O}_{2} \mathrm{Na}$, [M+Na]+; 319.1669, found 319.1668.<smiles>Fc1ccc(/C=C/CC2COB(c3ccccc3)OC2c2ccc(F)c(F)c2)cc1F</smiles>

(E)-4-(3,4-difluorophenyl)-5-(3-(3,4-difluorophenyl)allyl)-2-phenyl-1,3,2-dioxaborinane (2q):

Synthesized according to the general procedure using (E)-3-(3,4-difluorophenyl)prop-2-en-1-ol (141 mg, $0.8 \mathrm{mmol})$, phenyl boronic acid $(48.8 \mathrm{mg}, 0.4 \mathrm{mmol})$ and triflic acid $(0.4 \mathrm{uL}, .04$ $\mathrm{mmol}$ ). The reaction was purified by flash chromatography with $100 \%$ DCM and $1 \%$ triethylamine. The product was isolated as a clear oil (44.3 mg, 26\%, 10:1 DR). Rf: 0.1 in 1:1 DCM:hexanes; $1 \mathrm{H}$ NMR (500 MHz, CDCl3), $\delta 7.87(\mathrm{~d}, J=7.0 \mathrm{~Hz}, 2 \mathrm{H}), 7.47-7.23(\mathrm{~m}, 9 \mathrm{H}), 6.33$ 
$(\mathrm{d}, J=6.0 \mathrm{~Hz}, 1 \mathrm{H}), 6.05-5.95(\mathrm{~m}, 1 \mathrm{H}), 4.95(\mathrm{~d}, J=7.0 \mathrm{~Hz}, 1 \mathrm{H}), 4.21(\mathrm{dd}, J=11.0,3.5 \mathrm{~Hz}, 1 \mathrm{H})$, 3.99 (dd, $J=11.5,8.0 \mathrm{~Hz}, 1 \mathrm{H}), 2.37-2.25$ (m, 1H), 2.22-2.14 (m, 2H);13C NMR (126 MHz, $\left.\mathrm{CDCl}_{3}\right), \delta 141.4,137.1,134.0,134.0,132.6,130.8,128.5,128.0,127.6,127.3,126.5,126.4$, 126.1, 77.9, 64.5, 43.2, 32.4; $11 \mathrm{~B}$ NMR (160 MHz, $\mathrm{CDCl}_{3}$ ), $\delta$ 27.04; IR (film)vmax 3057, 3027 , 2903, 1950, 1600, 1312, 1261 Boron removed for MS. HRMS(EI) calculated for $\mathrm{C}_{18} \mathrm{H}_{16} \mathrm{~F}_{4} \mathrm{O}_{2} \mathrm{Na}$, [M+Na]+; 363.0979, found 363.0980.

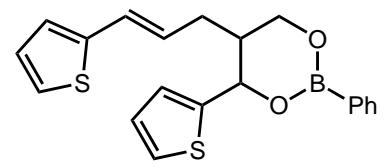

(E)-2-phenyl-4-(thiophen-2-yl)-5-(3-(thiophen-2-yl)allyl)-1,3,2-dioxaborinane (2r):

Synthesized according to the general procedure using (E)-3-(thiophen-2-yl)prop-2-en-1-ol (141 $\mathrm{mg}, 0.8 \mathrm{mmol})$, phenyl boronic acid $(48.8 \mathrm{mg}, 0.4 \mathrm{mmol})$ and triflic acid $(0.4 \mathrm{uL}, .04 \mathrm{mmol})$. The reaction was purified by flash chromatography with 100\% DCM and 1\% triethylamine. The product was isolated as a yellowish oil $(90.8 \mathrm{mg}, 62 \%, 8: 1 \mathrm{DR})$. Rf: 0.1 in 1:1 DCM:hexanes; $1 \mathrm{H}$ NMR (500 MHz, CDCl $), \delta 7.86(\mathrm{~d}, J=7.0 \mathrm{~Hz}, 2 \mathrm{H}), 7.47-7.23(\mathrm{~m}, 4 \mathrm{H}), 7.2-$ $6.8(\mathrm{~m}, 5 \mathrm{H}) 6.33(\mathrm{~d}, J=6.0 \mathrm{~Hz}, 1 \mathrm{H}), 6.05-5.95(\mathrm{~m}, 1 \mathrm{H}), 5.21(\mathrm{~d}, J=7.0 \mathrm{~Hz}, 1 \mathrm{H}), 4.21(\mathrm{dd}, J=$ $11.0,3.5 \mathrm{~Hz}, 1 \mathrm{H}), 3.99$ (dd, $J=11.5,8.0 \mathrm{~Hz}, 1 \mathrm{H}), 2.37-2.25(\mathrm{~m}, 1 \mathrm{H}), 2.22-2.14(\mathrm{~m}, 2 \mathrm{H}) ; 13 \mathrm{C}$ NMR (126 MHz, CDCl 3$), \delta 141.4,137.1,134.0,134.0,132.6,130.8,128.5,128.5,128.0,127.6$, 127.3, 126.5, 126.4, 126.1, 77.9, 64.5, 43.2, 32.4 ; 11B NMR (160 MHz, CDCl 3$), \delta 27.04$; IR (film)vmax 3057, 3027, 2903, 1950, 1600, 1312, 1261; Boron removed for MS. HRMS(EI) calculated for $\mathrm{C}_{14} \mathrm{H}_{16} \mathrm{O}_{2} \mathrm{~S}_{2} \mathrm{Na},[\mathrm{M}+\mathrm{Na}]+; 303.0484$, found 303.0485 .

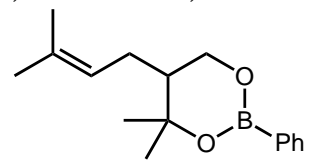

\section{4,4-dimethyl-5-(3-methylbut-2-en-1-yl)-2-phenyl-1,3,2-dioxaborinane (2s):}

Synthesized according to the general procedure using 3-methylbut-2-en-1-ol (68.9 mg, 0.8 $\mathrm{mmol})$, phenyl boronic acid $(48.8 \mathrm{mg}, 0.4 \mathrm{mmol})$ and triflic acid $(0.4 \mathrm{uL}, .04 \mathrm{mmol})$. The reaction was purified by flash chromatography with 70\% DCM 30\% hexanes and 1\% triethylamine. The product was isolated as an oil (87.8 mg, 85\%, 8:1 DR). Rf: 0.1 in 1:1 DCM:hexanes; $1 \mathrm{H}$ NMR (500 MHz, CDCl$), \delta 7.84(\mathrm{dd}, J=8.1,1.7 \mathrm{~Hz}, 2 \mathrm{H}) ; 7.51-7.33(\mathrm{~m}, 3 \mathrm{H})$; 5.23-5.14 (m, 1H); 4.13 (dd, $J=11.6,4.3 \mathrm{~Hz}, 1 \mathrm{H})$; 3.95-3.77 (m, 1H); 2.40-2.33 (m, 1H); 2.22$2.13(\mathrm{~m}, 1 \mathrm{H}) ; 1.93-1.88(\mathrm{~m}, 1 \mathrm{H}) ; 1.77(\mathrm{~s}, 3 \mathrm{H}) ; 1.67(\mathrm{~s}, 3 \mathrm{H}) ; 1.48(\mathrm{~s}, 3 \mathrm{H}) ; 1.32(\mathrm{~s}, 3 \mathrm{H}) ; 13 \mathrm{C}$ NMR $\left(126 \mathrm{MHz}, \mathrm{CDCl}_{3}\right), \delta 133.7,133.5,130.4,129.1,128.2,127.5,121.8,73.9,63.1,48.6,33.9$, 29.3, 26.4, 25.8, 23.8, 17.8; 11B NMR (160 MHz, CDCl3), $\delta$ 25.6; IR (film)vmax 3057, 3027, 2903, 1950, 1600, 1312, 1261; Boron removed for MS. HRMS(EI) calculated for $\mathrm{C}_{10} \mathrm{H}_{20} \mathrm{O}_{2} \mathrm{Na}$, $[\mathrm{M}+\mathrm{Na}]+;$ 195.1356, found 195.1351.

\section{Derivatization Studies}

\section{2-cinnamyl-1-phenylpropane-1,3-diol (3)}<smiles>OCC(CC=Cc1ccccc1)C(O)c1ccccc1</smiles>

In a vial 5-cinnamyl-2,4-diphenyl-1,3,2-dioxaborinane $(0.4 \mathrm{mmol}, 141.7 \mathrm{mg})$ and sodium hydroxide $(2 \mathrm{mmol}, 80 \mathrm{mg})$ in a 1:1 solution of THF: $\mathrm{H}_{2} \mathrm{O}(8 \mathrm{~mL})$ was stirred at $60{ }^{\circ} \mathrm{C}$ for $20 \mathrm{~h}$. 
The product was then extracted from the mixture with DCM, and the combined organics were dried over sodium sulphate. The product was isolated as an oil (107.3 mg, 100\%). Rf: 0.1 in 1:1 DCM:hexanes; ${ }_{1 H} \mathrm{NMR}\left(500 \mathrm{MHz}, \mathrm{CDCl}_{3}\right), \delta 7.50-7.21(\mathrm{~m}, 10 \mathrm{H}) ; 6.41(\mathrm{~d}, J=15.9 \mathrm{~Hz}, 1 \mathrm{H})$; 6.09 (dt, $J=15.7,6.7 \mathrm{~Hz}, 1 \mathrm{H}) ; 5.00(\mathrm{~d}, J=7.3,1 \mathrm{H}) ; 4.21(\mathrm{dd}, J=10.73,3.12 \mathrm{~Hz}, 1 \mathrm{H}) ; 3.99$ (dd, $J$ $=11.77,7.96 \mathrm{~Hz}, 1 \mathrm{H}) ; 2.393-2.320(\mathrm{~m}, 1 \mathrm{H}) ; 2.245-2.163(\mathrm{~m}, 2 \mathrm{H}) ; 13 \mathrm{C} \mathrm{NMR}\left(126 \mathrm{MHz}, \mathrm{CDCl}_{3}\right)$, $\delta 141.4,137.1,134.0,134.0,132.6,130.8,128.5,128.5,128.0,127.6,127.3,126.5,126.4,126.1$, 77.9, 64.5, 43.2, 32.4 11B NMR (160 MHz, CDCl3), $\delta$ 27.04; IR (film)vmax 3057, 3027, 2903, $1950,1600,1312,1261 ; \mathrm{HRMS}(\mathrm{EI})$ calculated for $\mathrm{C}_{18} \mathrm{H}_{21} \mathrm{O}_{2}$, [M+H]+; 268.1463, found 268.1725

\section{Dicinnamyl phenylboronate (4)}

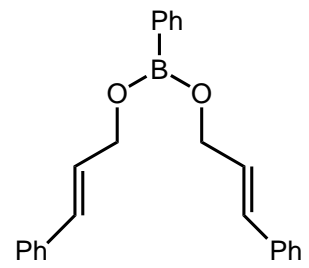

In a dry vial cinnamyl alcohol $(0.8 \mathrm{mmol}, 107.4 \mathrm{mg})$ and phenyl boronic acid $(0.4 \mathrm{mmol}, 48.8$ $\mathrm{mg}$ ) were stirred in toluene $(0.4 \mathrm{~mL})$ for $10 \mathrm{~min}$ at room temperature. The product was achieved in quantitative yield and used for the following reaction. The product was isolated as a clear oil (141.7 mg, >99\%). $1 \mathrm{H}$ NMR (500 MHz, CDCl $), \delta 7.85(\mathrm{~d}, J=7.2 \mathrm{~Hz}, 1 \mathrm{H}) ; \delta 7.68(\mathrm{~d}, J=5.9$ $\mathrm{Hz}, 1 \mathrm{H}) ; \delta$ 7.43-7.17 (m, $15 \mathrm{H}) ; \delta$ 6.67-6.57 (m, $2 \mathrm{H}) ; \delta$ 6.38-6.26 (m, $2 \mathrm{H}) ; \delta 4.71(\mathrm{~d}, 4 \mathrm{H}){ }_{13} \mathrm{C}$ NMR (126 MHz, $\left.\mathrm{CDCl}_{3}\right), \delta$ 137.0, 134.8, 133.6, 131.5, 130.7, 130.1, 128.7, 128.1, 128.0, 127.7, 126.6; HRMS(EI) calculated for $\mathrm{C}_{24} \mathrm{H}_{24} \mathrm{BO} 2$, [M+H]+ 355.1864, found 355.1861

\section{bis(cinnamyloxy)dimethylsilane (5)}

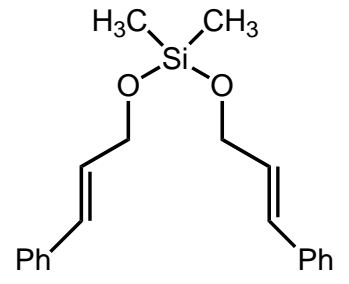

In a dry vial, cinnamyl alcohol $(7.5 \mathrm{mmol}, 1.026 \mathrm{~g})$ and triethylamine $(7.8 \mathrm{mmol}, 1.1 \mathrm{~mL})$ were stirred for 5 minutes at room temperature in dichloromethane $(8 \mathrm{~mL})$. Dimethyldichlorosilane $(3.7 \mathrm{mmol}, 0.45 \mathrm{~mL})$ was added slowly and the reaction was stirred for 4 hours at room temperature. The solution was concentrated, washed with pentane, and filtered. The reaction was purified by flash chromatography with $5 \%$ methanol in dichloromethane. The product was isolated as an oil $(817 \mathrm{mg}, 68 \%)$. Characterization matched literature by Fleming, (Tet. Lett. 1992, 33, 1013-1016). $1 \mathrm{H} \mathrm{NMR} \mathrm{(500} \mathrm{MHz,} \mathrm{CDCl3):} \delta=7.38$ (d, $J=8.5 \mathrm{~Hz}, 4 \mathrm{H}), 7.31$ (t, $J=$ $8.0 \mathrm{~Hz}, 4 \mathrm{H}), 7.25-7.23$ (m, $2 \mathrm{H}), 6.63-6.59(\mathrm{~m}, 2 \mathrm{H}), 6.36-6.29$ (m, $2 \mathrm{H}), 4.45-4.44$ (dd, $J=1.5$, $5.5 \mathrm{~Hz}, 3 \mathrm{H}), 4.43-4.41(\mathrm{dd}, J=1.5,5.5 \mathrm{~Hz}, 1 \mathrm{H}), 0.26(\mathrm{~s}, 4 \mathrm{H}), 0.20$ (s, $2 \mathrm{H})$.<smiles>OPc1ccc2ccccc2c1-c1c(P(O)O)ccc2ccccc12</smiles>

(2'-phenyl-[1,1'-binaphthalen]-2-yl)boronic acid (6) 
Prepared following the procedure by Hisashi (Synthesis, 2017, 49, 175-180). A mixture of 1,1'binaphthyl-2,2'-diboronic acid (3, $171 \mathrm{mg}, 0.5 \mathrm{mmol}, 1$ equiv), $\mathrm{Pd}(\mathrm{PPh} 3) 4$ (14.4 mg, $2.5 \mathrm{~mol} \%)$, $\mathrm{Ba}(\mathrm{OH})_{2} \cdot 8 \mathrm{H}_{2} \mathrm{O}(315.5 \mathrm{mg}, 1 \mathrm{mmol}, 2$ equiv), and iodobenzene ( $56 \mu \mathrm{L}, 0.5 \mathrm{mmol}, 1$ equiv) in THF $(5 \mathrm{~mL})$ and water $(1 \mathrm{~mL})$ was heated at $60^{\circ} \mathrm{C}$ for $14 \mathrm{~h}$. The mixture was then allowed to cool down to r.t. The residue was extracted with $\mathrm{CH}_{2} \mathrm{Cl}_{2}$, washed with brine and dried $\left(\mathrm{Na}_{2} \mathrm{SO}_{4}\right)$. After filtration and removal of solvent, the crude product was purified by column chromatography (hexane/EtOAc 4:1) to afford the product (136 mg, 73\%) as a white solid. The enantiomeric excess of $\mathbf{6}$ was determined to be 96.4 percent ee by HPLC (Hexanes/iPrOH 80:20, flow rate $=1.0 \mathrm{~mL} / \mathrm{min}, \lambda=254 \mathrm{~nm}, \mathrm{~T}=20^{\circ} \mathrm{C}$ ). Spectral data matched reported literature.

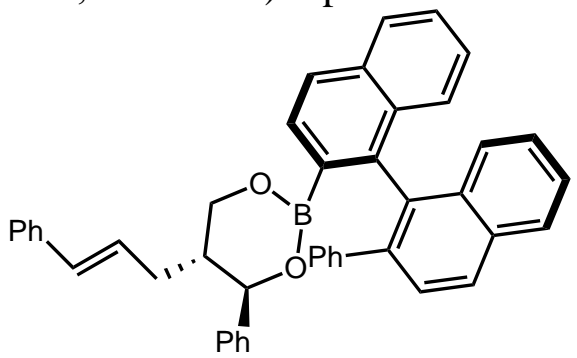

$(4 R, 5 R)-5-c i n n a m y l-4-p h e n y l-2-((R)-2 '-p h e n y l-[1,1 '-b i n a p h t h a l e n]-2-y l)-1,3,2-$ dioxaborinane (7)

In a flame dried vial cinnamyl alcohol $(0.748 \mathrm{mmol}, 100 \mathrm{mg})$ and 2'-phenyl-[1,1'-binaphthalen]2-yl)boronic acid $(0.374 \mathrm{mmol}, 140 \mathrm{mg})$ were stirred in toluene $(0.4 \mathrm{~mL})$ at room temperature for $10 \mathrm{~min}$. Triflic acid $(0.0374 \mathrm{mmol}, 5.6 \mathrm{mg})$ was added and the reaction continued for $8 \mathrm{~h}$. The reaction was purified by flash chromatography with 70\% DCM 30\% hexanes and 1\% triethylamine. The product was isolated as an oil. (162.2 mg, 71.4\%, 1.2:1 DR) $1 \mathrm{H} \mathrm{NMR} \mathrm{(500}$ $\left.\mathrm{MHz}, \mathrm{CDCl}_{3}\right), \delta 7.92(\mathrm{~d}, J=7.0 \mathrm{~Hz}, 1 \mathrm{H}) ; 7.50-7.21(\mathrm{~m}, 23 \mathrm{H}) ; 6.41$ (d, J=15.9 Hz, 1H); 6.09 (td, $J=15.7,6.7 \mathrm{~Hz}, 1 \mathrm{H}) ; 5.00(\mathrm{~d}, J=7.3 \mathrm{~Hz}, 1 \mathrm{H}) ; 4.21(\mathrm{dd}, J=10.7,3.1 \mathrm{~Hz}, 1 \mathrm{H}) ; 3.99$ (dd, $J=11.8,7.9$ $\mathrm{Hz}, 1 \mathrm{H}) ; 2.39-2.32$ (m, 1H); 2.24-2.16 (m, 2H); $13 \mathrm{C} \mathrm{NMR} \mathrm{(126} \mathrm{MHz,} \mathrm{CDCl} 3), \delta 141.4,137.1$, 134.0, 134.0, 132.6, 130.8, 128.5, 128.5, 128.0, 127.6, 127.3, 126.5, 126.4, 126.1, 77.9, 64.5, 43.2, $32.4 ; 11 \mathrm{~B}$ NMR (160 MHz, $\mathrm{CDCl}_{3}$ ), $\delta$ 27.04; IR (film)vmax 3057, 3027, 2903, 1950, 1600, 1312, 1261; HRMS(EI) calculated $\mathrm{C}_{44} \mathrm{H}_{35} \mathrm{BnaO}_{2}{ }^{+},[\mathrm{M}+\mathrm{Na}]_{+} ; 629.2622$, found 629.2619

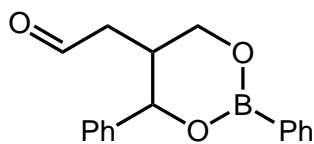

\section{2-(2,4-diphenyl-1,3,2-dioxaborinan-5-yl)acetaldehyde (8)}

In a dry vial 5-cinnamyl-2,4-diphenyl-1,3,2-dioxaborinane $(0.28 \mathrm{mmol}, 100 \mathrm{mg})$ and $2.5 \% \mathrm{wt}$ Osmium tetroxide $(0.0043 \mathrm{mmol})$ were dissolved in $0.6 \mathrm{~mL}$ THF: $\mathrm{H}_{2} \mathrm{O} 3: 1$ solution. Sodium periodate $(0.56 \mathrm{mmol}, 120 \mathrm{mg})$ was added slowly and the reaction proceeded stirring at room temperature for two hours. The reaction was quenched with sodium bicarbonate, extracted with $\mathrm{CH}_{2} \mathrm{Cl}_{2}$ and then purified by flash chromatography. The product was isolated as an oil (77 $\mathrm{mg}$, 98.2\%, 14:1 DR). Rf: 0.1 in 1:1 DCM:hexanes; $1 \mathrm{H}$ NMR (500 MHz, $\left.\mathrm{CDCl}_{3}\right), \delta 9.62(\mathrm{~s}, 1 \mathrm{H}), \delta$ $7.91(\mathrm{~d}, J=7.0 \mathrm{~Hz}, 2 \mathrm{H}), 7.47-7.30(\mathrm{~m}, 10 \mathrm{H}), 5.01(\mathrm{~d}, J=7.2 \mathrm{~Hz}, 1 \mathrm{H}), 4.20(\mathrm{dd}, J=11.0,4.0 \mathrm{~Hz}$, $1 \mathrm{H}), 3.95-3.90(\mathrm{~m}, 1 \mathrm{H}), 2.73-2.68(\mathrm{~m}, 1 \mathrm{H}), 2.66-2.46(\mathrm{~m}, 2 \mathrm{H}) ; 13 \mathrm{C} \mathrm{NMR}\left(126 \mathrm{MHz}, \mathrm{CDCl}_{3}\right), \delta$ 199.5, 140.7, 134.2, 131.1, 128.8, 128.3, 127.6, 126.4, 64.2, 43.0, 37.6; 11 B NMR (160 MHz, $\mathrm{CDCl}_{3}$ ), $\delta$ 27.04; IR (film) vmax 3057, 3027, 2903, 1950, 1700, 1312, 1261; HRMS(EI) calculated. for $\mathrm{C}_{17} \mathrm{H}_{17} \mathrm{O} 3 \mathrm{~B},[\mathrm{M}+\mathrm{H}]+; 280.1271$, found 280.1702 


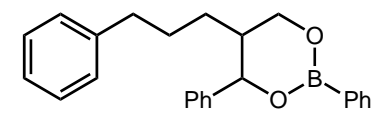

\section{2,4-diphenyl-5-(3-phenylpropyl)-1,3,2-dioxaborinane (9)}

In a flame dried vial with 5-cinnamyl-2,4-diphenyl-1,3,2-dioxaborinane $(0.4235 \mathrm{mmol}, 150 \mathrm{mg})$ and $10 \%$ palladium on carbon $(20 \mathrm{mg})$ were stirred in methanol $(4 \mathrm{~mL})$ for $7 \mathrm{~h}$ while $\mathrm{H}_{2}$ gas was bubbled through. Product was purified by column chromatography. The product was isolated as an oil (137.9 mg, 91\%, 14:1 DR). Rf: 0.1 in 1:1 DCM:hexanes; ${ }_{1} \mathrm{H} \mathrm{NMR} \mathrm{(500} \mathrm{MHz,} \mathrm{CDCl3),} \delta$ 7.88 (d, J=7.0 Hz, 2H); 7.50-7.21 (m, 15H); $4.93(\mathrm{~d}, \mathrm{~J}=7.29 \mathrm{~Hz}, 1 \mathrm{H}) ; 4.21$ (dd, $J=10.73,3.12 \mathrm{~Hz}$, $1 \mathrm{H}) ; 3.99$ (dd, $J=11.8,8.0 \mathrm{~Hz}, 1 \mathrm{H}) ; 2.39-2.32(\mathrm{~m}, 2 \mathrm{H}) ; 2.24-2.16$ (m, 3H); $13 \mathrm{C}$ NMR (126 MHz, $\left.\mathrm{CDCl}_{3}\right), \delta 141.4,137.1,134.0,134.0,132.6,130.8,128.5,128.5,128.0,127.6,127.3,126.5$, 126.4, 126.1, 77.9, 64.5, 43.2, 32.4; ${ }_{11 \mathrm{~B}} \mathrm{NMR}\left(160 \mathrm{MHz}, \mathrm{CDCl}_{3}\right.$ ), $\delta$ 27.04; IR (film)vmax 3057, 3027, 2903, 1950, 1600, 1312, 1261; Boron removed HRMS(EI) calculated for $\mathrm{C}_{24} \mathrm{H}_{25} \mathrm{BO}$, $[\mathrm{M}+\mathrm{H}]+; 356.1948$, found 356.1760

\section{5-allyl-2,4-diphenyl-1,3,2-dioxaborinane (10)}

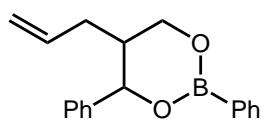

In a flame dry vile with 5-cinnamyl-2,4-diphenyl-1,3,2-dioxaborinane (2a) $(.28 \mathrm{mmol})$ and Hoveyda Grubbs 2 nd generation catalyst $(0.02 \mathrm{mmol})$ in DCE $(10 \mathrm{~mL})$ Ethylene gas was bubbled through via a balloon and syringe. After $48 \mathrm{~h}$ crude product was purified by column chromatography with 1:1 DCM:hexanes as the eluent. The product was isolated as an oil (56.4 $\mathrm{mg}, 72 \%, 14: 1 \mathrm{DR})$. Rf: 0.1 in 1:1 DCM:hexanes; $1 \mathrm{H} \mathrm{NMR}\left(500 \mathrm{MHz}, \mathrm{CDCl}_{3}\right), \delta 7.89$ (d, $J=7.0$ $\mathrm{Hz}, 2 \mathrm{H}) ; 7.50-7.34(\mathrm{~m}, 8 \mathrm{H}) ; 5.78-5.68(\mathrm{~m}, 1 \mathrm{H}) ; 5.12-5.05(\mathrm{~m}, 2 \mathrm{H}) ; 4.96(\mathrm{~d}, J=7.4 \mathrm{~Hz}, 1 \mathrm{H}) ; 4.18$ (dd, $J=11.9,4.0 \mathrm{~Hz}, 1 \mathrm{H}) ; 3.96-3.91(\mathrm{~m}, 1 \mathrm{H}) ; 2.25-2.18(\mathrm{~m}, 1 \mathrm{H}) ; 2.15-2.00(\mathrm{~m}, 2 \mathrm{H}) ; 13 \mathrm{C}$ NMR $\left(126 \mathrm{MHz}, \mathrm{CDCl}_{3}\right), \delta$ 141.4, 134.7, 134.0, 130.9, 128.5, 127.9, 127.6, 126.5, 117.5, 77.9, 64.6, 42.6, 33.1; $11 \mathrm{~B}$ NMR (160 MHz, $\mathrm{CDCl}_{3}$ ), $\delta$ 27.04; IR (film)vmax 3057, 3027, 2903, 1950, 1600, 1312,1261 ;

HRMS(EI) calculated. for $\mathrm{C}_{18} \mathrm{H}_{19} \mathrm{BO}_{2}$, [M+H]+; 278.1478, found 278.2058.

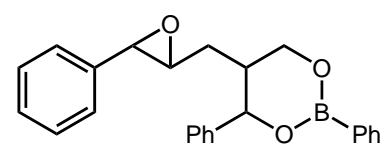

\section{2,4-diphenyl-5-((3-phenyloxiran-2-yl)methyl)-1,3,2-dioxaborinane (11)}

Dimethyldioxirane (DMDO) was prepared following the procedure in Org. Synth. 2013, 90, 350357. In a vial with 5-cinnamyl-2,4-diphenyl-1,3,2-dioxaborinane (100 $\mathrm{mg}, 0.2823 \mathrm{mmol})$ and DMDO $(0.3105 \mathrm{mmol})$ in $10 \mathrm{~mL}$ acetone was stirred for $16 \mathrm{~h}$ at room temperature. Solvent was removed under reduced pressure and purified by chromatography with 70:30 DCM hexane as the eluent. Product was purified as a 1:1 ratio of diastereomers. The product was isolated as an oil (73.6 mg, 71.4\%, 14:1 DR). Rf: 0.1 in 1:1 DCM:hexanes; $\left.{ }_{1} \mathrm{H} \mathrm{NMR} \mathrm{(500} \mathrm{MHz,} \mathrm{CDCl}_{3}\right), \delta 7.89$ $(\mathrm{d}, \mathrm{J}=7.0 \mathrm{~Hz}, 2 \mathrm{H}) ; 7.50-7.18(\mathrm{~m}, 15 \mathrm{H}) ; 5.00(\mathrm{dd}, \mathrm{J}=7.3 \mathrm{~Hz}, 1 \mathrm{H}) ; 4.43-4.26(\mathrm{~m}, 1 \mathrm{H}) ; 4.07(\mathrm{~d}$, $\mathrm{J}=44.6 \mathrm{~Hz}, 1 \mathrm{H}) ; 2.92-2.85(\mathrm{~m}, 1 \mathrm{H}) ; 2.38-2.29(\mathrm{~m}, 1 \mathrm{H}) ; 1.95-1.81(\mathrm{~m}, 1 \mathrm{H}) ; 1.71-1.44(\mathrm{~m}, 2 \mathrm{H}) ; 13 \mathrm{C}$ NMR (126 MHz, $\left.\mathrm{CDCl}_{3}\right), \delta 141.4,137.1,134.0,134.0,132.6,130.8,128.5,128.5,128.0,127.7$, $126.6,126.4,125.5,125.4,78.2,77.9,65.2,64.3,61.0,60.5,59.2,58.2,42.1,40.8,31.7 ; 11 \mathrm{~B}$ NMR (160 MHz, CDCl $), \delta 27.04$; IR (film)vmax 3057, 3027, 2903, 1950, 1600, 1312, 1261; $\delta$; 
IR (film)vmax 3057, 3027, 2903, 1950, 1312, 1261; HRMS(EI) calculated. for $\mathrm{C}_{24} \mathrm{H}_{23} \mathrm{O}_{3} \mathrm{~B}$, $[\mathrm{M}+\mathrm{H}]+; 370.1740$, found 370.3520

\section{NOE Studies}

NOE studies were performed on (tertbutyl substrate with Boron) to confirm the trans stereochemistry of our two substituents. Table $\mathbf{X}$ shows the relative intensities of the peaks for hydrogens in question. From the below table, we can see that $\mathrm{H}_{7}$ sees $\mathrm{H} 8 \mathrm{~b}$ but cannot see $\mathrm{H} 8 \mathrm{a}$, and $\mathrm{H}_{6}$ can see $\mathrm{H}_{8}$ a but not $\mathrm{H}_{8 b}$. Although we have these results, the intensities do not exactly match expected ratios for a molecule in a chair conformation. However, through process of elimination we know that it cannot be any other arrangement. If $\mathrm{H}_{7}$ was equatorial, it would not be able to see $\mathrm{H}_{8 \mathrm{a}}$ or $\mathrm{H}_{8 \mathrm{~b}}$ so it must be axial. Similarly, if $\mathrm{H}_{6}$ was equatorial it would have to see $\mathrm{H} 8 \mathrm{~b}$ much more strongly than it currently does. The odd intensity ratios in our NOE experiments are due to the boron distorting the ring as it strains to move towards a $120^{\circ}$ bond angle. The $\mathrm{J}$ coupling values also help to prove this. 1,2 diaxial hydrogens ( $\mathrm{H}_{6}$ and $\mathrm{H}_{7}$, and $\mathrm{H}_{6}$ and $\mathrm{H}_{8 \mathrm{~b}}$ ) should have coupling values between $8-13 \mathrm{~Hz}$, but here $\mathrm{J}_{7,6}=7.0 \mathrm{~Hz}$ and $\mathrm{J}_{8 \mathrm{~b}, 6}=8.0 \mathrm{~Hz}$ which are a little low due to the distorted chair shape. Although the coupling between an axial and equatorial hydrogen in a 1,2 relationship is about right $\left(\mathrm{J}_{8 \mathrm{a}, 6}=3.7 \mathrm{~Hz}\right)$, the relationship between geminal hydrogens is also a little lower than what would be expected $\left(\mathrm{J}_{8 \mathrm{a}, 8 \mathrm{~b}}=11.5 \mathrm{~Hz}\right)$. We can further confirm the trans nature of our substrates by comparing the NOE studies of (tertbutyl substrate) with the crystal structure taken of (cinnamyl derivative) where the ring substituents come off trans and the bond angle of boron is $123.3^{\circ}$. Thus, we can infer that all of our substrates contain the same stereochemistry.

Table X. How strongly different hydrogens see each other in (tbu substrate) by NOE experiments.

\begin{tabular}{|l|l|l|l|l|l|l|}
\hline & $\mathrm{H}_{4}$ & $\mathrm{H}_{5}$ & $\mathrm{H}_{6}$ & $\mathrm{H}_{7}$ & $\mathrm{H}_{8 \mathrm{a}}$ & $\mathrm{H} 8 \mathrm{~b}$ \\
\hline $\mathrm{H}_{6}$ & $2.2 \%$ & $3.6 \%$ & - & $2.1 \%$ & $1.2 \%$ & no \\
\hline $\mathrm{H}_{7}$ & $0.6 \%$ & $2.5 \%$ & $1.4 \%$ & - & no & $1.5 \%$ \\
\hline $\mathrm{H}_{8 \mathrm{a}}$ & no & $4 \%$ & no & no & - & $20 \%$ \\
\hline $\mathrm{H}_{8 \mathrm{~b}}$ & $0.7 \%$ & $3.0 \%$ & $0.4 \%$ & $2.4 \%$ & $19 \%$ & - \\
\hline
\end{tabular}

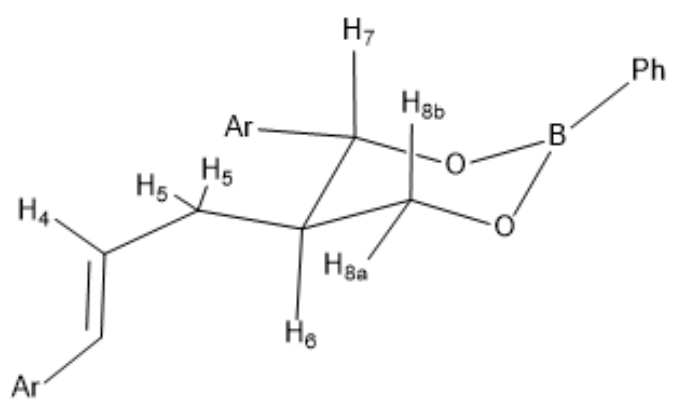




\section{NMR Data}

2a. 5-cinnamyl-2,4-diphenyl-1,3,2-dioxaborinane $1 \mathrm{H}$ NMR
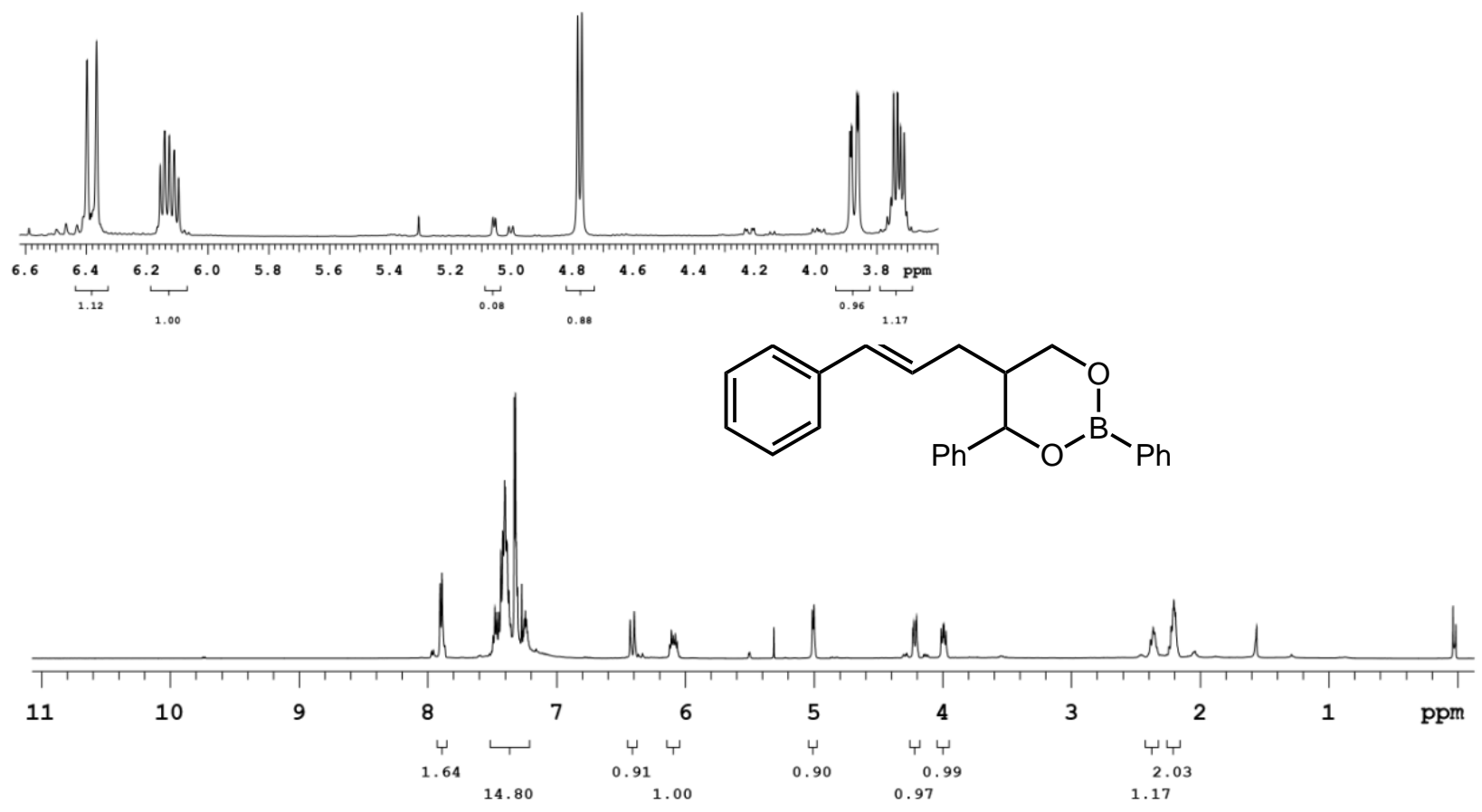

2a. 5-cinnamyl-2,4-diphenyl-1,3,2-dioxaborinane $13 \mathrm{C}$ NMR

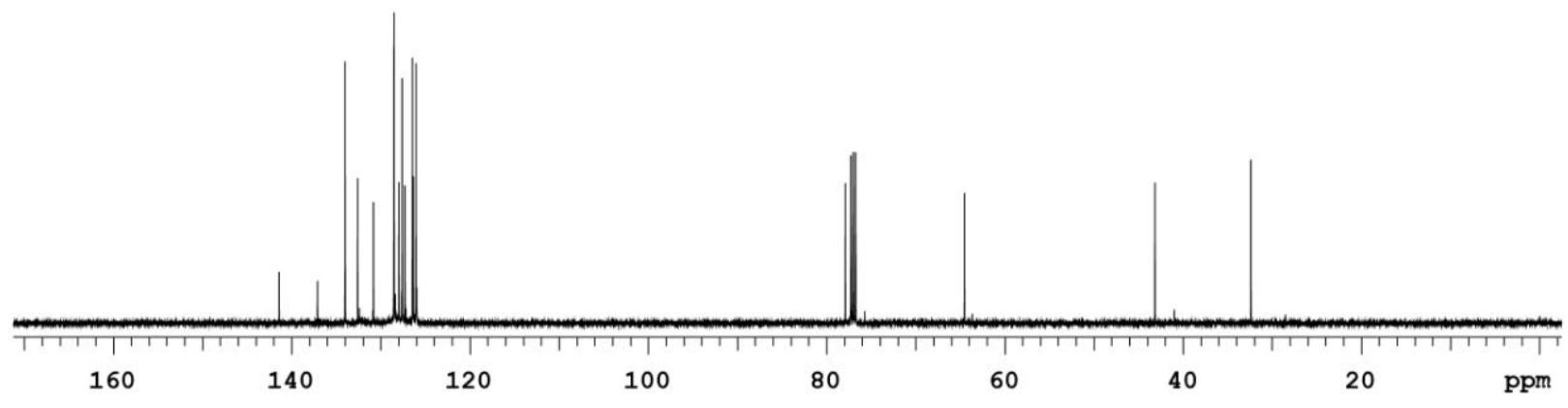


2b. (E)-4-(4-bromophenyl)-5-(3-(4-bromophenyl)allyl)-2-phenyl-1,3,2-dioxaborinane 1 H NMR

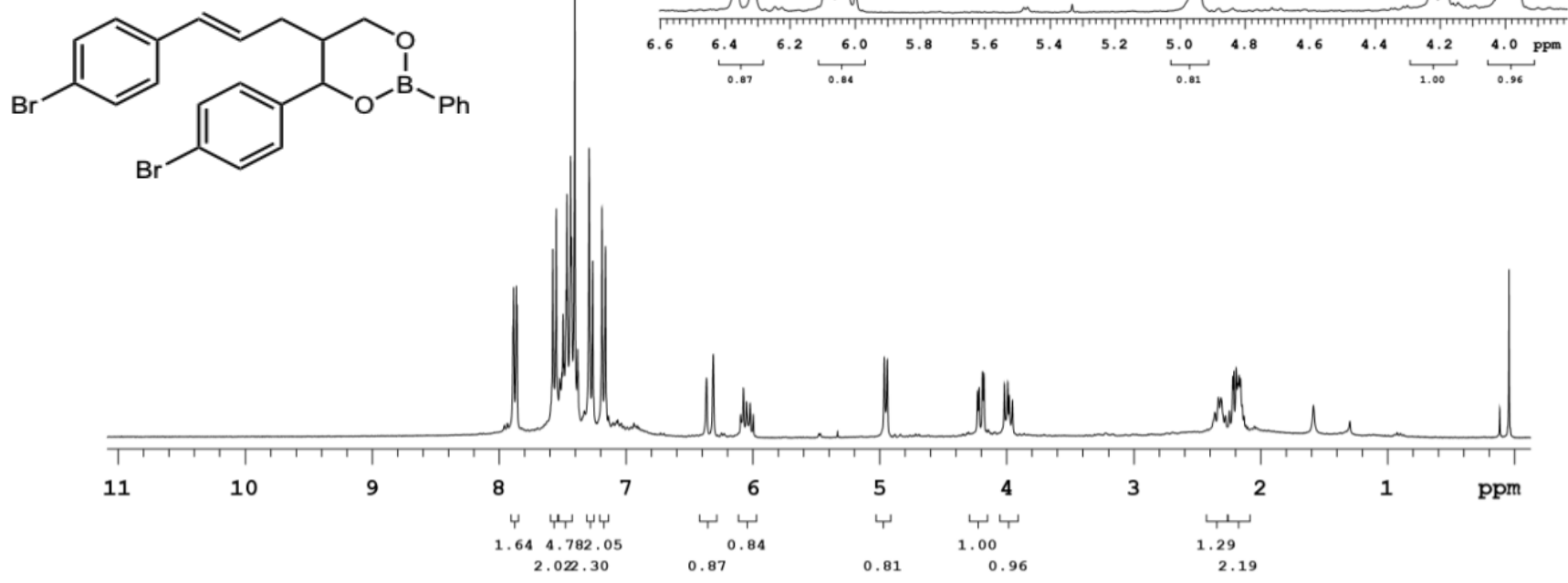

2b. (E)-4-(4-bromophenyl)-5-(3-(4-bromophenyl)allyl)-2-phenyl-1,3,2-dioxaborinane 13C NMR

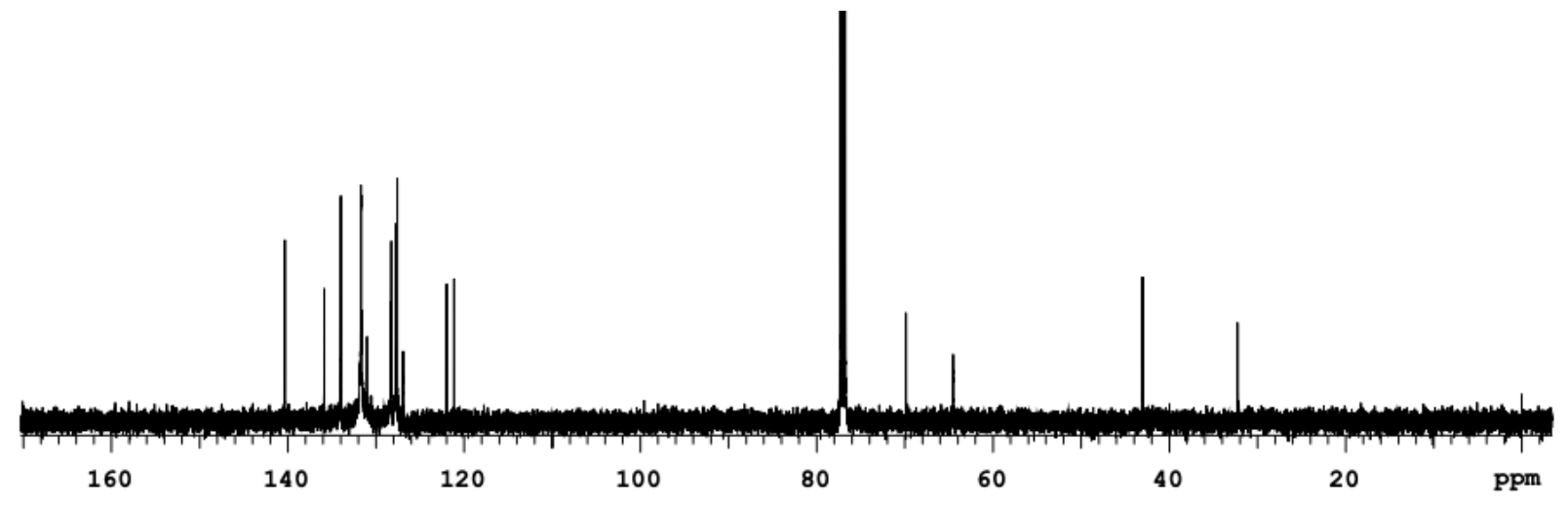


2c. (E)-4-(4-methoxyphenyl)-5-(3-(4-methoxyphenyl)allyl)-2-phenyl-1,3,2-dioxaborinane $1 \mathrm{H}$ NMR
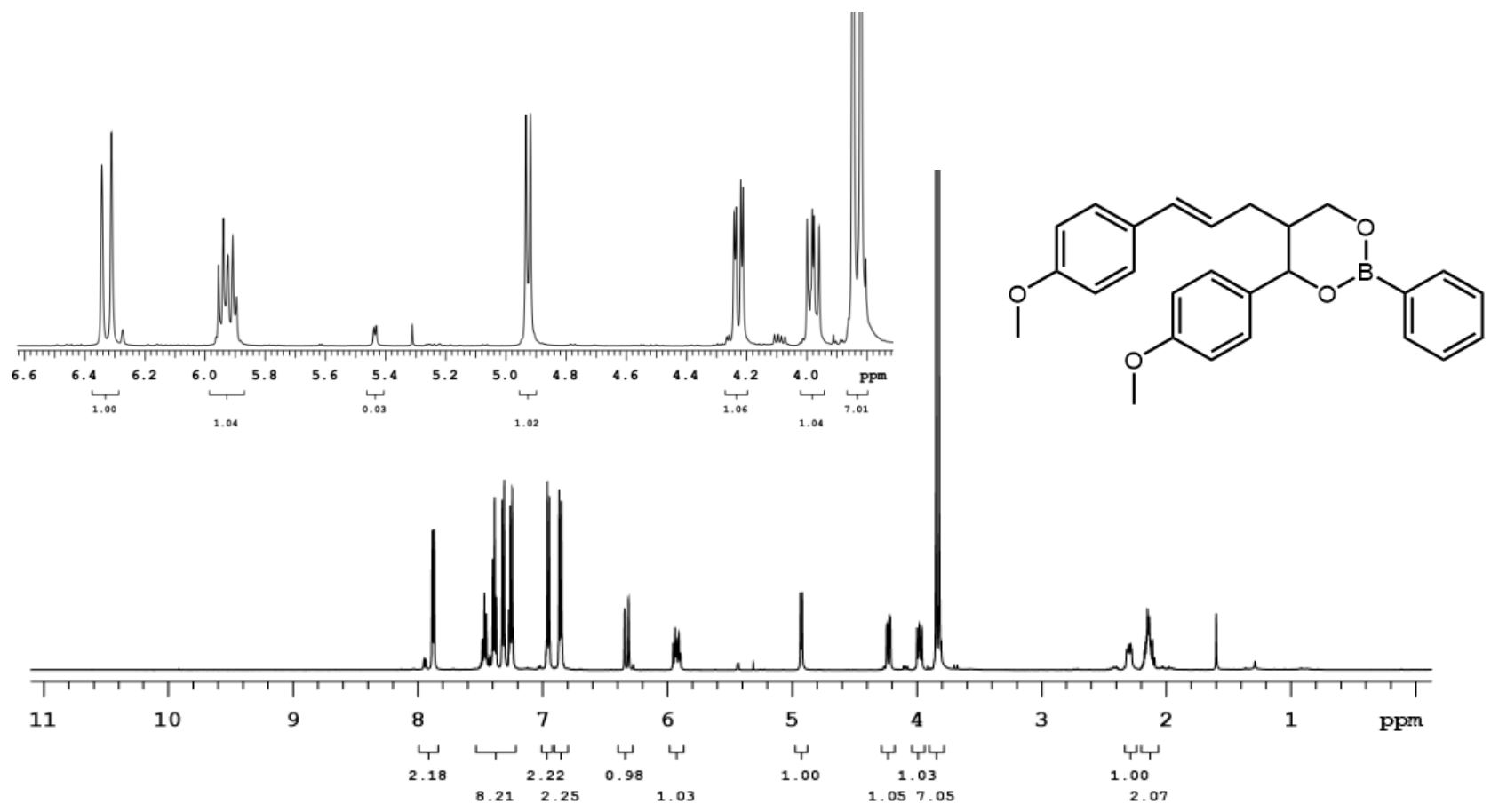

2c. (E)-4-(4-methoxyphenyl)-5-(3-(4-methoxyphenyl)allyl)-2-phenyl-1,3,2-dioxaborinane ${ }_{13 \mathrm{C}}$ NMR

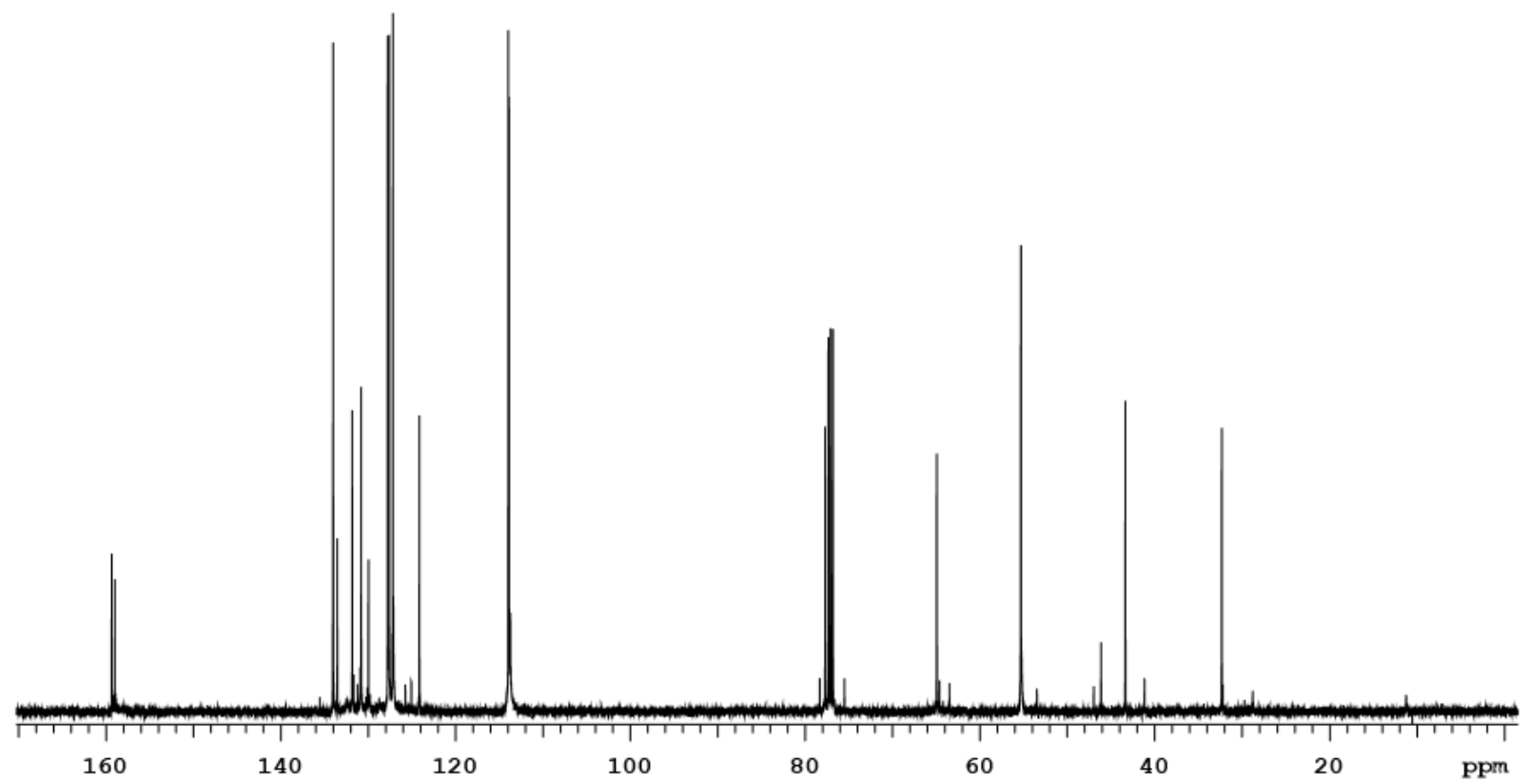


2d. (E)-2-phenyl-4-(p-tolyl)-5-(3-(p-tolyl)allyl)-1,3,2-dioxaborinane $1 \mathrm{H}$ NMR
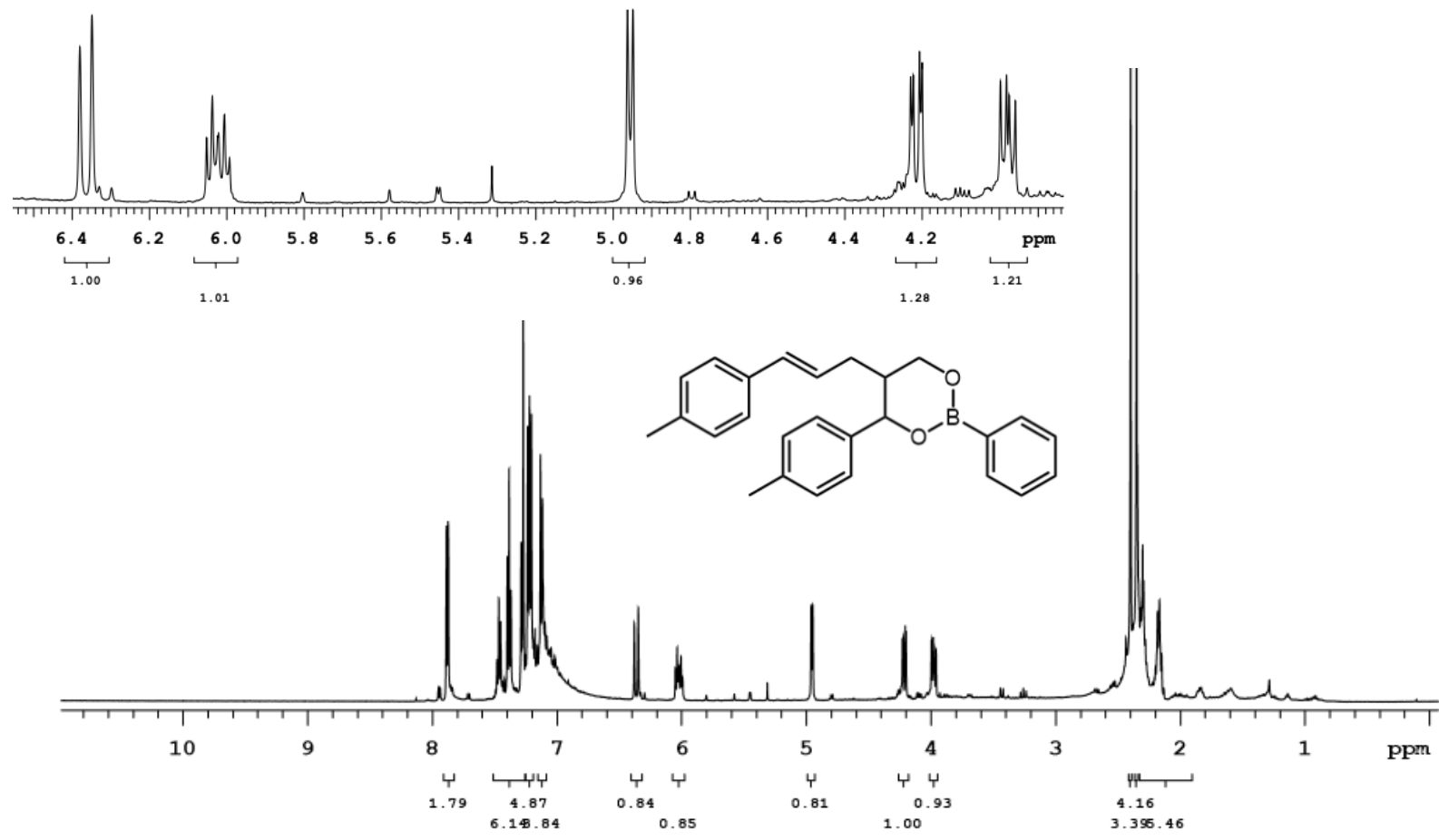

2d. (E)-2-phenyl-4-(p-tolyl)-5-(3-(p-tolyl)allyl)-1,3,2-dioxaborinane ${ }_{13} \mathrm{C}$ NMR

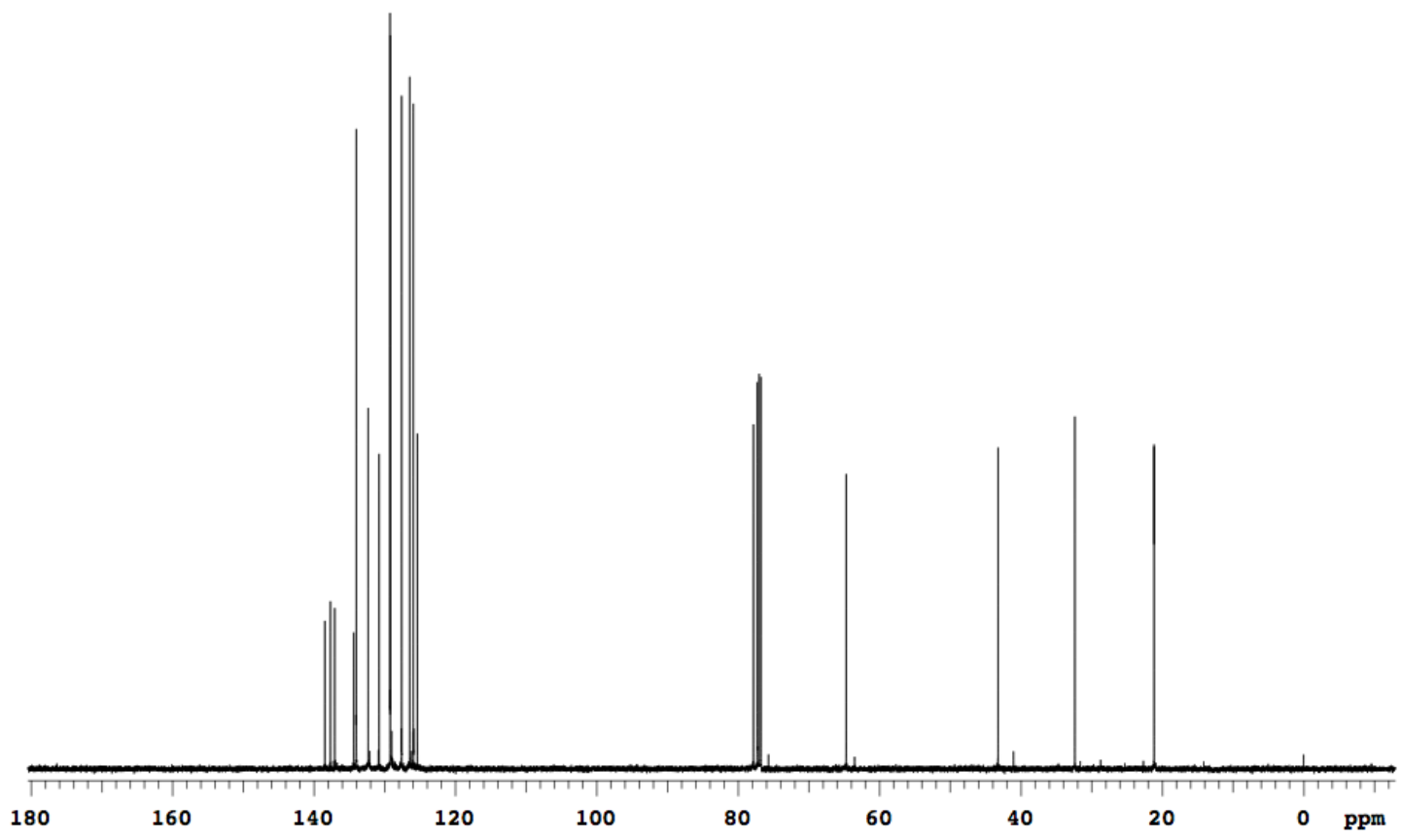




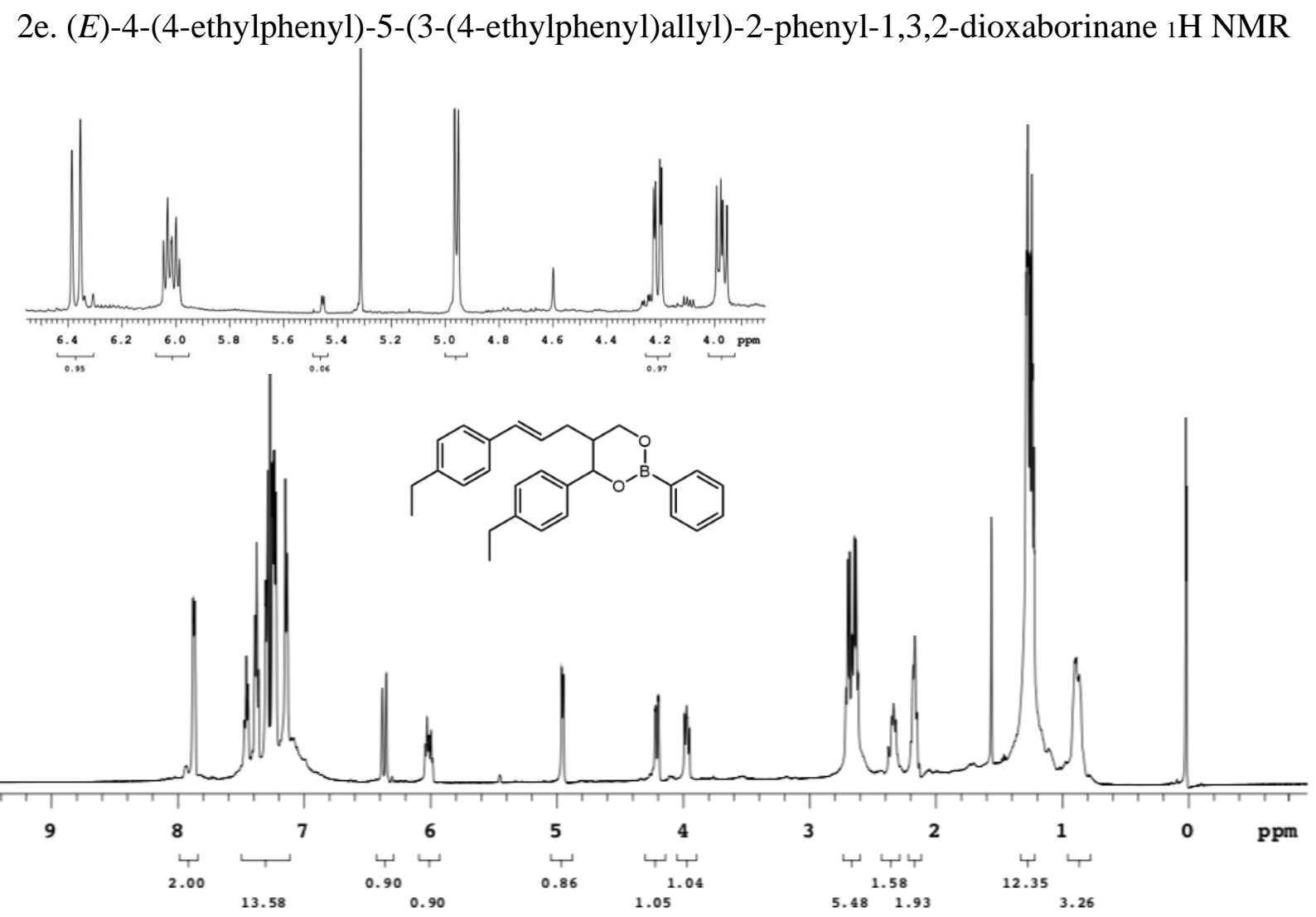

2e. (E)-4-(4-ethylphenyl)-5-(3-(4-ethylphenyl)allyl)-2-phenyl-1,3,2-dioxaborinane ${ }_{13} \mathrm{C}$ NMR

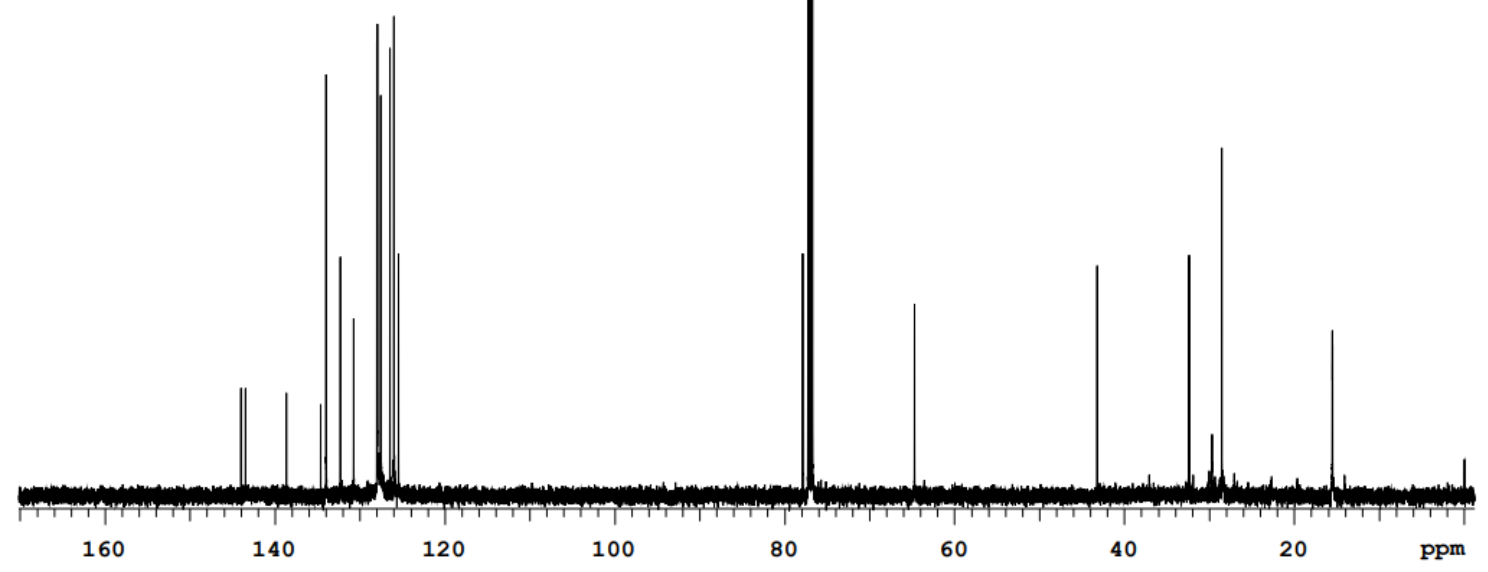


2f. (E)-4-(4-pentylphenyl)-5-(3-(4-pentylphenyl)allyl)-2-phenyl-1,3,2-dioxaborinane $1 \mathrm{H}$ NMR
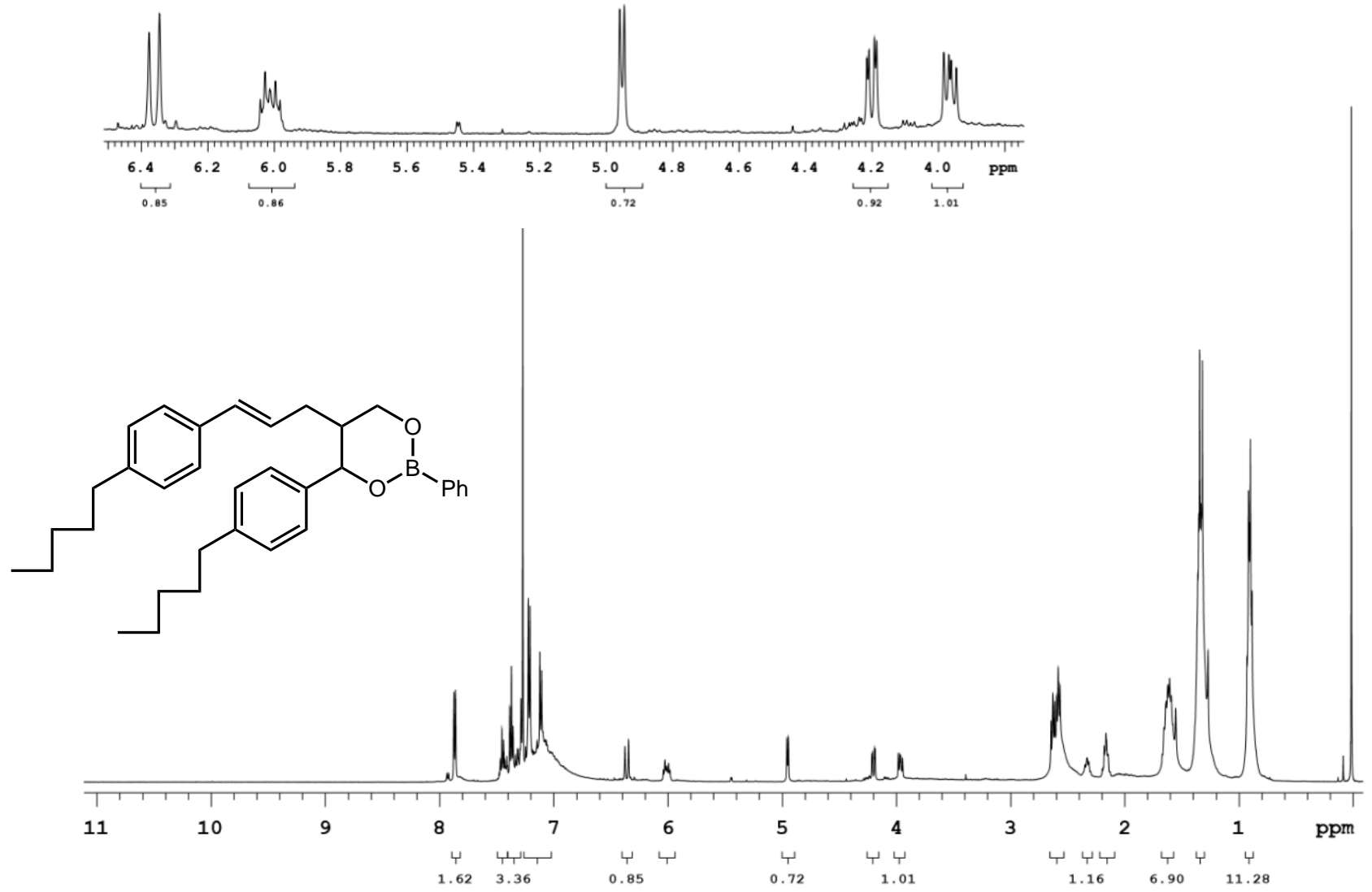

2f. (E)-4-(4-pentylphenyl)-5-(3-(4-pentylphenyl)allyl)-2-phenyl-1,3,2-dioxaborinane 13C NMR

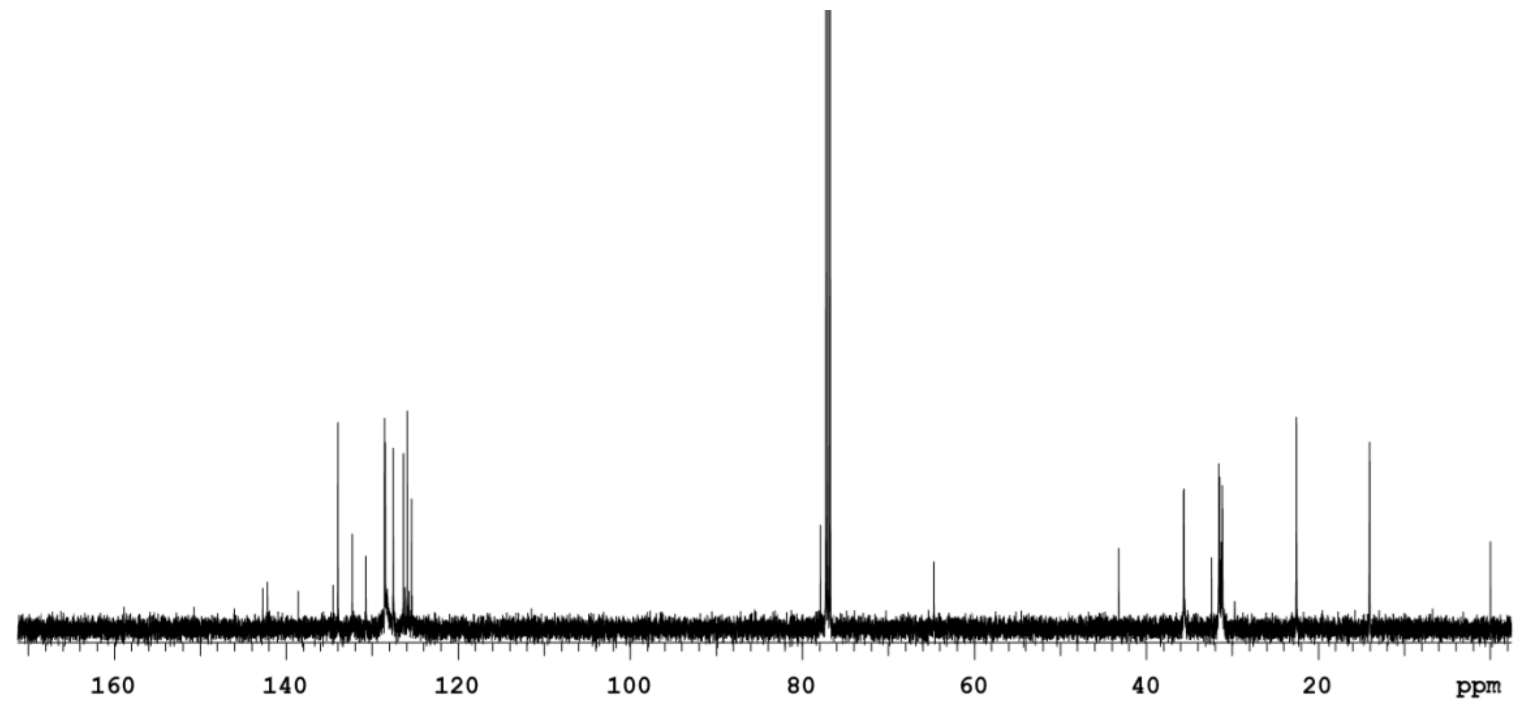


2g. (E)-4-(4-(tert-butyl)phenyl)-5-(3-(4-(tert-butyl)phenyl)allyl)-2-phenyl-1,3,2-dioxaborinane ${ }_{1} \mathrm{H}$ NMR
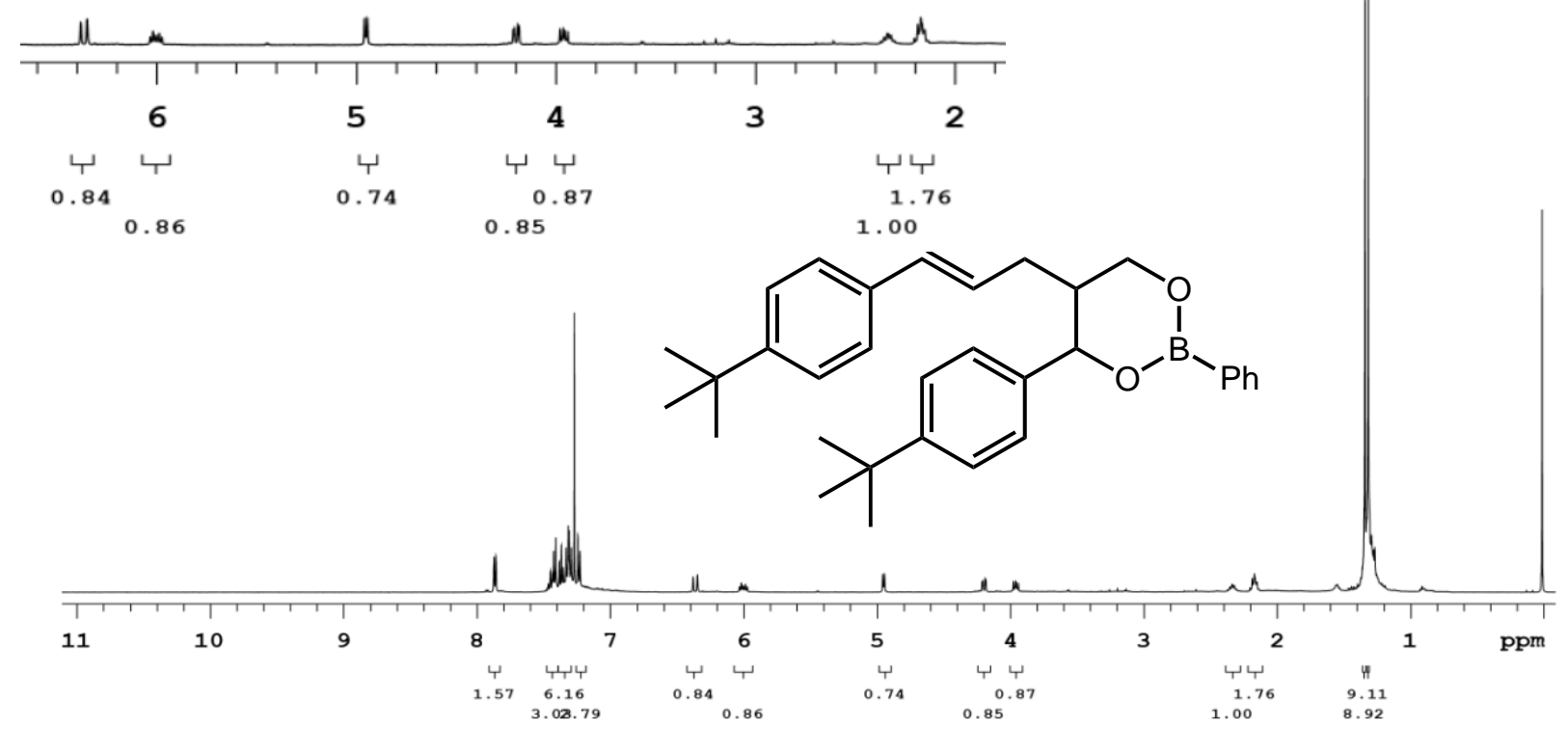

2g. (E)-4-(4-(tert-butyl)phenyl)-5-(3-(4-(tert-butyl)phenyl)allyl)-2-phenyl-1,3,2-dioxaborinane ${ }_{13} \mathrm{C}$ NMR

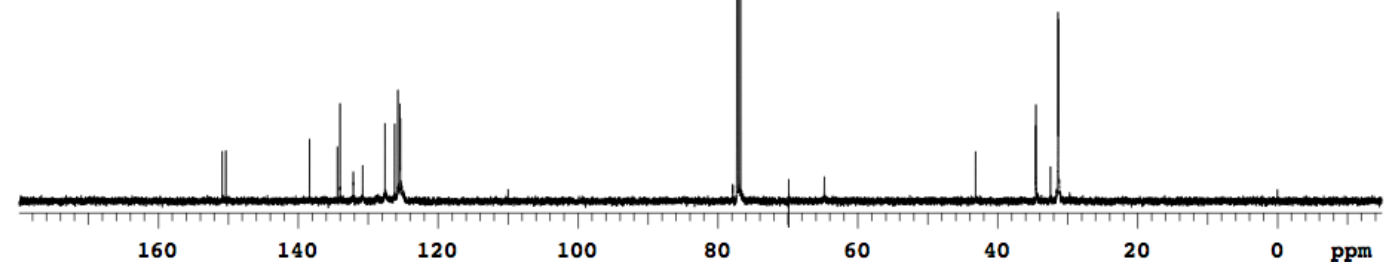


2h (E)-4-(4-chlorophenyl)-5-(3-(4-chlorophenyl)allyl)-2-phenyl-1,3,2-dioxaborinane $1 \mathrm{H}$ NMR

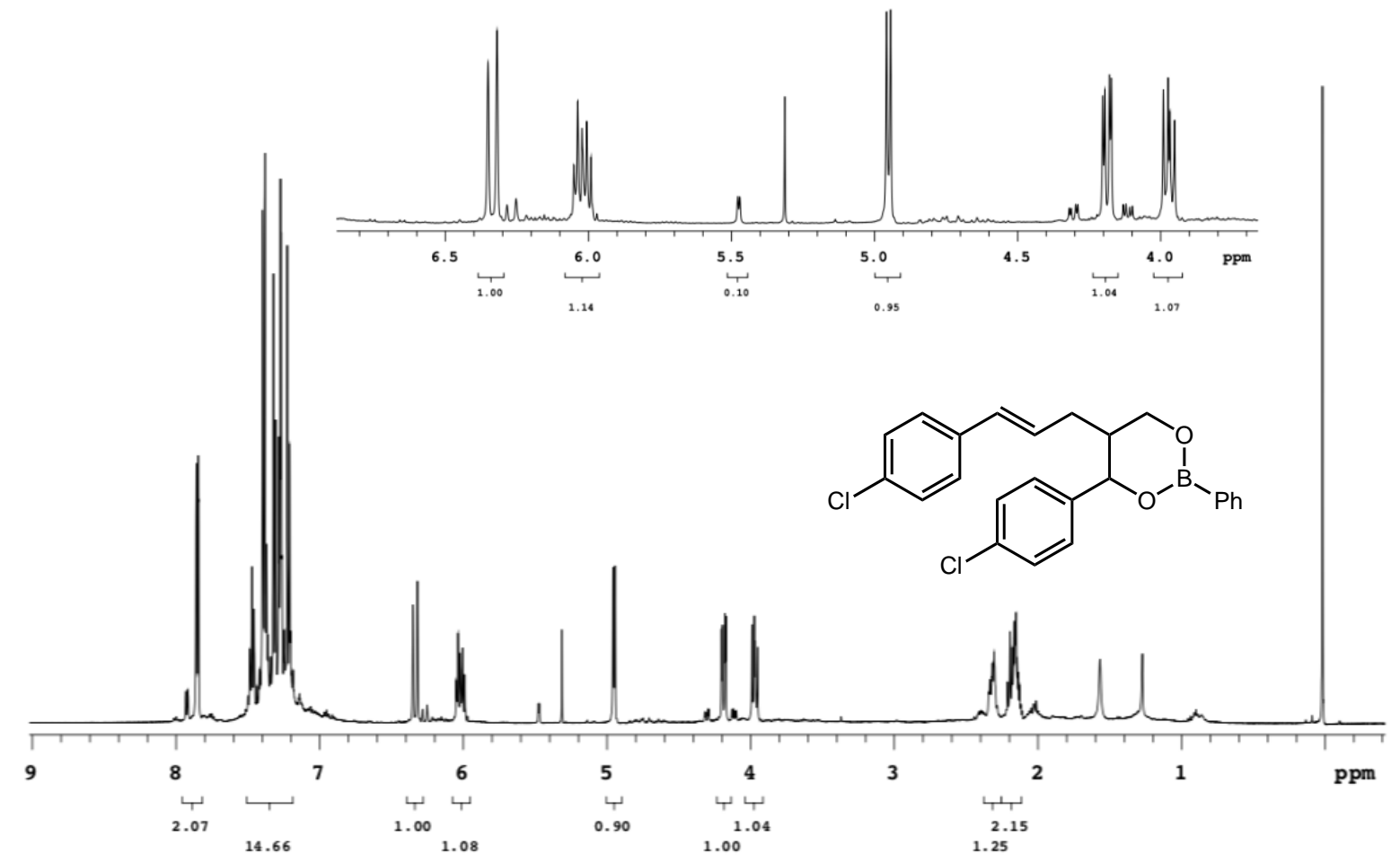

2h (E)-4-(4-chlorophenyl)-5-(3-(4-chlorophenyl)allyl)-2-phenyl-1,3,2-dioxaborinane ${ }_{13} \mathrm{C}$ NMR

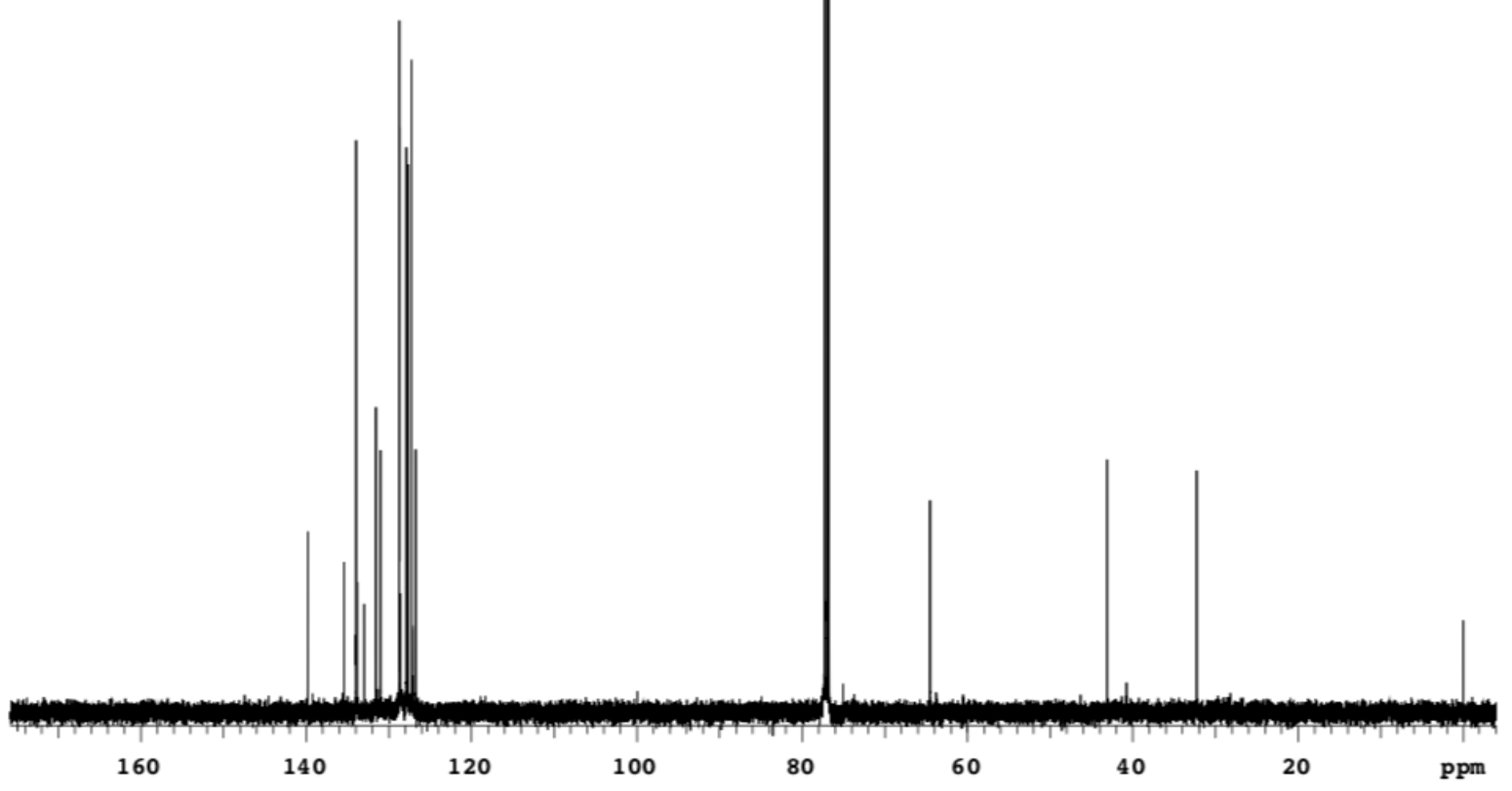


2i. (E)-2-phenyl-4-(4-(trifluoromethyl)phenyl)-5-(3-(4-(trifluoromethyl)phenyl)allyl)-1,3,2dioxaborinane ${ }_{1} \mathrm{H}$ NMR

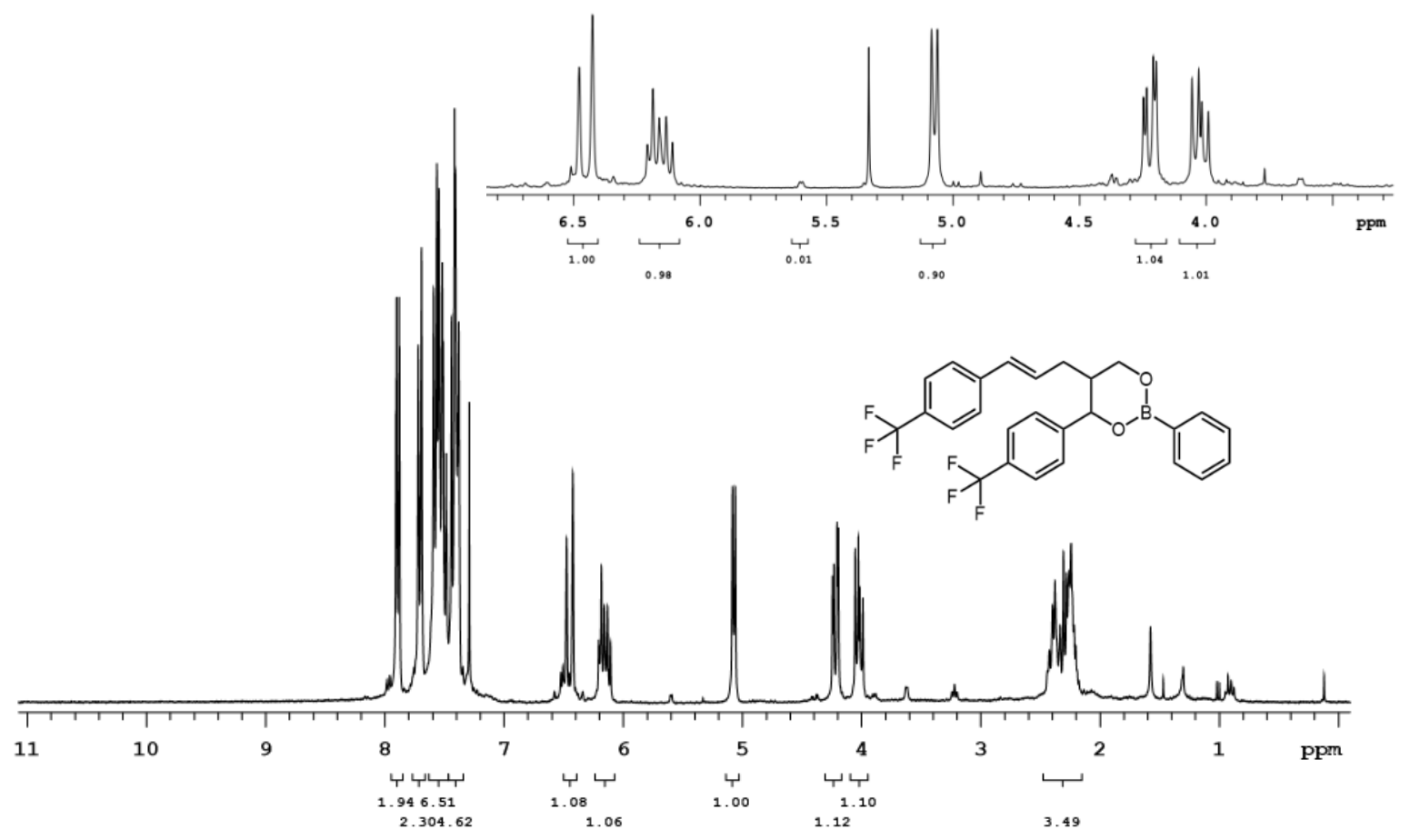

2i. (E)-2-phenyl-4-(4-(trifluoromethyl)phenyl)-5-(3-(4-(trifluoromethyl)phenyl)allyl)-1,3,2dioxaborinane ${ }_{13} \mathrm{C}$ NMR

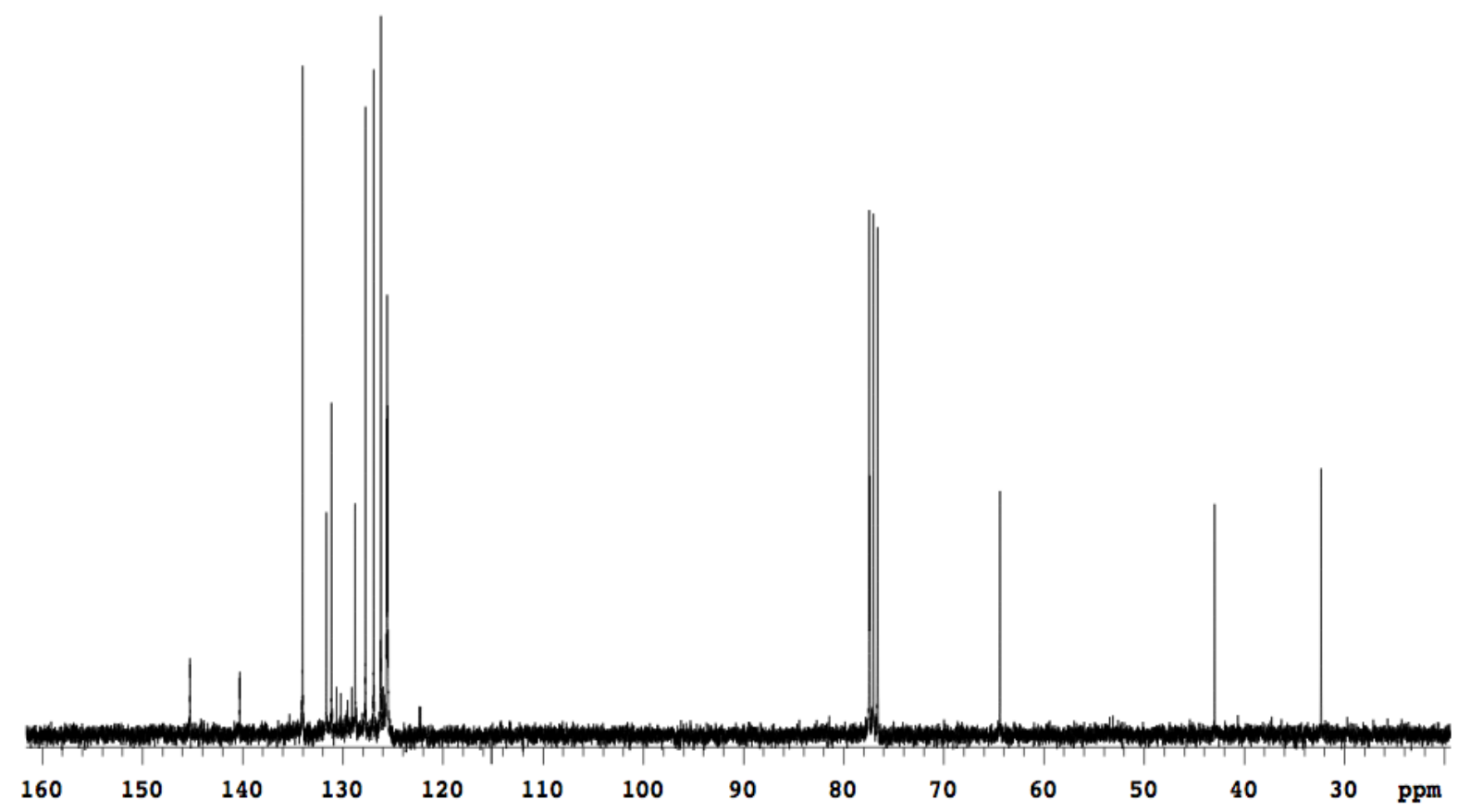


2j. (E)-2-phenyl-4-( $m$-tolyl)-5-(3-( $m$-tolyl)allyl)-1,3,2-dioxaborinane $1 \mathrm{H}$ NMR
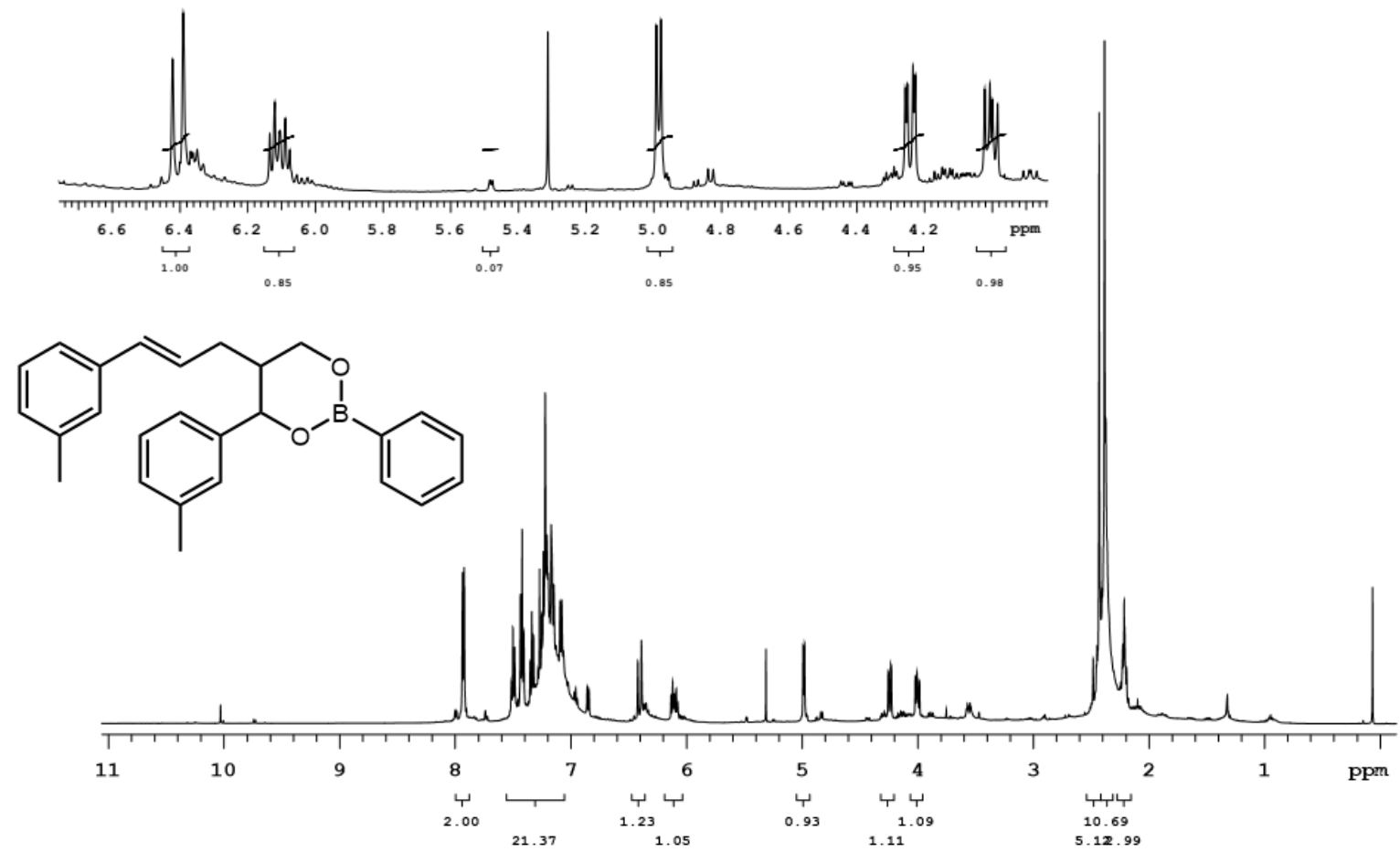

2j. (E)-2-phenyl-4-( $m$-tolyl)-5-(3-( $m$-tolyl)allyl)-1,3,2-dioxaborinane 13C NMR

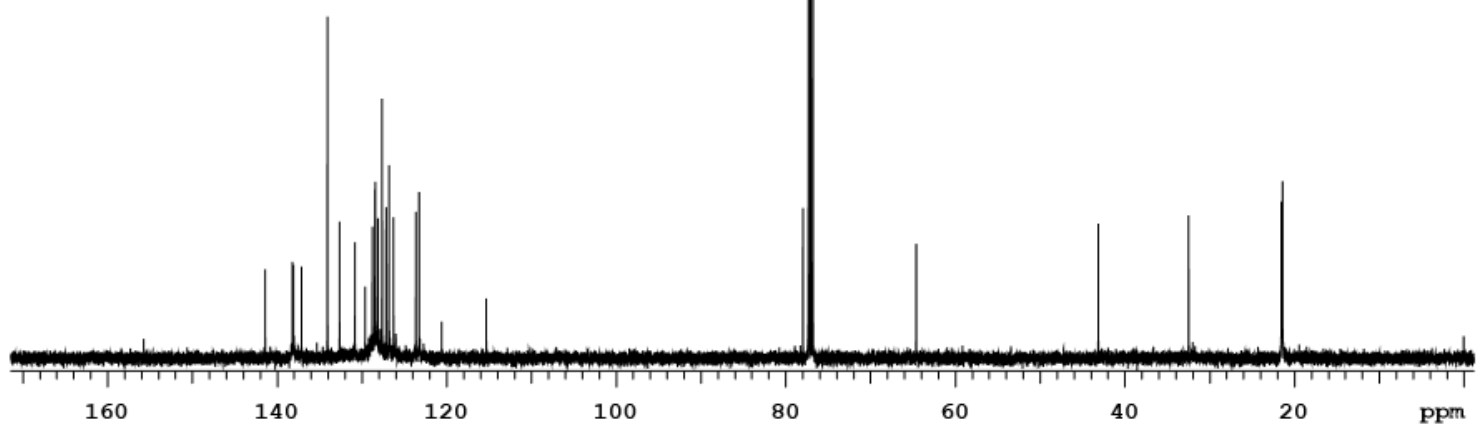


2k. (E)-4-(3-chlorophenyl)-5-(3-(3-chlorophenyl)allyl)-2-phenyl-1,3,2-dioxaborinane 1H NMR

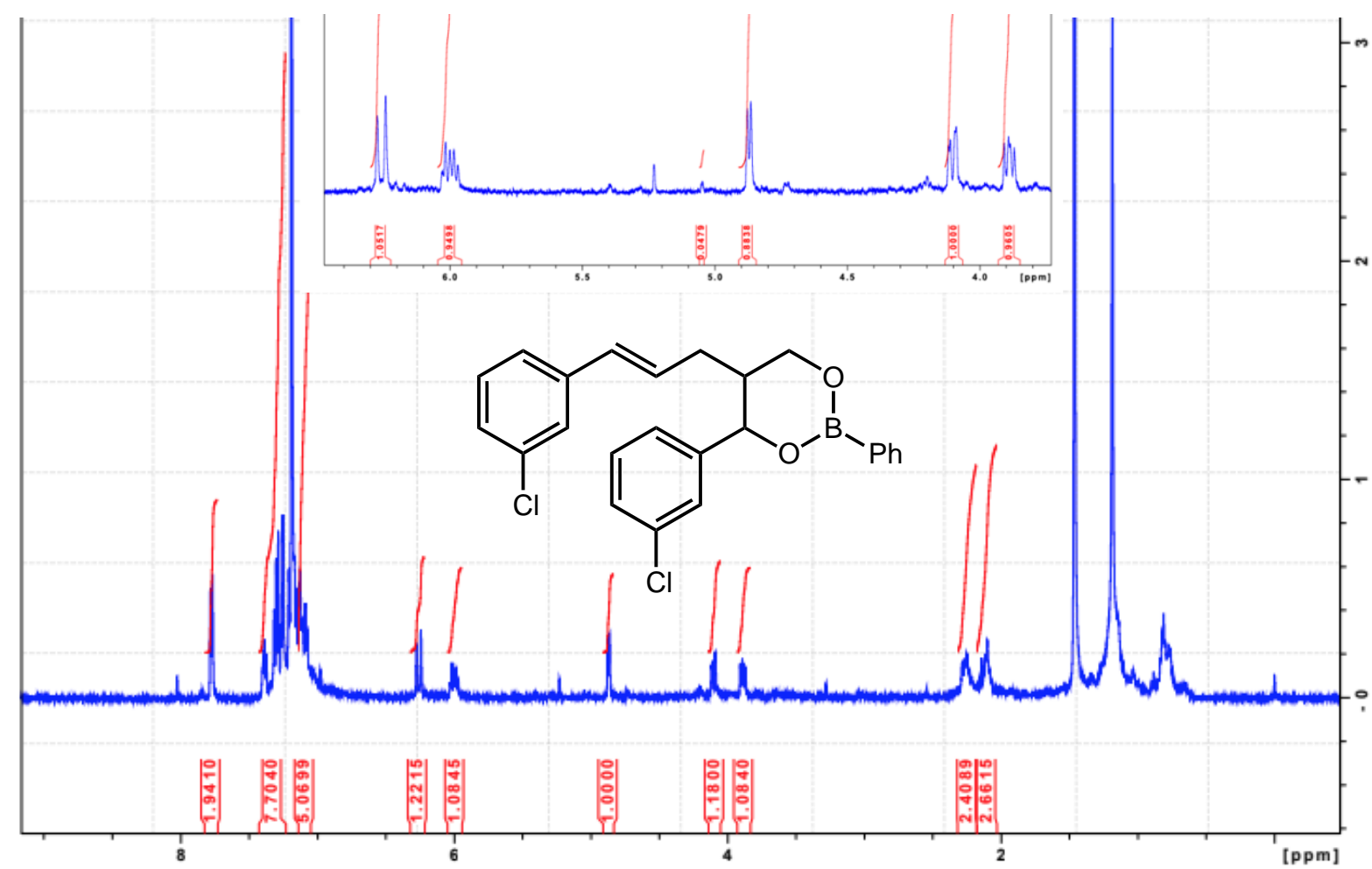

2k. (E)-4-(3-chlorophenyl)-5-(3-(3-chlorophenyl)allyl)-2-phenyl-1,3,2-dioxaborinane 13C NMR

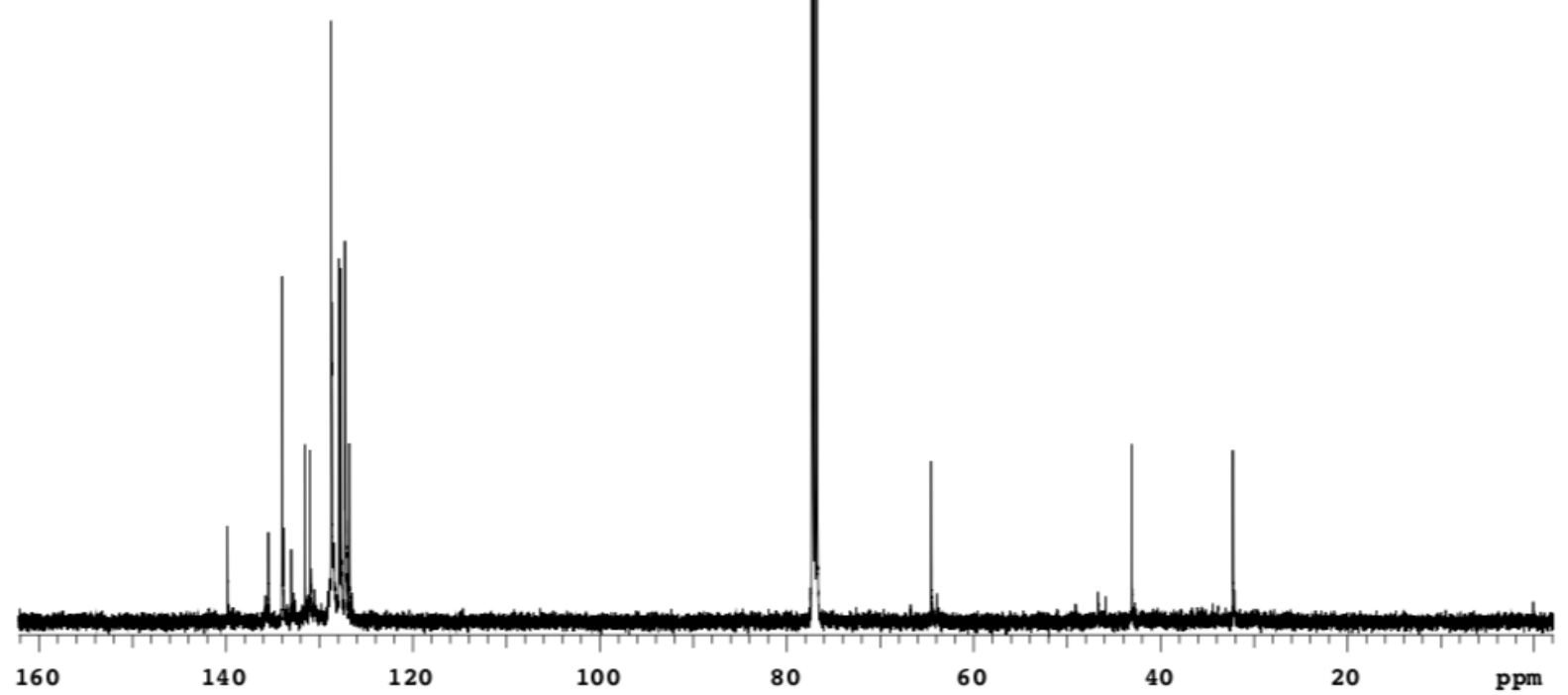


21. (E)-2-phenyl-4-(o-tolyl)-5-(3-(o-tolyl)allyl)-1,3,2-dioxaborinane $1 \mathrm{H}$ NMR
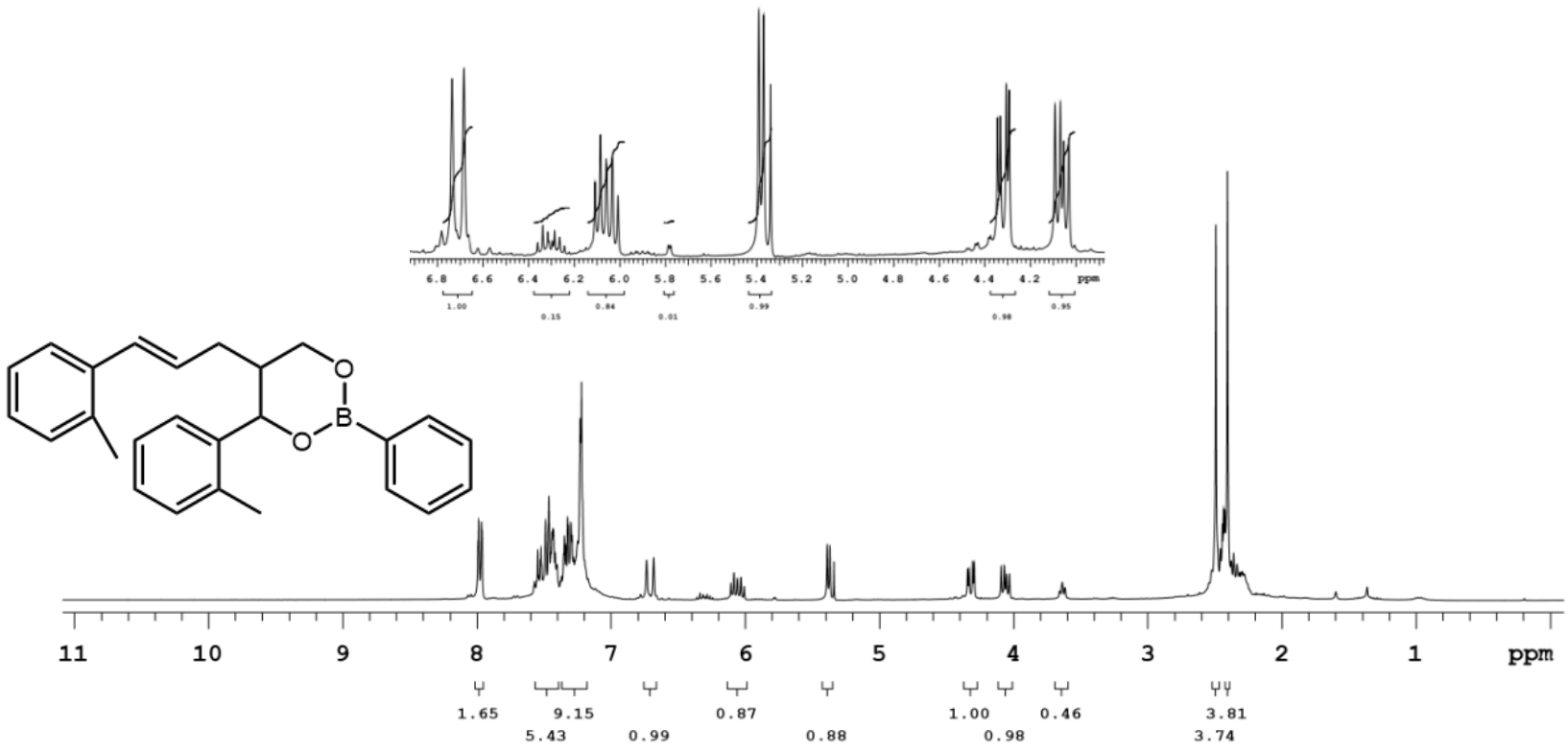

21. (E)-2-phenyl-4-(o-tolyl)-5-(3-(o-tolyl)allyl)-1,3,2-dioxaborinane $13 \mathrm{C}$ NMR

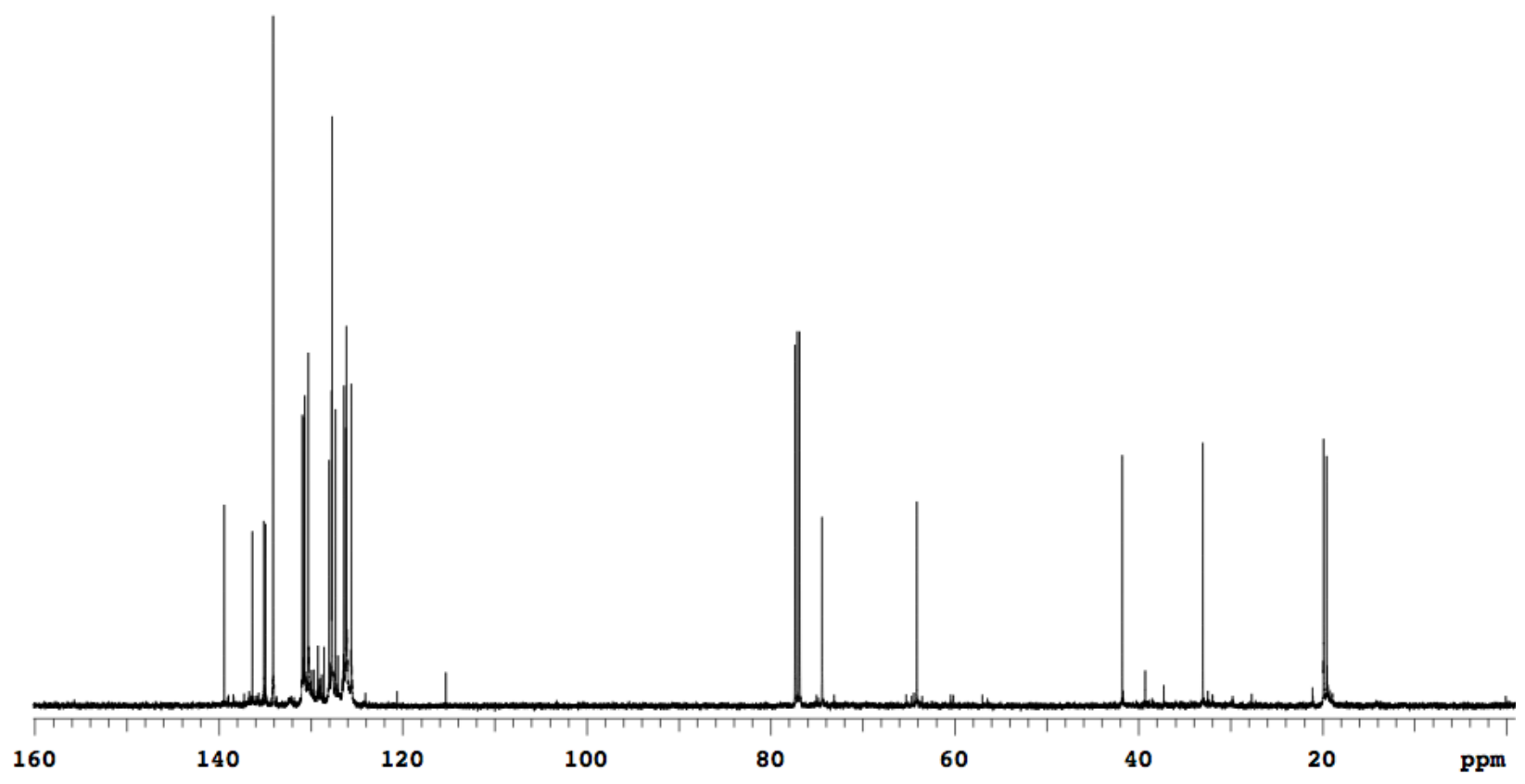


2m. (E)-4-(2-methoxyphenyl)-5-(3-(2-methoxyphenyl)allyl)-2-phenyl-1,3,2-dioxaborinane $1 \mathrm{H}$ NMR
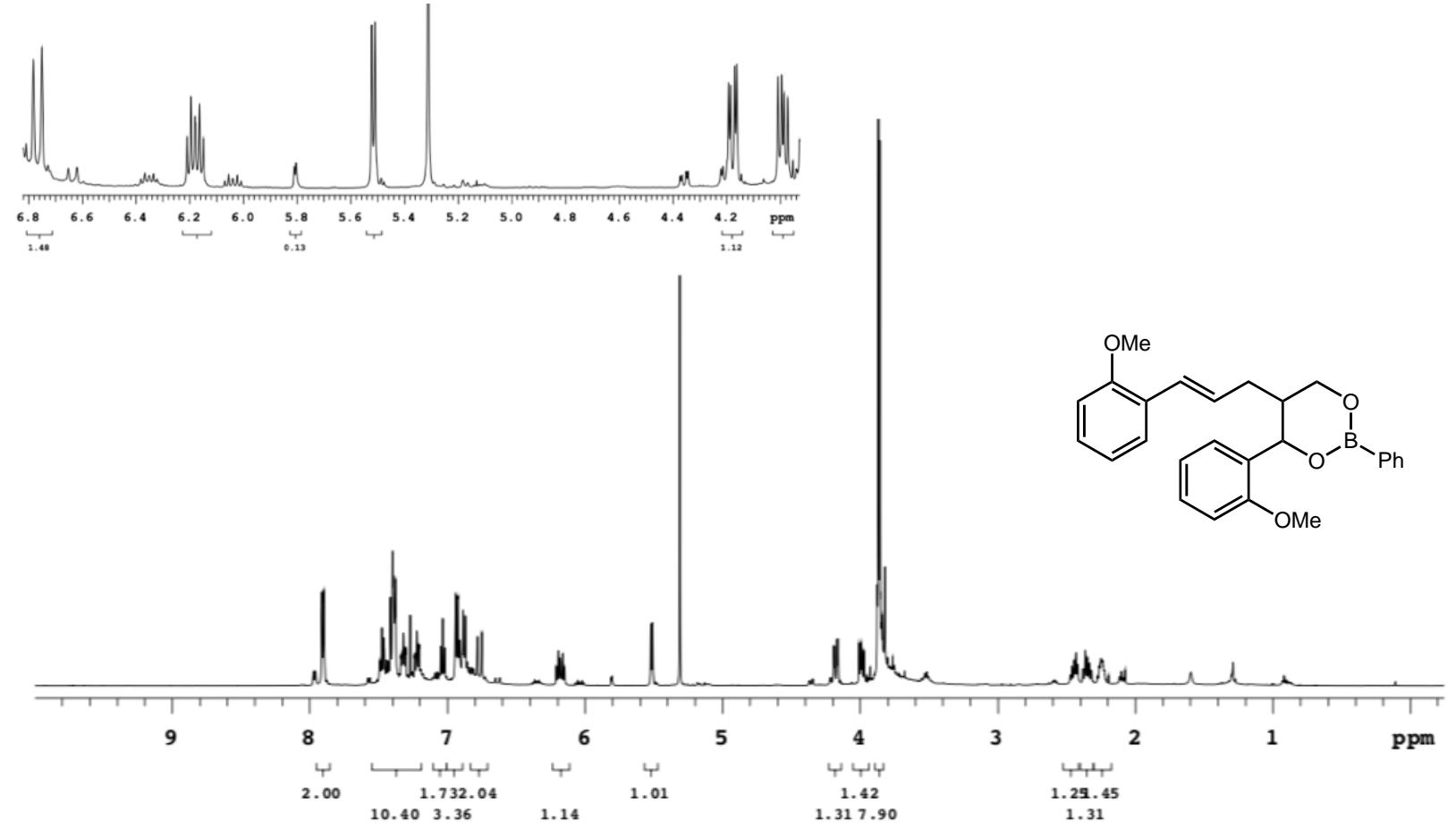

2m. (E)-4-(2-methoxyphenyl)-5-(3-(2-methoxyphenyl)allyl)-2-phenyl-1,3,2-dioxaborinane ${ }_{13} \mathrm{C}$ NMR

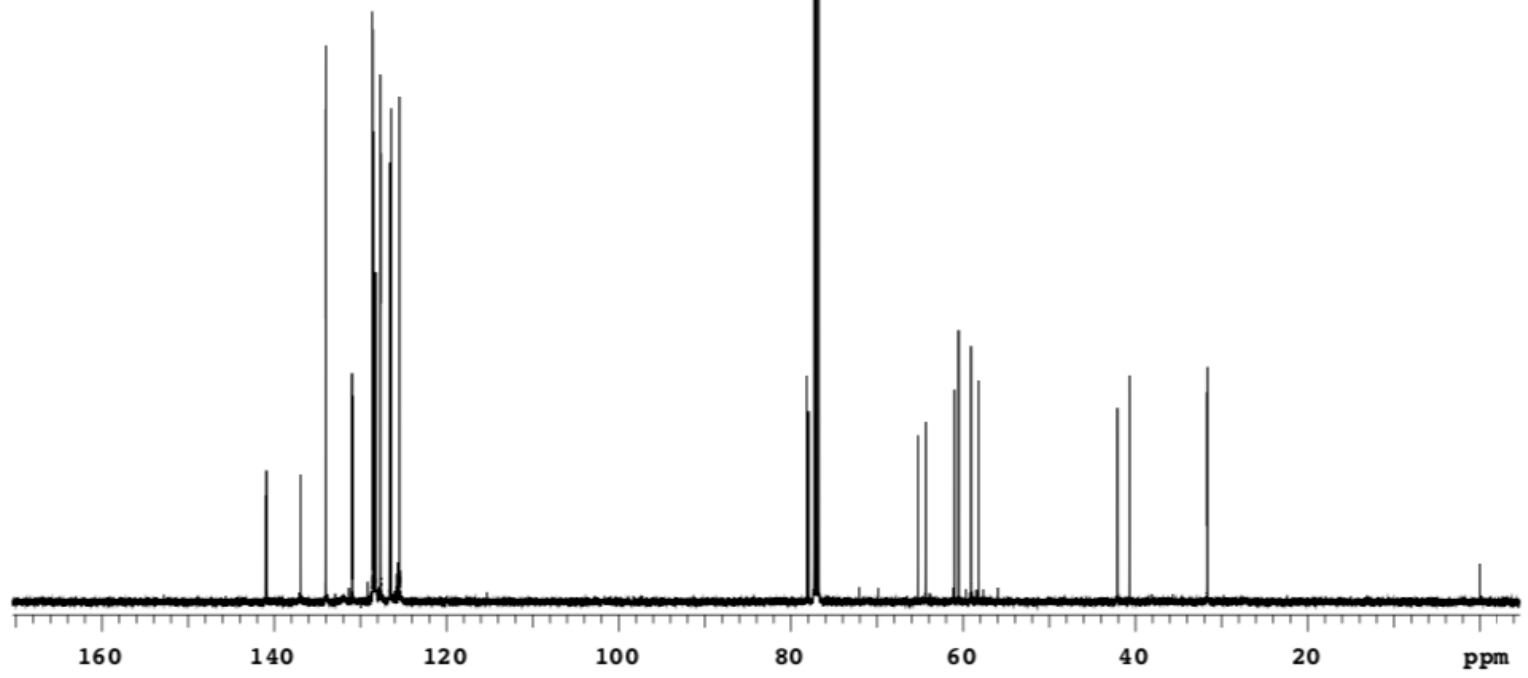


2n. (E)-1-(naphthalen-1-yl)-2-(3-(naphthalen-1-yl)allyl)propane-1,3-diol 1 H NMR

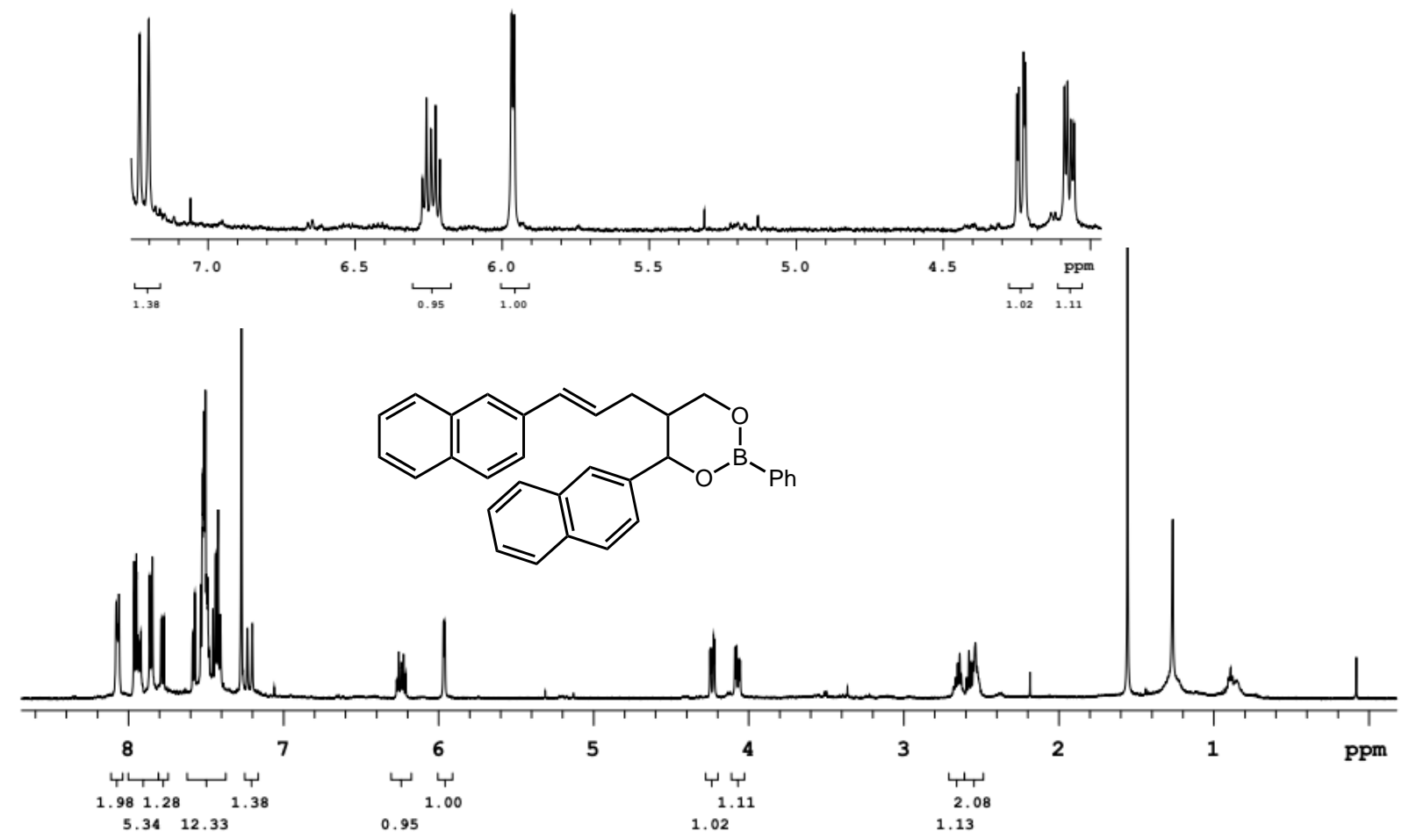

2n. (E)-1-(naphthalen-1-yl)-2-(3-(naphthalen-1-yl)allyl)propane-1,3-diol ${ }_{13} \mathrm{C}$ NMR

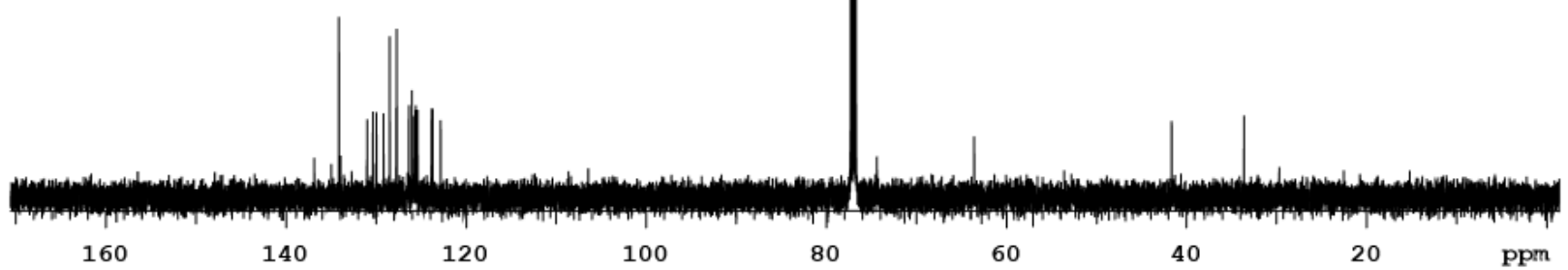


2o. (E)-4-(4-(tert-butyl)phenyl)-5-(3-(4-(tert-butyl)phenyl)allyl)-2-phenyl-1,3,2-dioxaborinane ${ }_{1} \mathrm{H}$ NMR

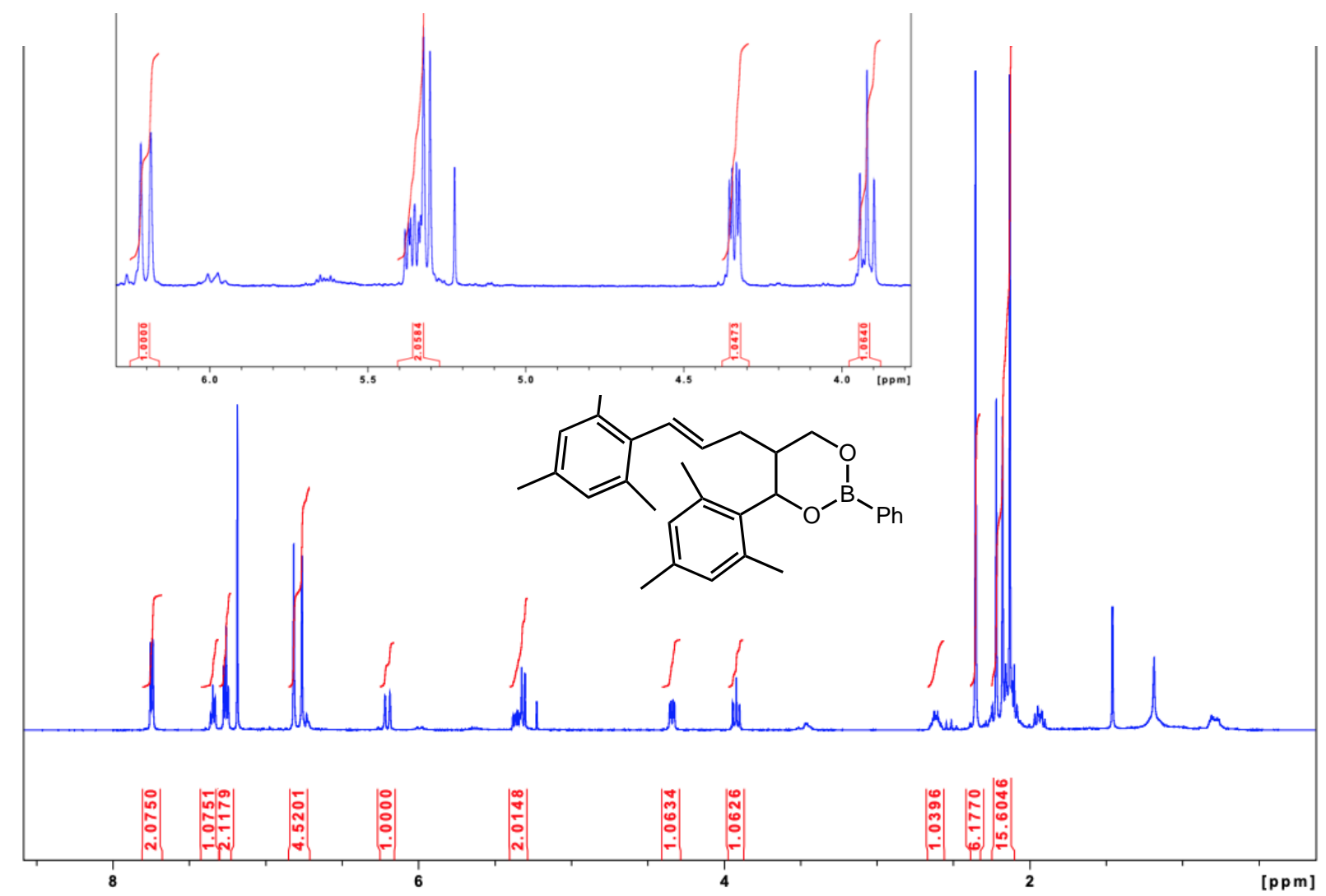

2o. (E)-4-(4-(tert-butyl)phenyl)-5-(3-(4-(tert-butyl)phenyl)allyl)-2-phenyl-1,3,2-dioxaborinane ${ }_{13} \mathrm{C}$ NMR

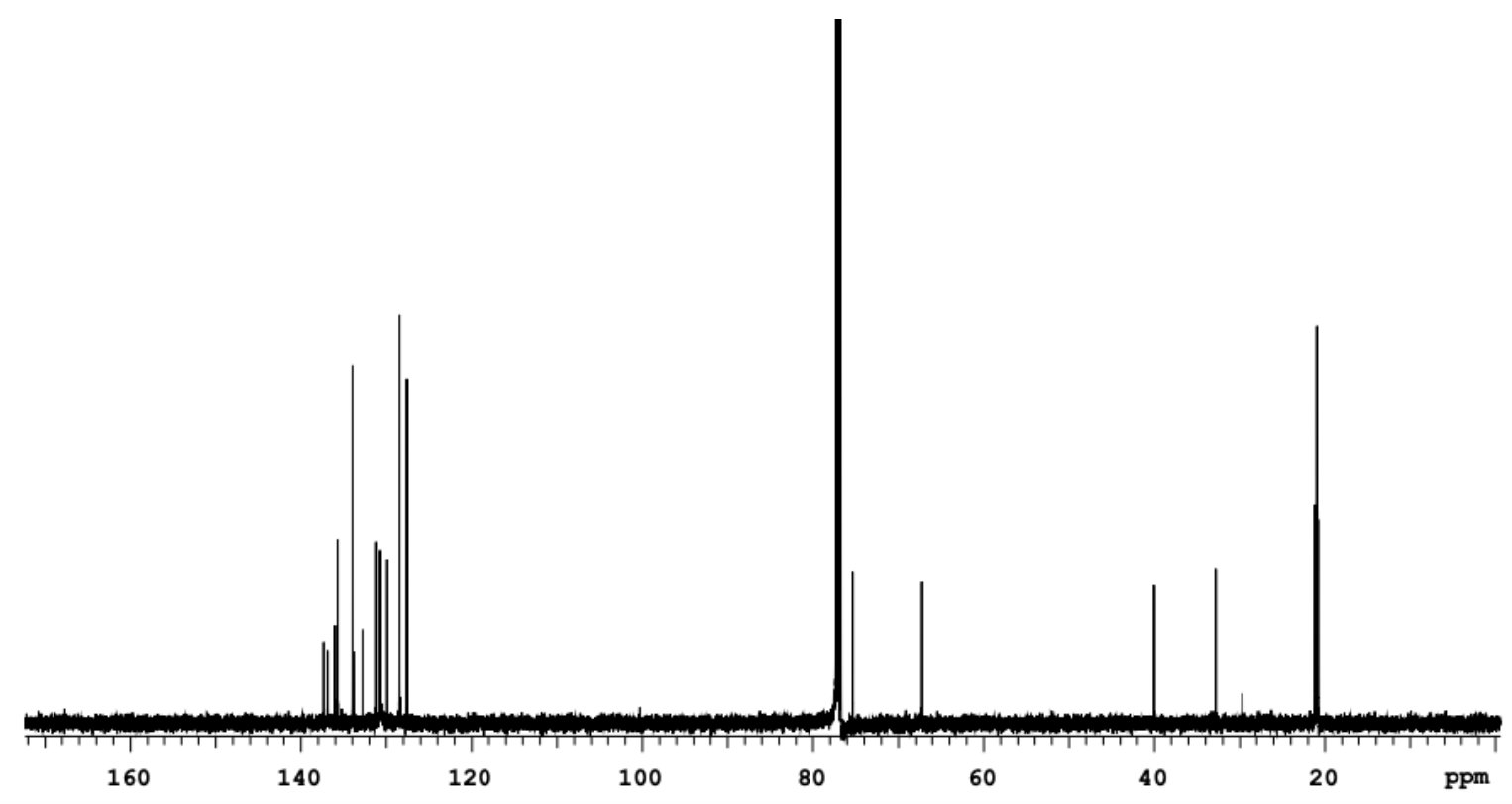


2p. (E)-3-phenyl-2-(3-phenylbut-2-en-1-yl)butane-1,3-diol 1H NMR

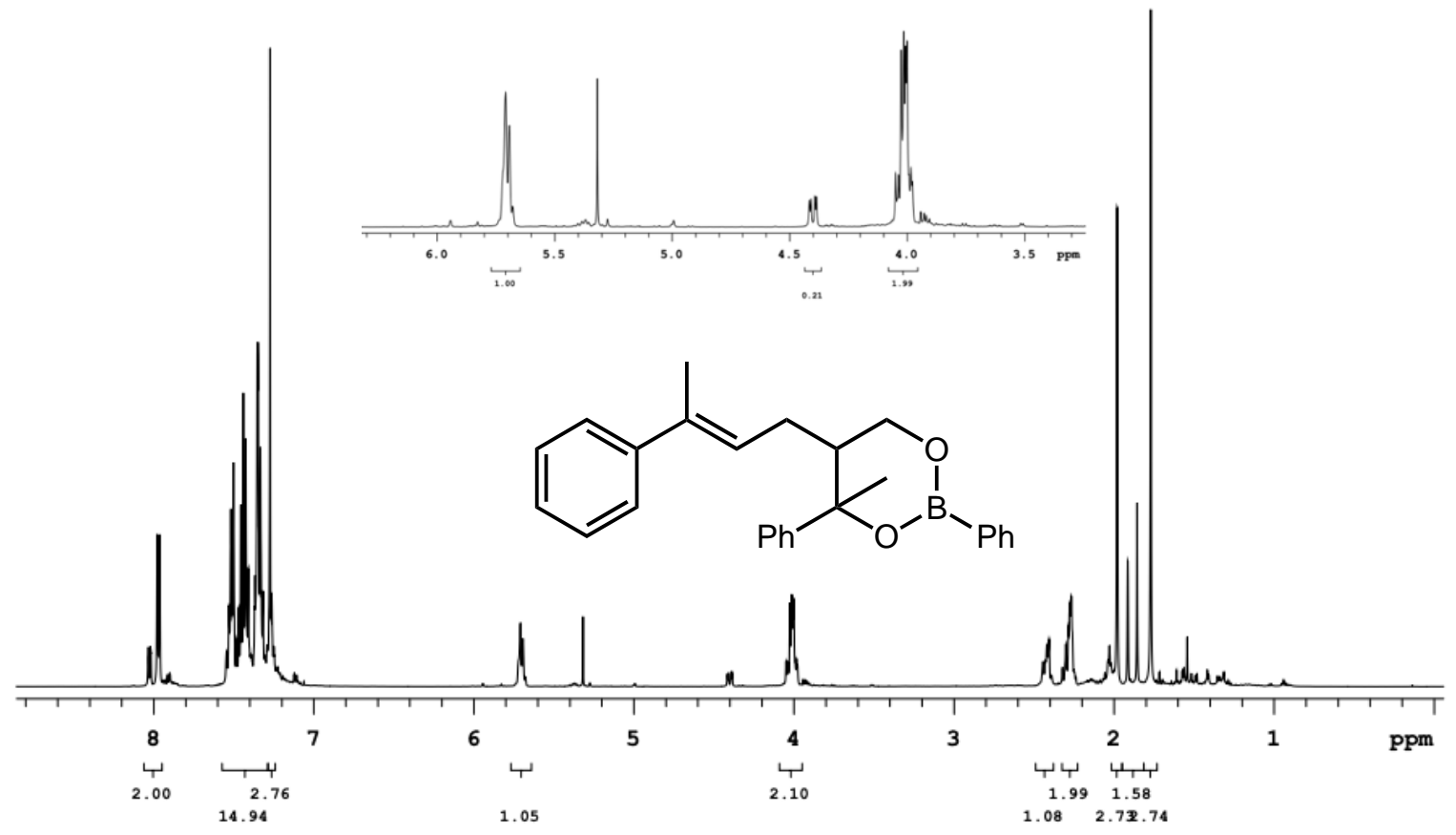

2p. (E)-3-phenyl-2-(3-phenylbut-2-en-1-yl)butane-1,3-diol 13C NMR

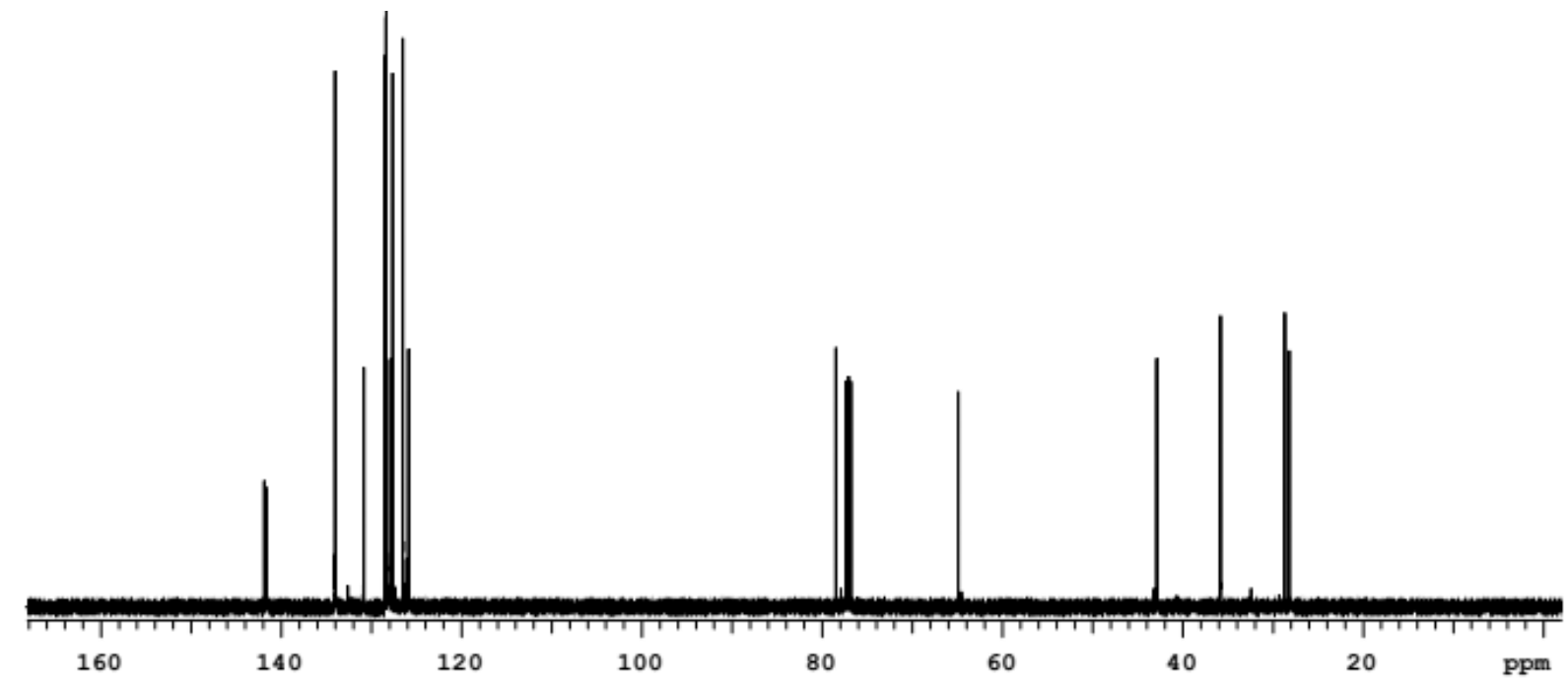


2q. (E)-4-(3,4-difluorophenyl)-5-(3-(3,4-difluorophenyl)allyl)-2-phenyl-1,3,2-dioxaborinane $1 \mathrm{H}$ NMR

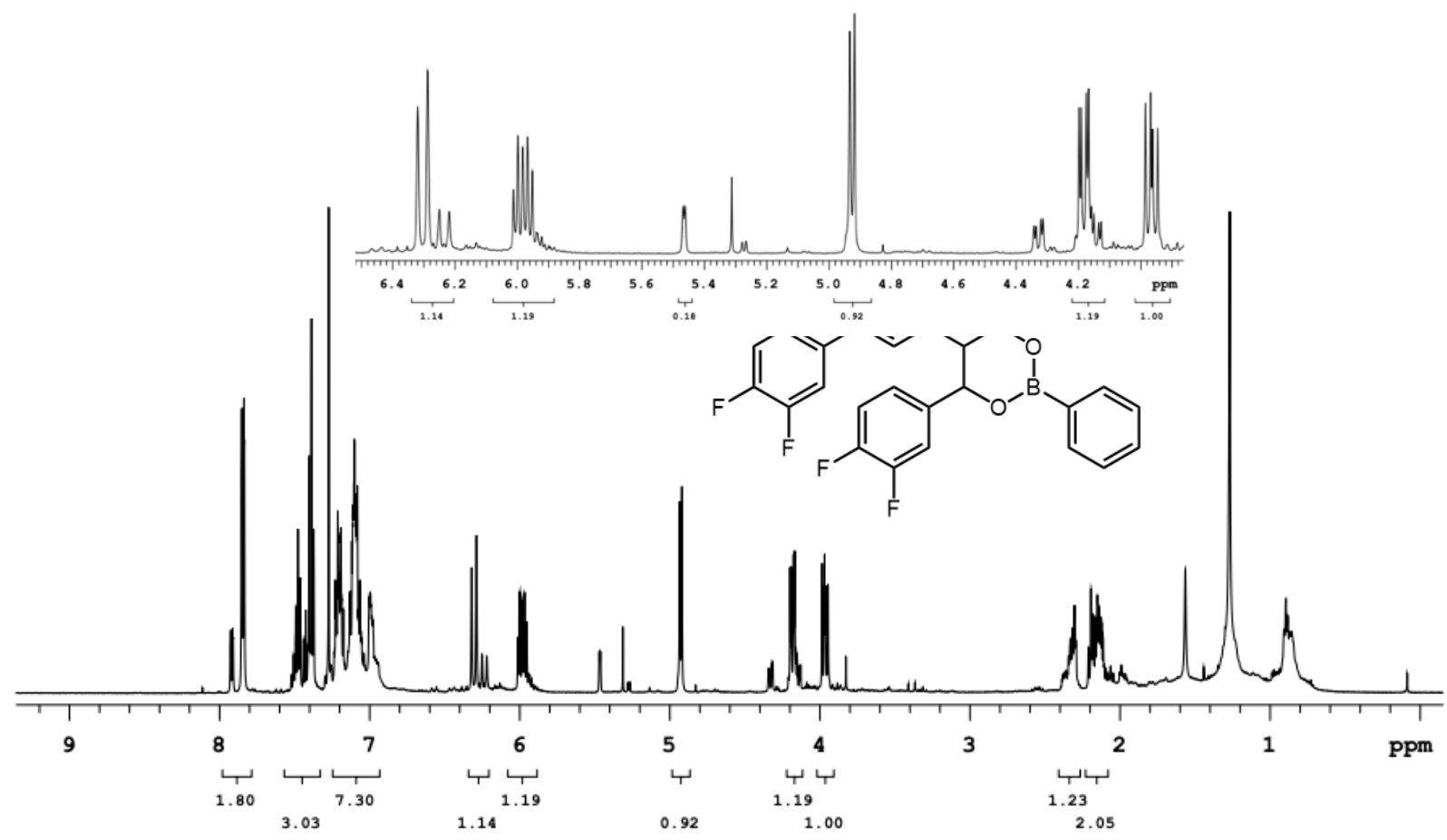

2q. (E)-4-(3,4-difluorophenyl)-5-(3-(3,4-difluorophenyl)allyl)-2-phenyl-1,3,2-dioxaborinane ${ }_{13} \mathrm{C}$ NMR

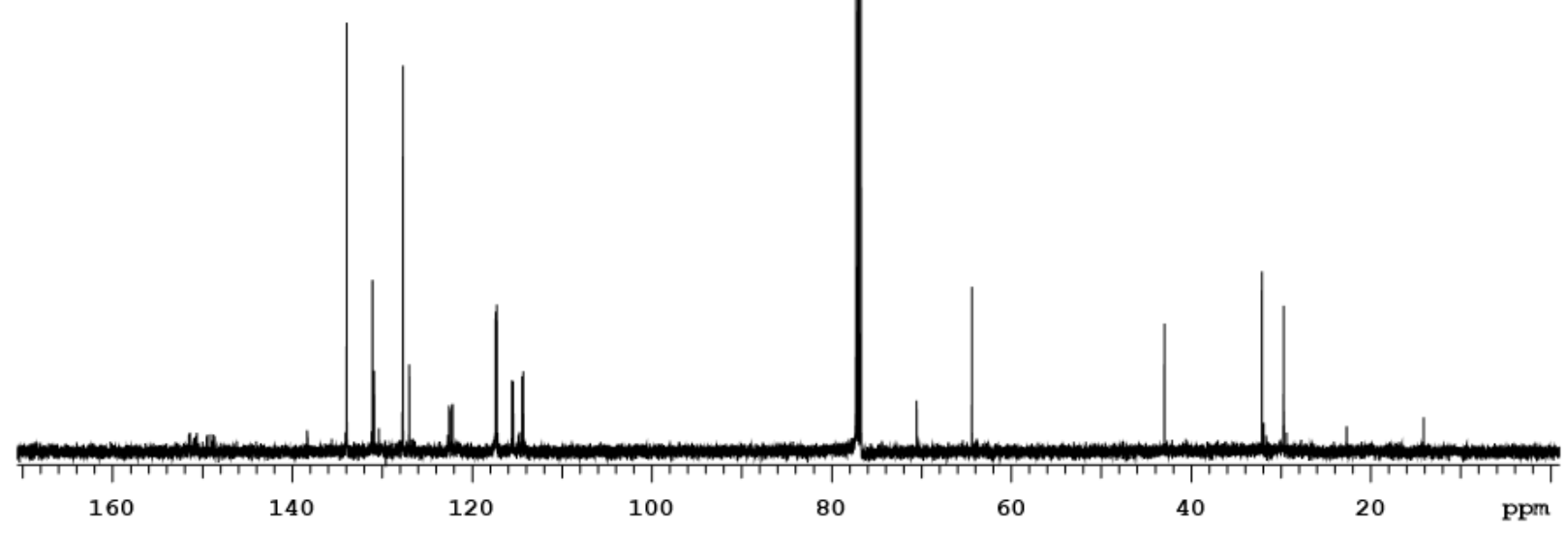


2r. (E)-2-phenyl-4-(thiophen-2-yl)-5-(3-(thiophen-2-yl)allyl)-1,3,2-dioxaborinane 1 H NMR

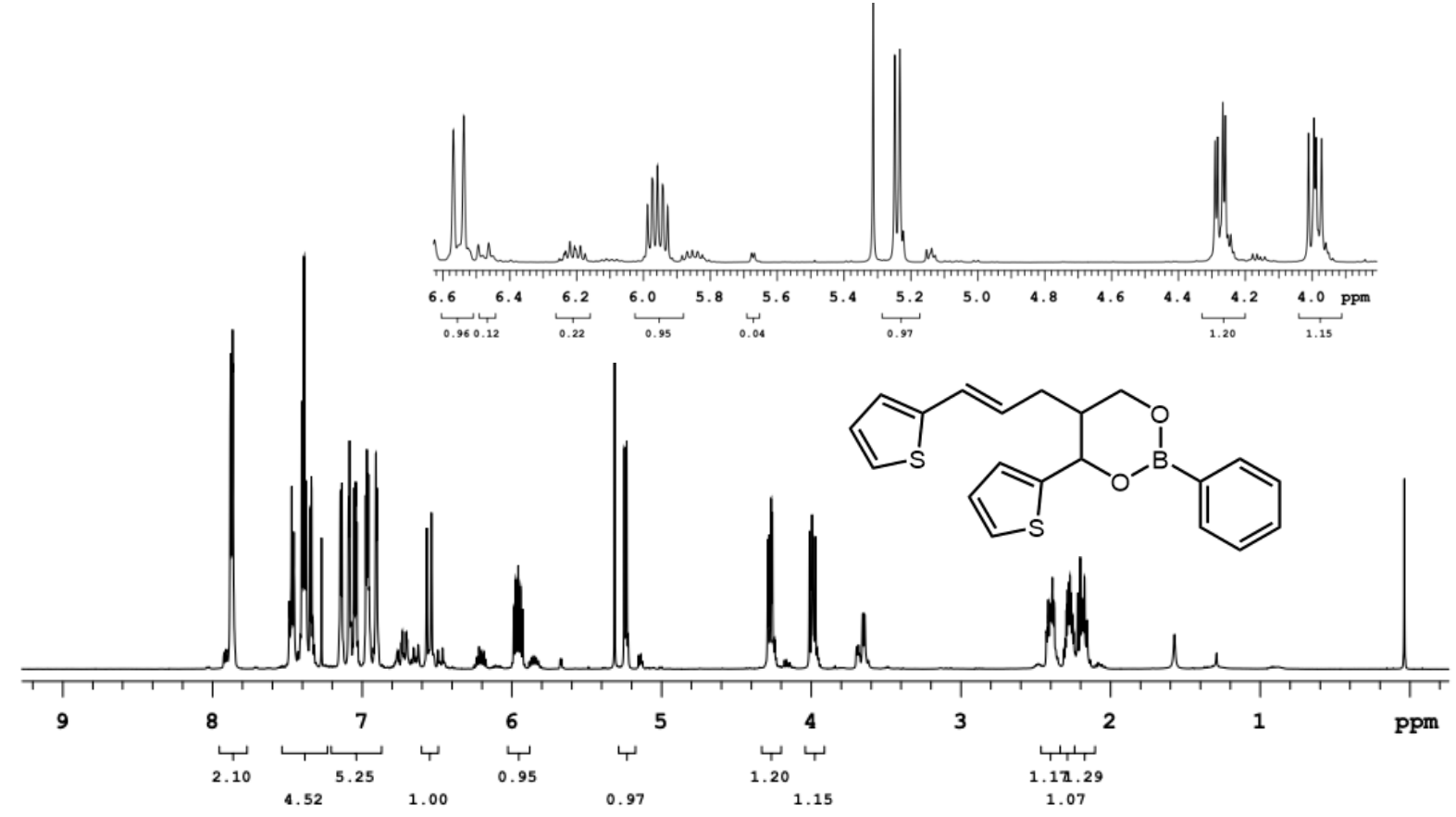

2r. (E)-2-phenyl-4-(thiophen-2-yl)-5-(3-(thiophen-2-yl)allyl)-1,3,2-dioxaborinane ${ }_{13} \mathrm{C}$ NMR

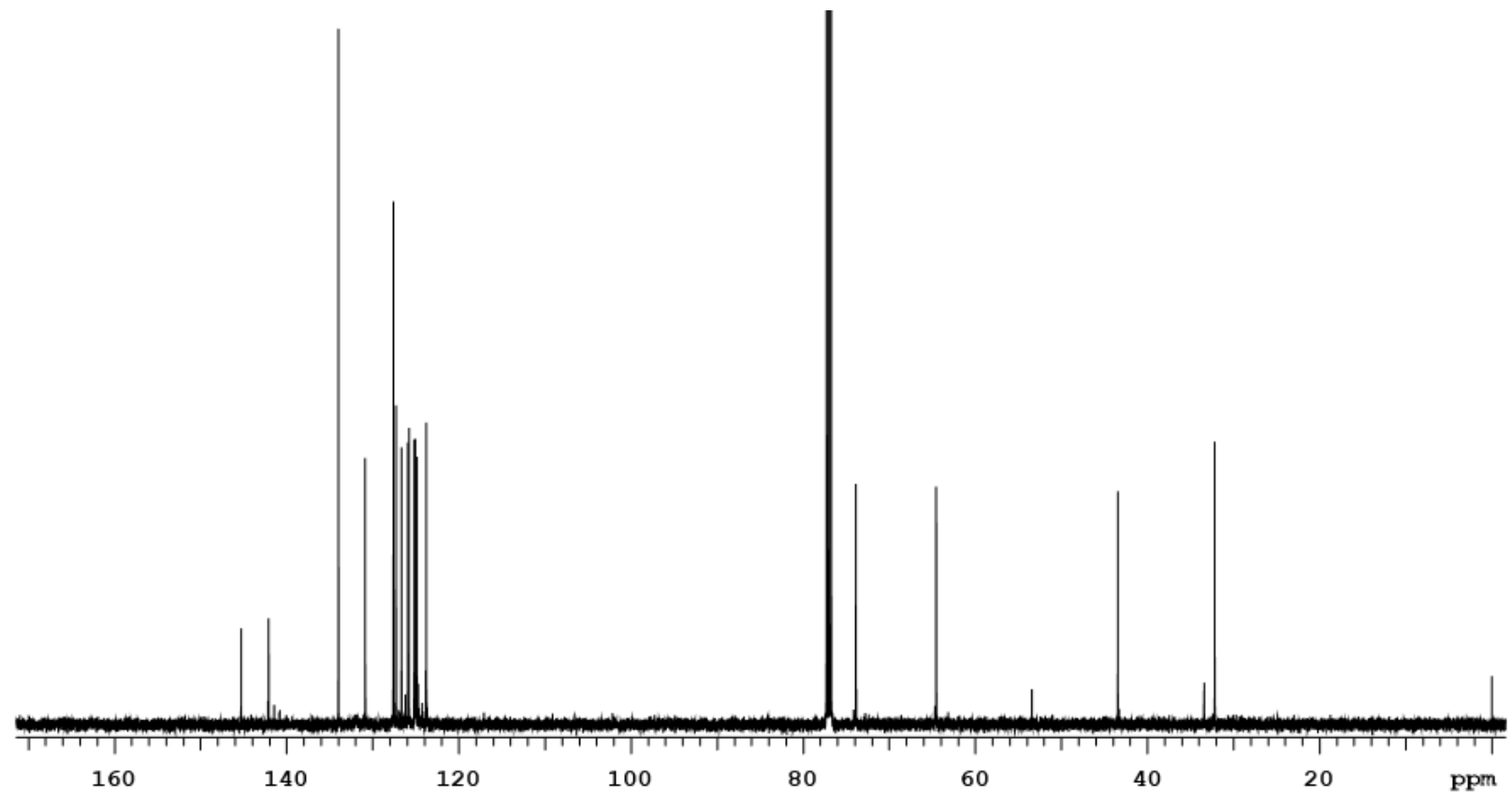


2s. 4,4-dimethyl-5-(3-methylbut-2-en-1-yl)-2-phenyl-1,3,2-dioxaborinane $1 \mathrm{H}$ NMR

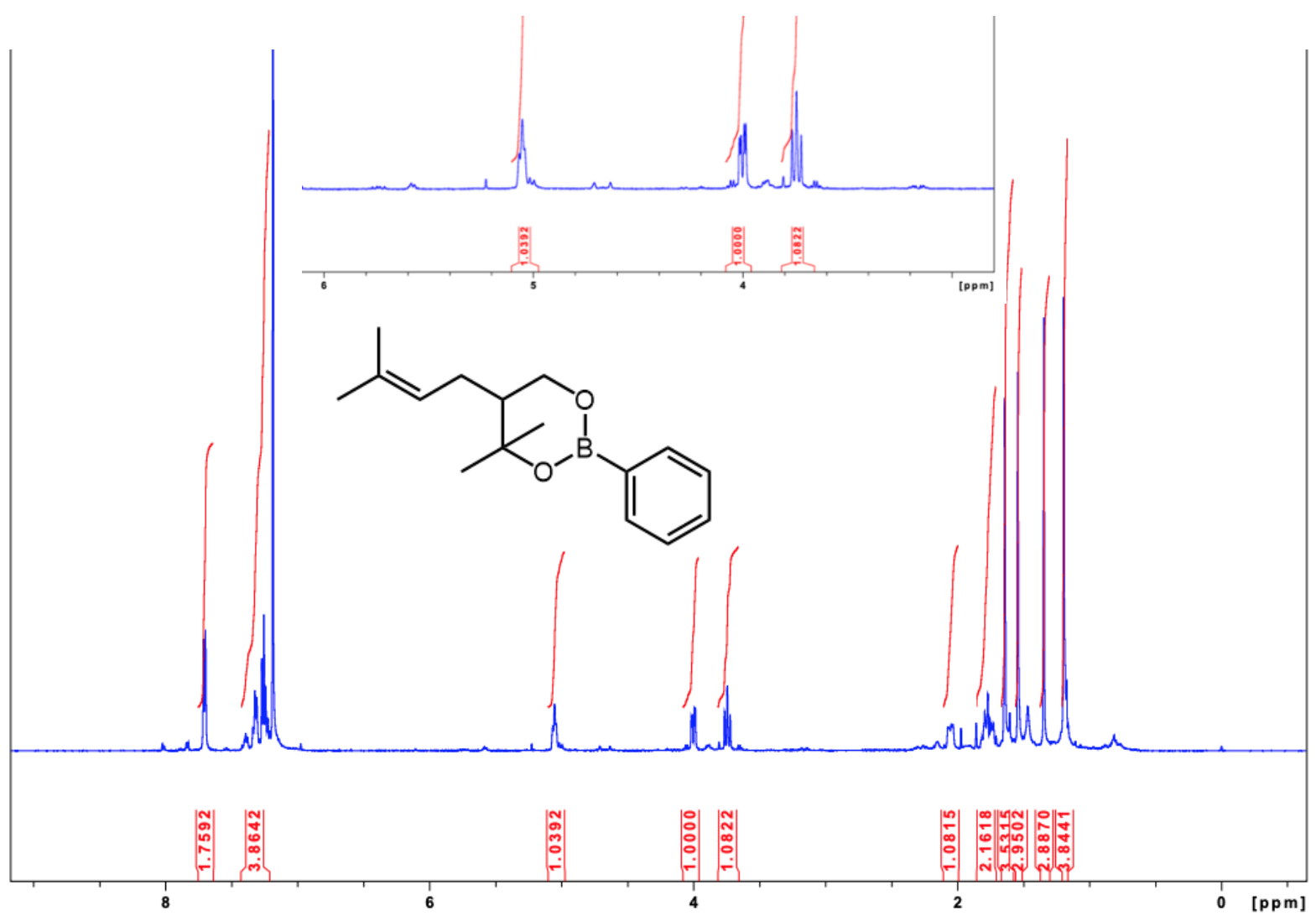

2s. 4,4-dimethyl-5-(3-methylbut-2-en-1-yl)-2-phenyl-1,3,2-dioxaborinane ${ }_{13} \mathrm{C}$ NMR

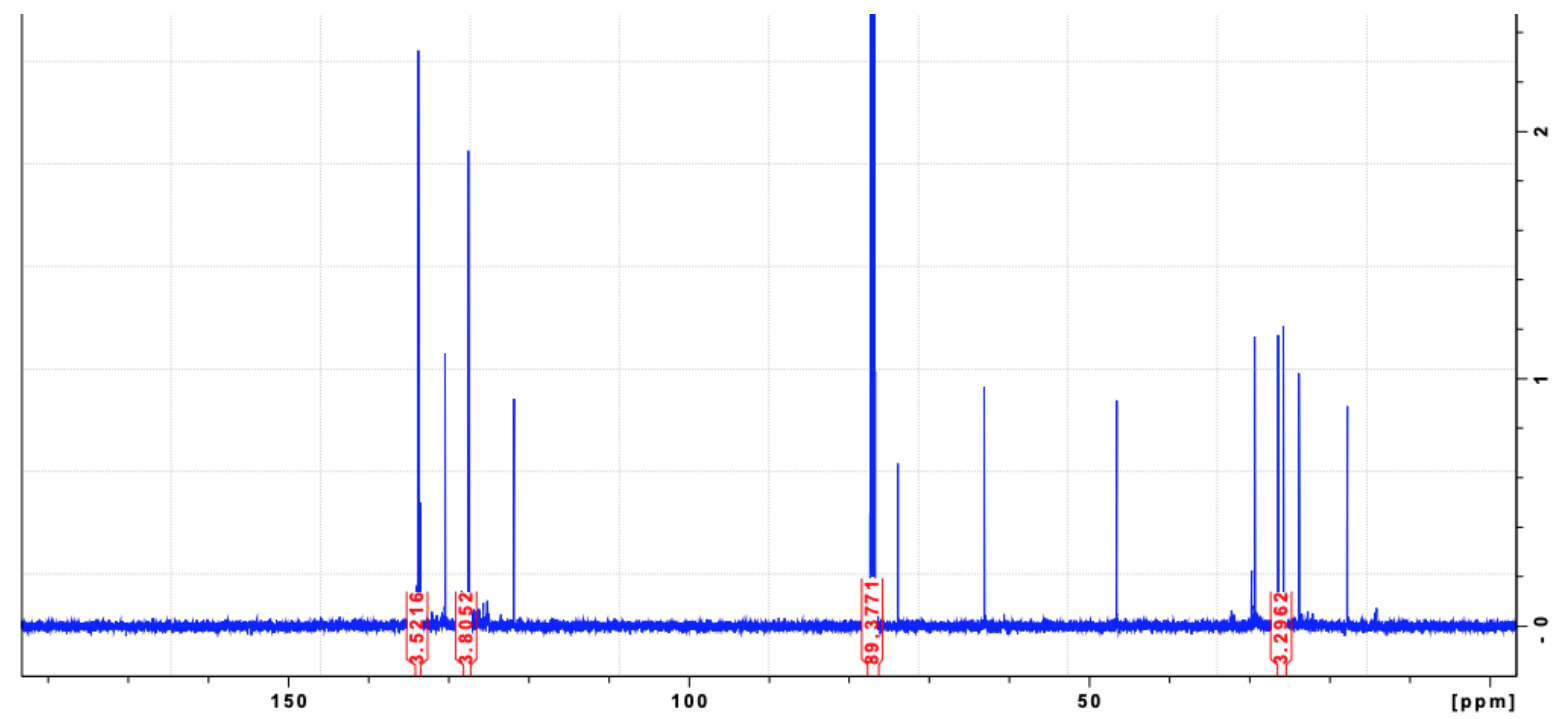


3. 2-cinnamyl-1-phenylpropane-1,3-diol ${ }_{1} \mathrm{H}$ NMR

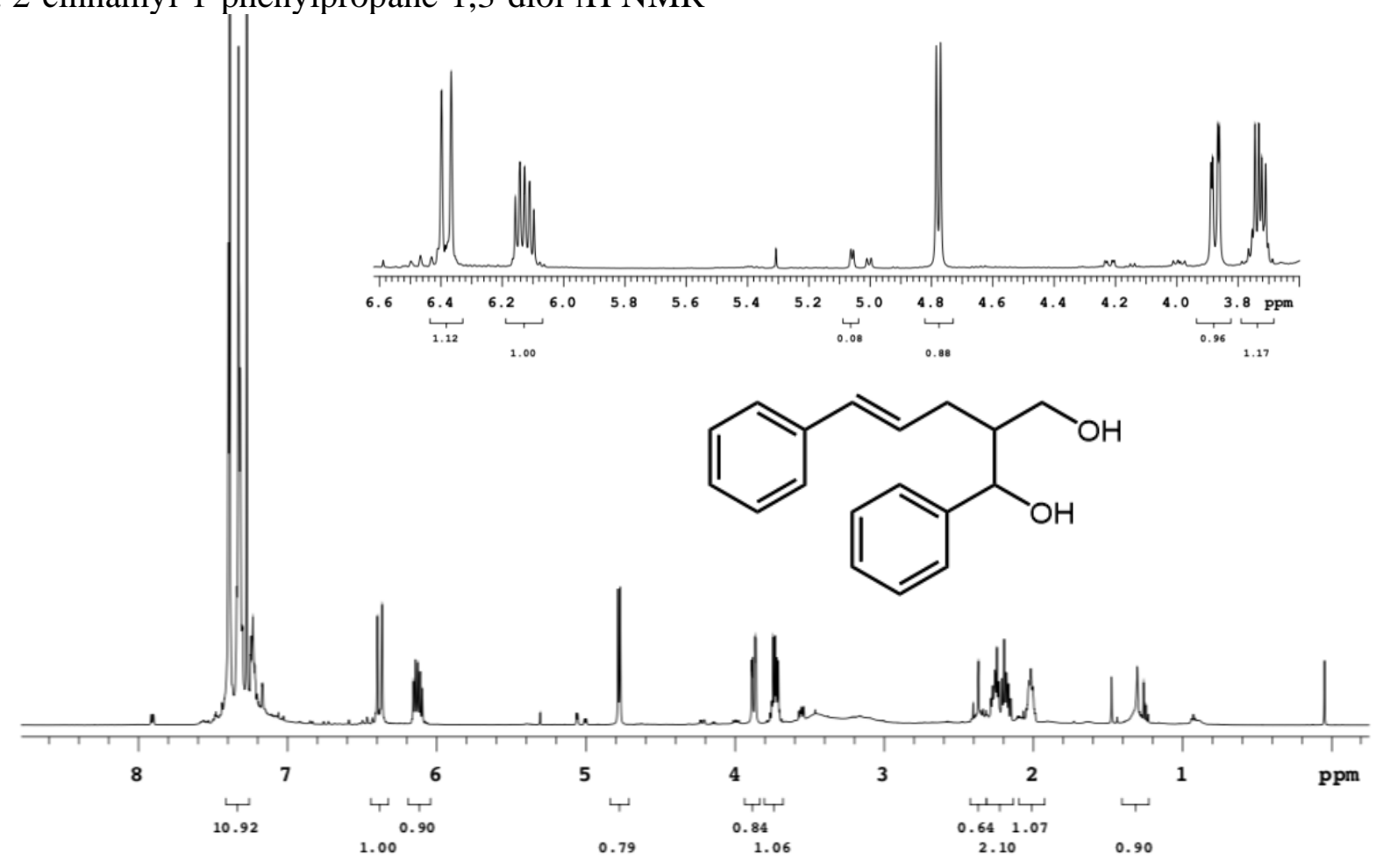

3. 2-cinnamyl-1-phenylpropane-1,3-diol 13C NMR

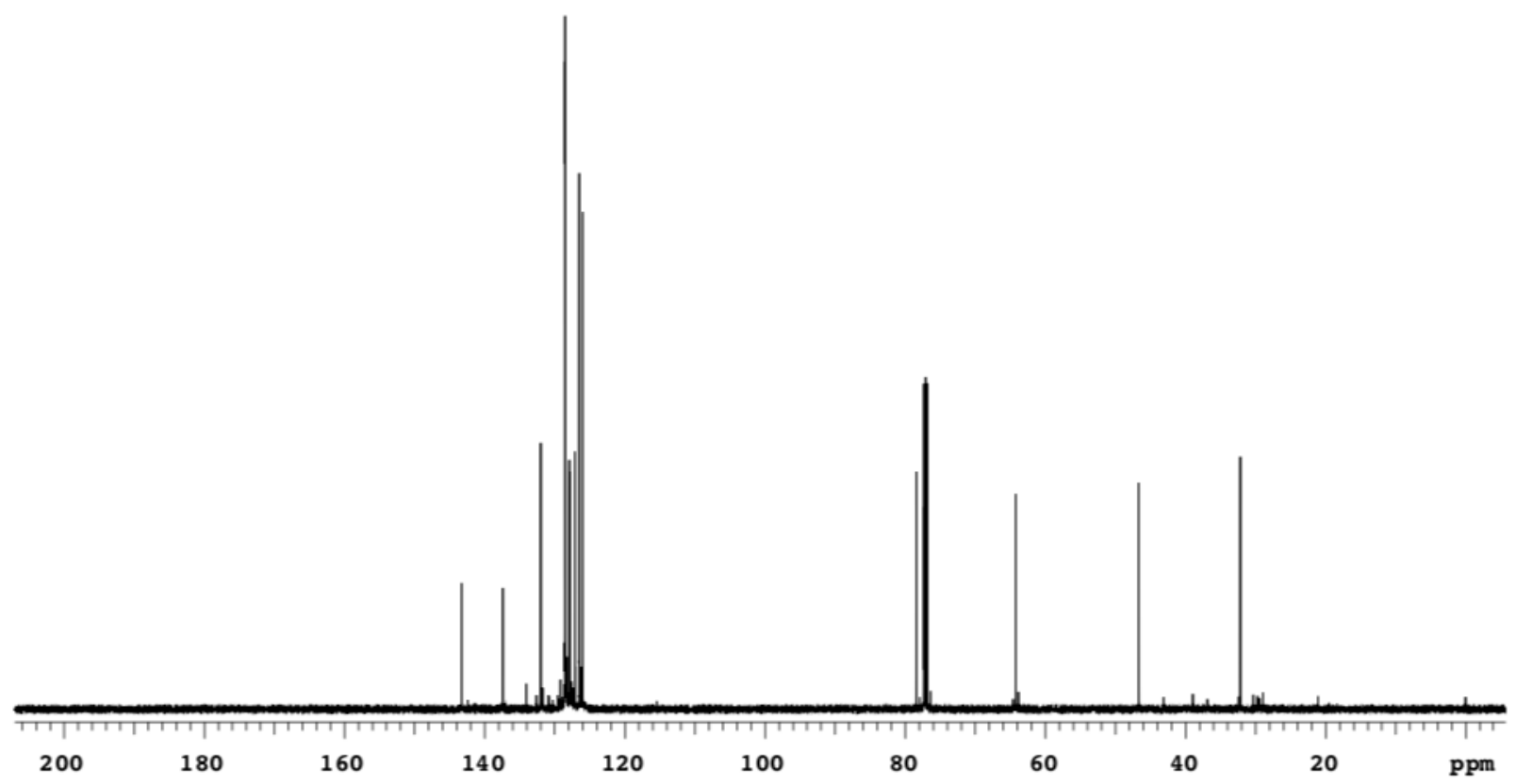




\section{Dicinnamyl phenylboronate $1 \mathrm{H}$ NMR}

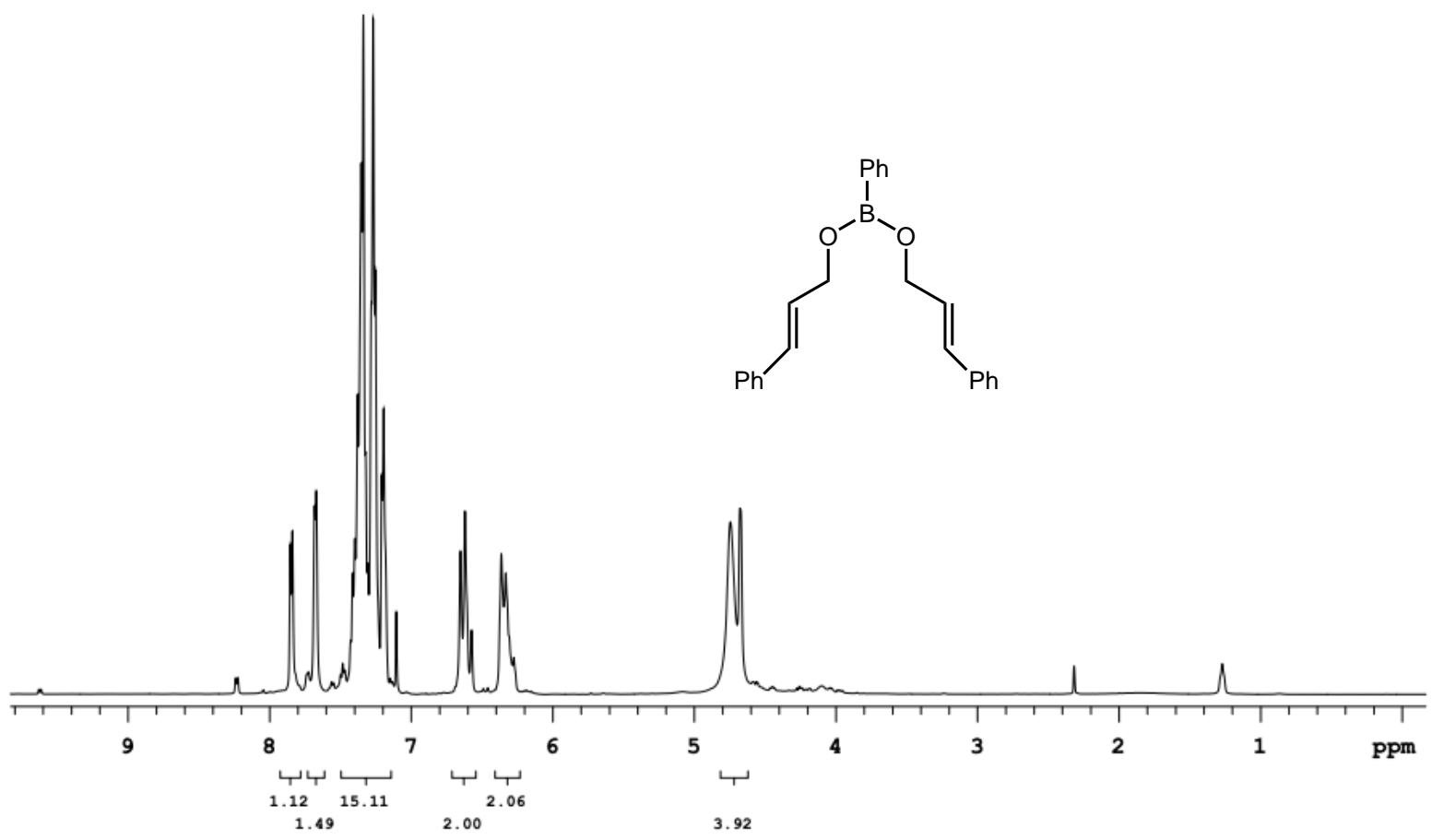

4. Dicinnamyl phenylboronate $13 \mathrm{C}$ NMR

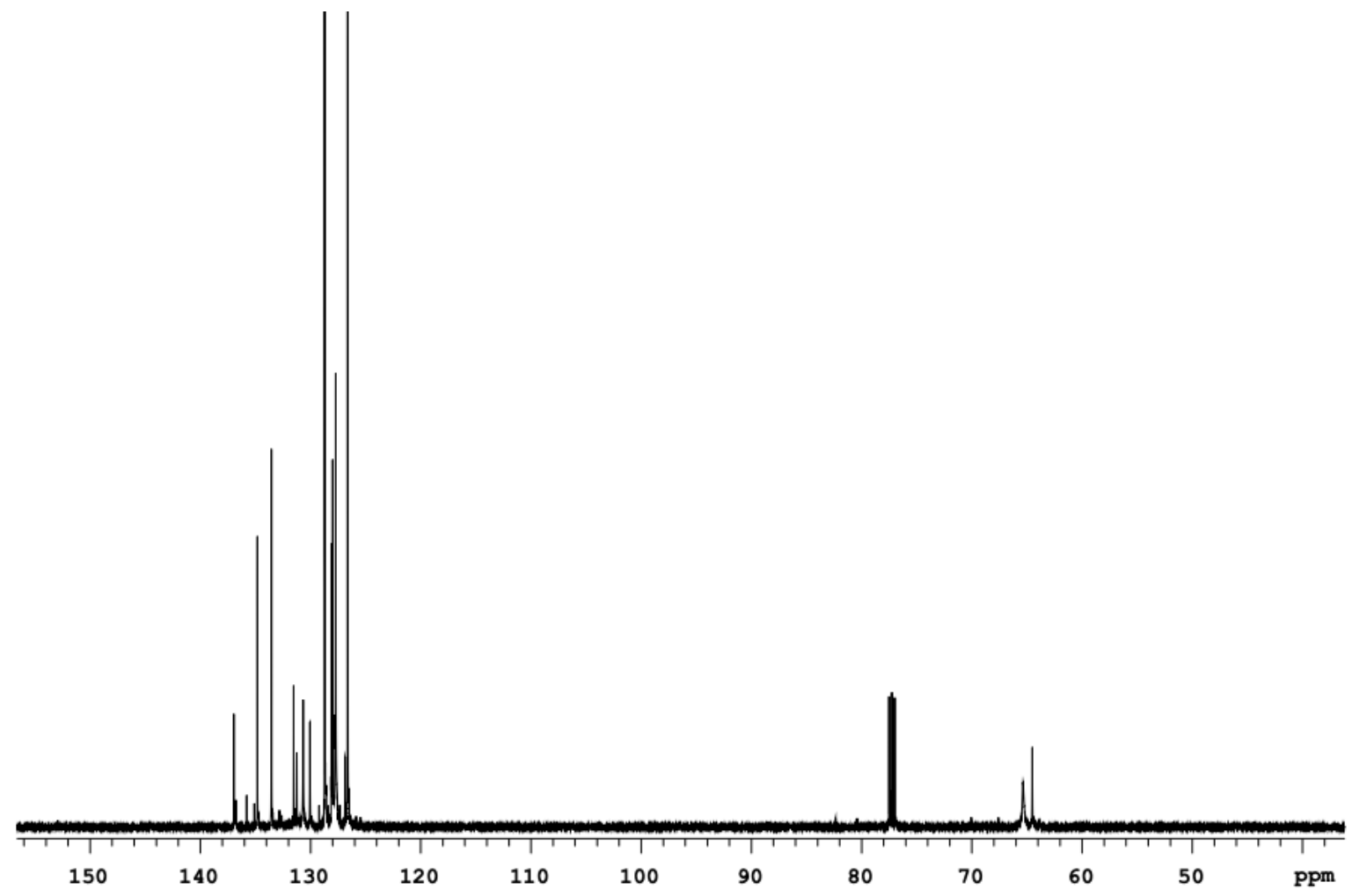


7. (4R,5R)-5-cinnamyl-4-phenyl-2-((R)-2'-phenyl-[1,1'-binaphthalen]-2-yl)-1,3,2-dioxaborinane ${ }_{1} \mathrm{H}$ NMR

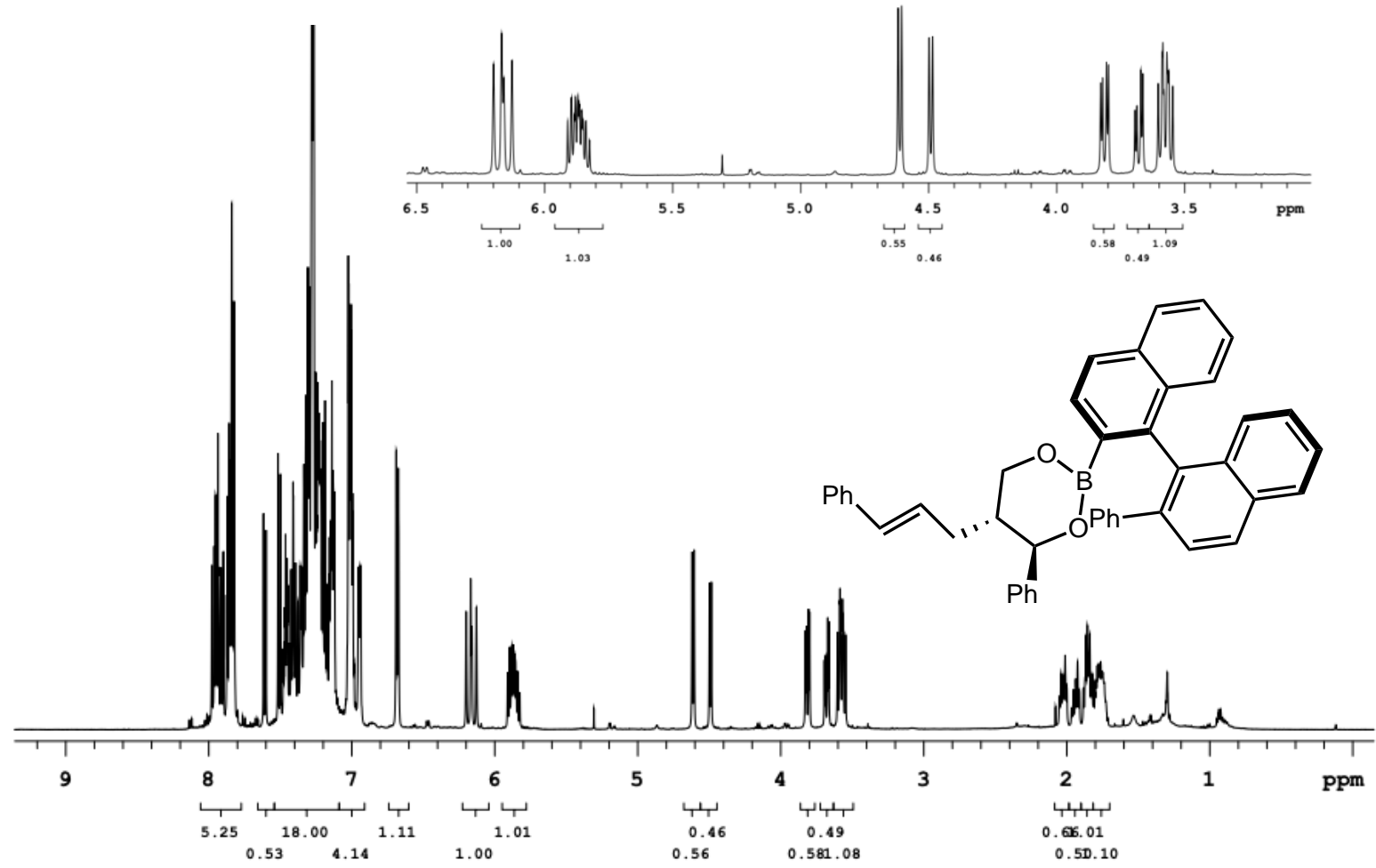

7. (4R,5R)-5-cinnamyl-4-phenyl-2-((R)-2'-phenyl-[1,1'-binaphthalen]-2-yl)-1,3,2-dioxaborinane ${ }_{13} \mathrm{C}$ NMR

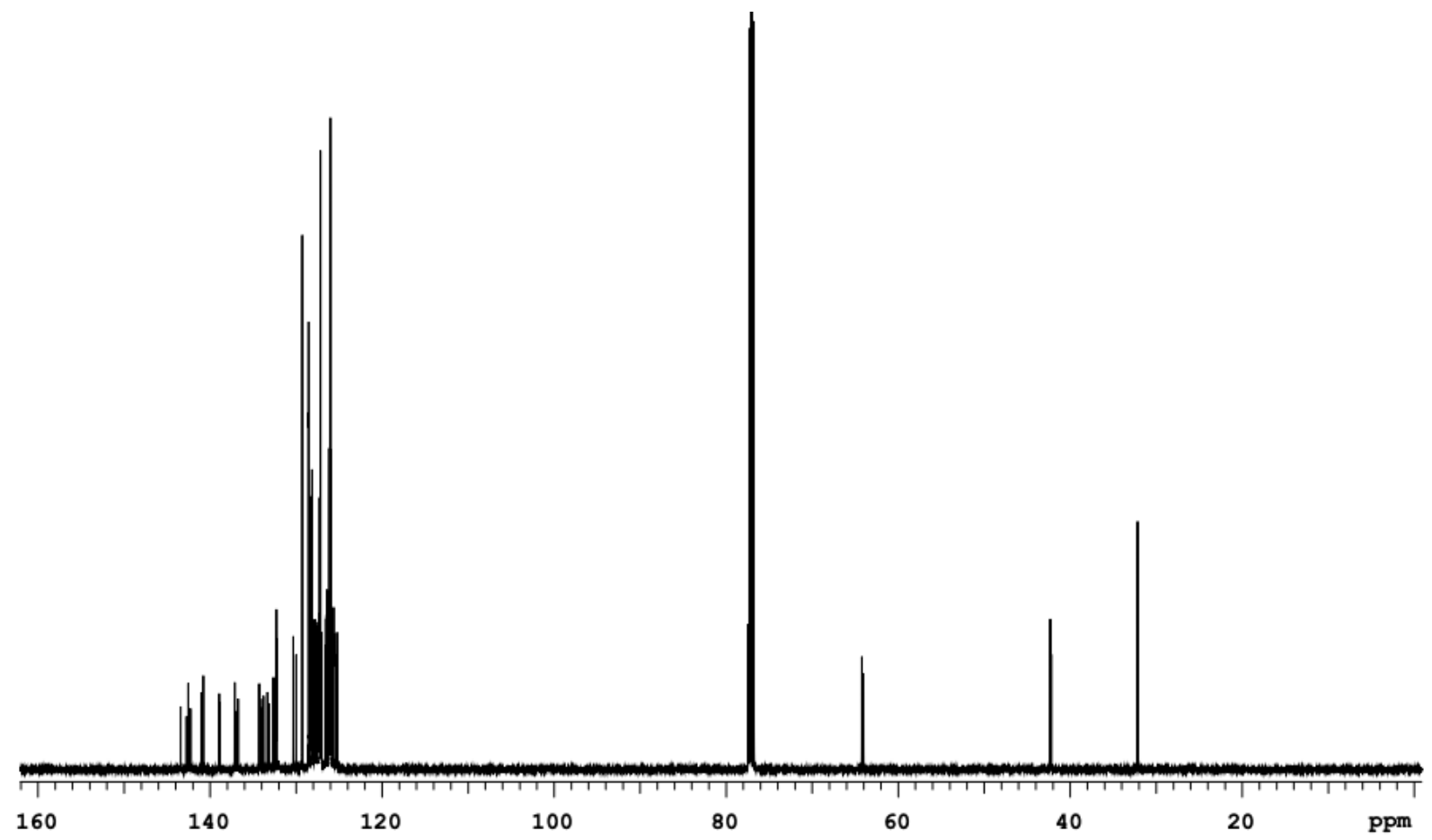


8. 2-(2,4-diphenyl-1,3,2-dioxaborinan-5-yl)acetaldehyde $1 \mathrm{H}$ NMR

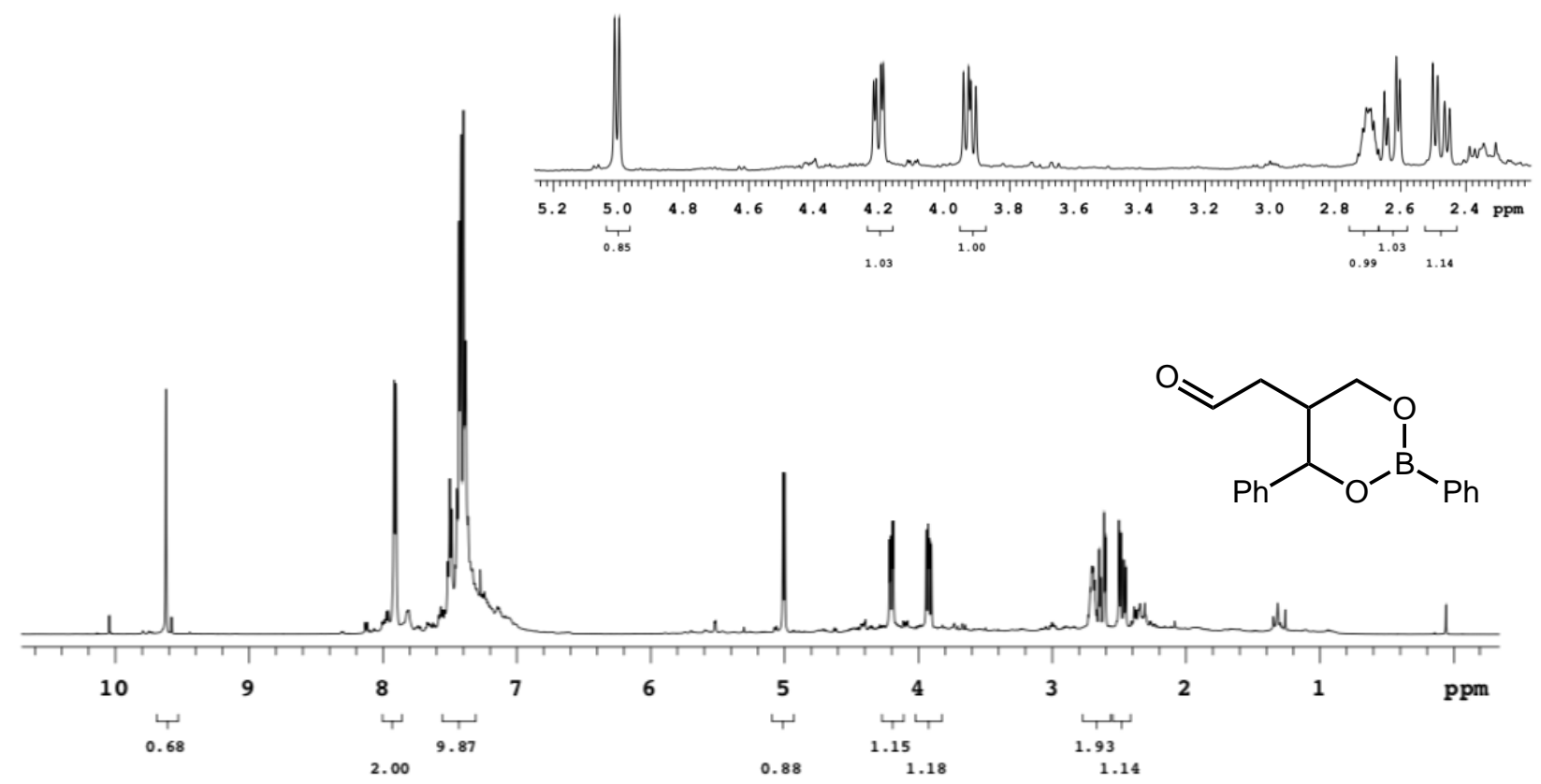

8. 2-(2,4-diphenyl-1,3,2-dioxaborinan-5-yl)acetaldehyde $13 \mathrm{C}$ NMR

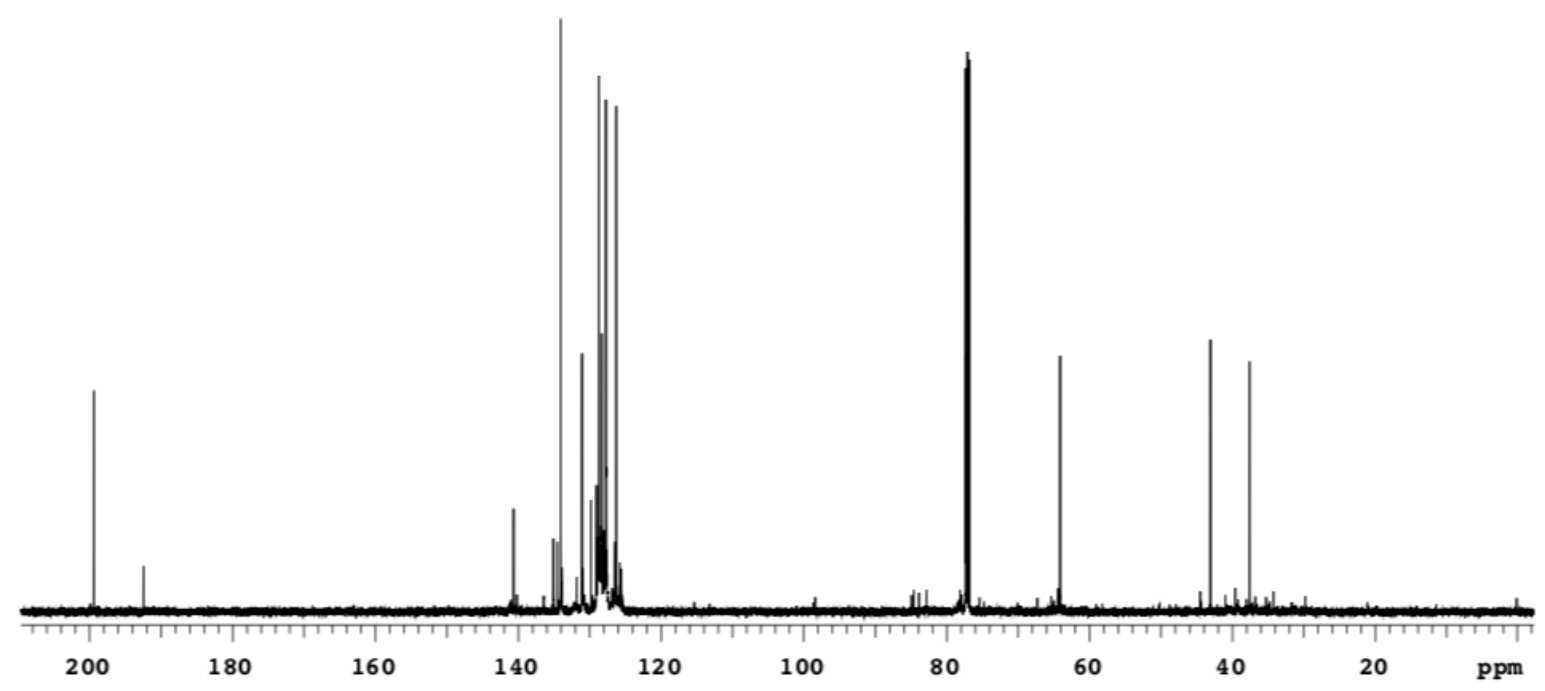


9. 2,4-diphenyl-5-(3-phenylpropyl)-1,3,2-dioxaborinane $1 \mathrm{H}$ NMR

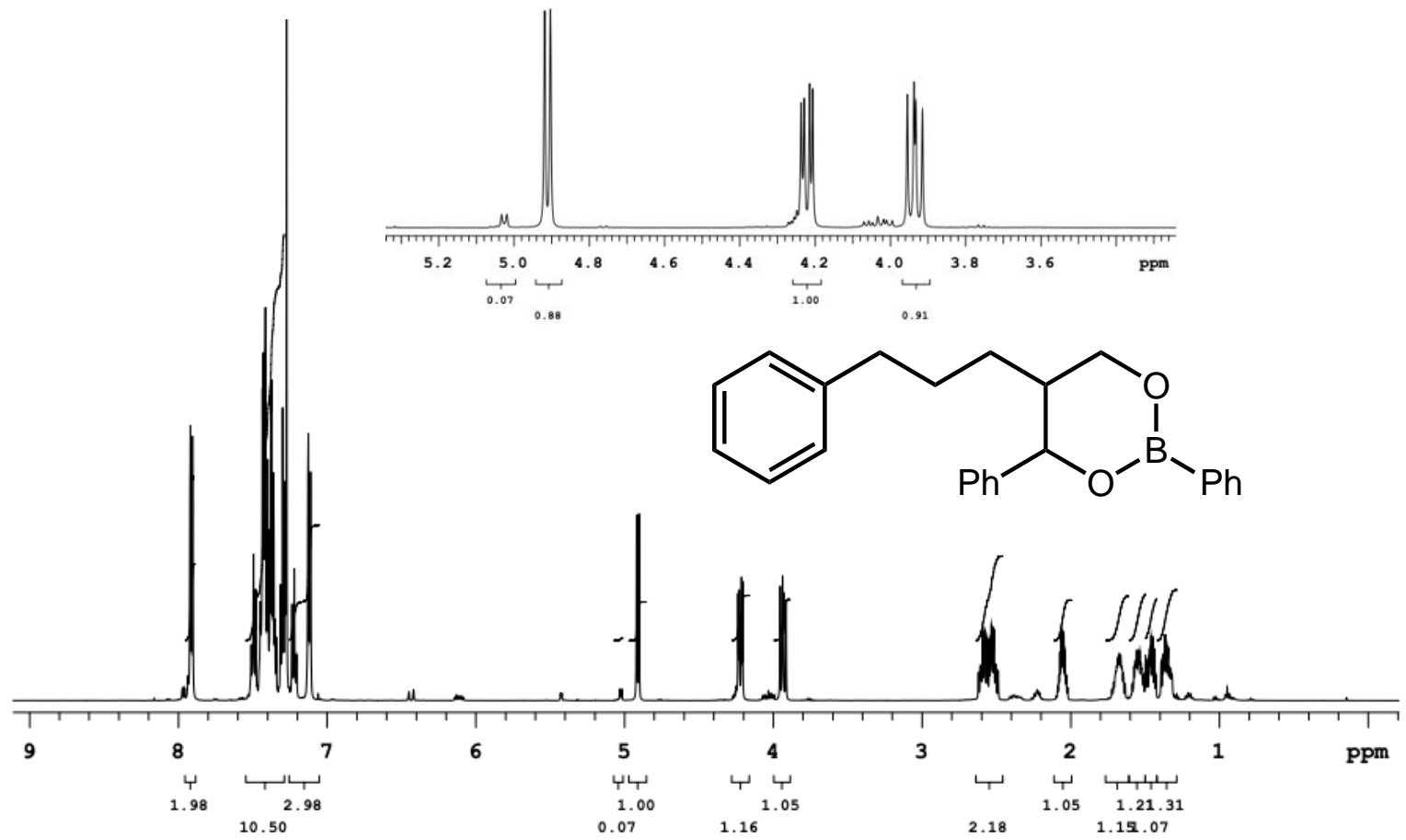

9. 2,4-diphenyl-5-(3-phenylpropyl)-1,3,2-dioxaborinane ${ }_{13} \mathrm{C}$ NMR

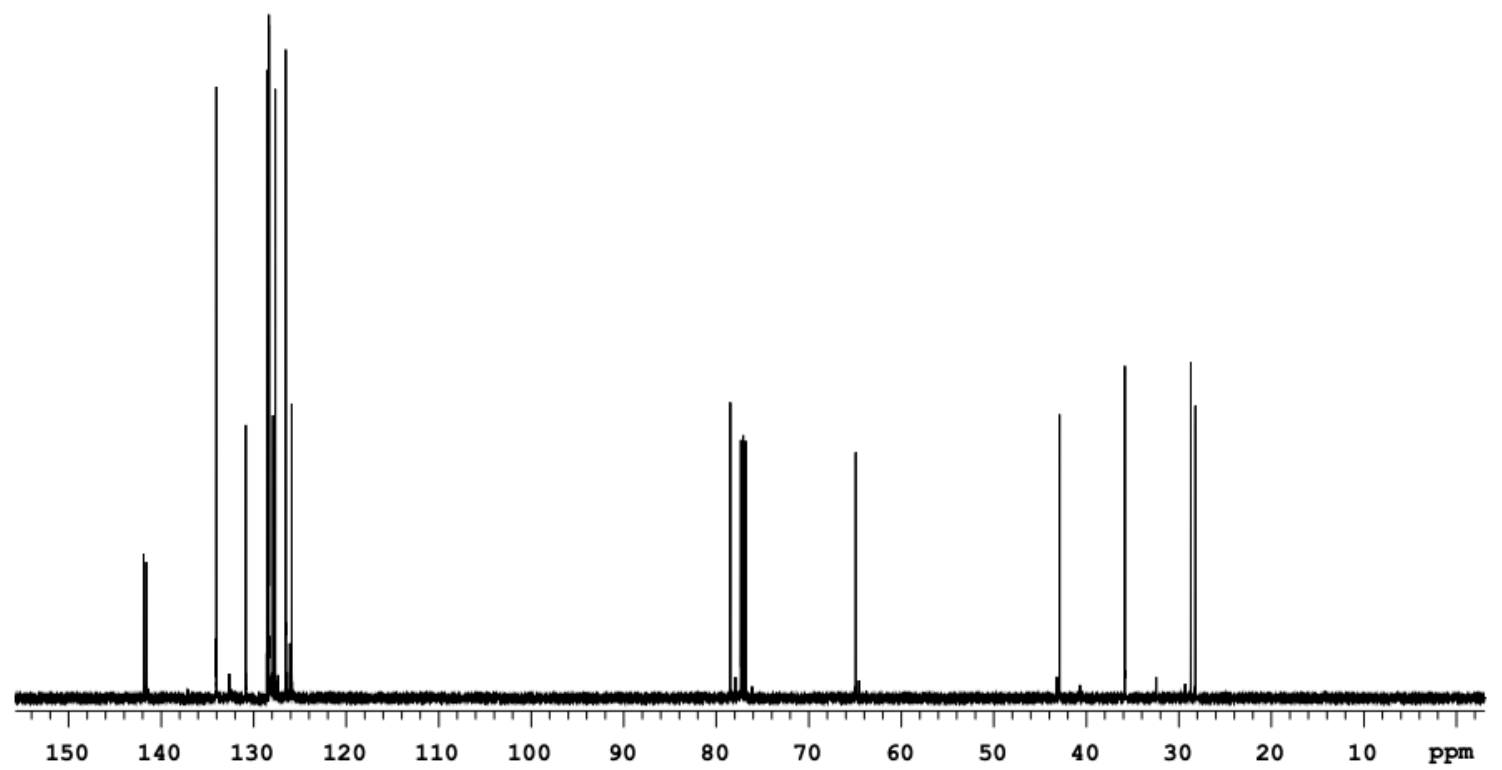


10. 5-allyl-2,4-diphenyl-1,3,2-dioxaborinane $1 \mathrm{H}$ NMR

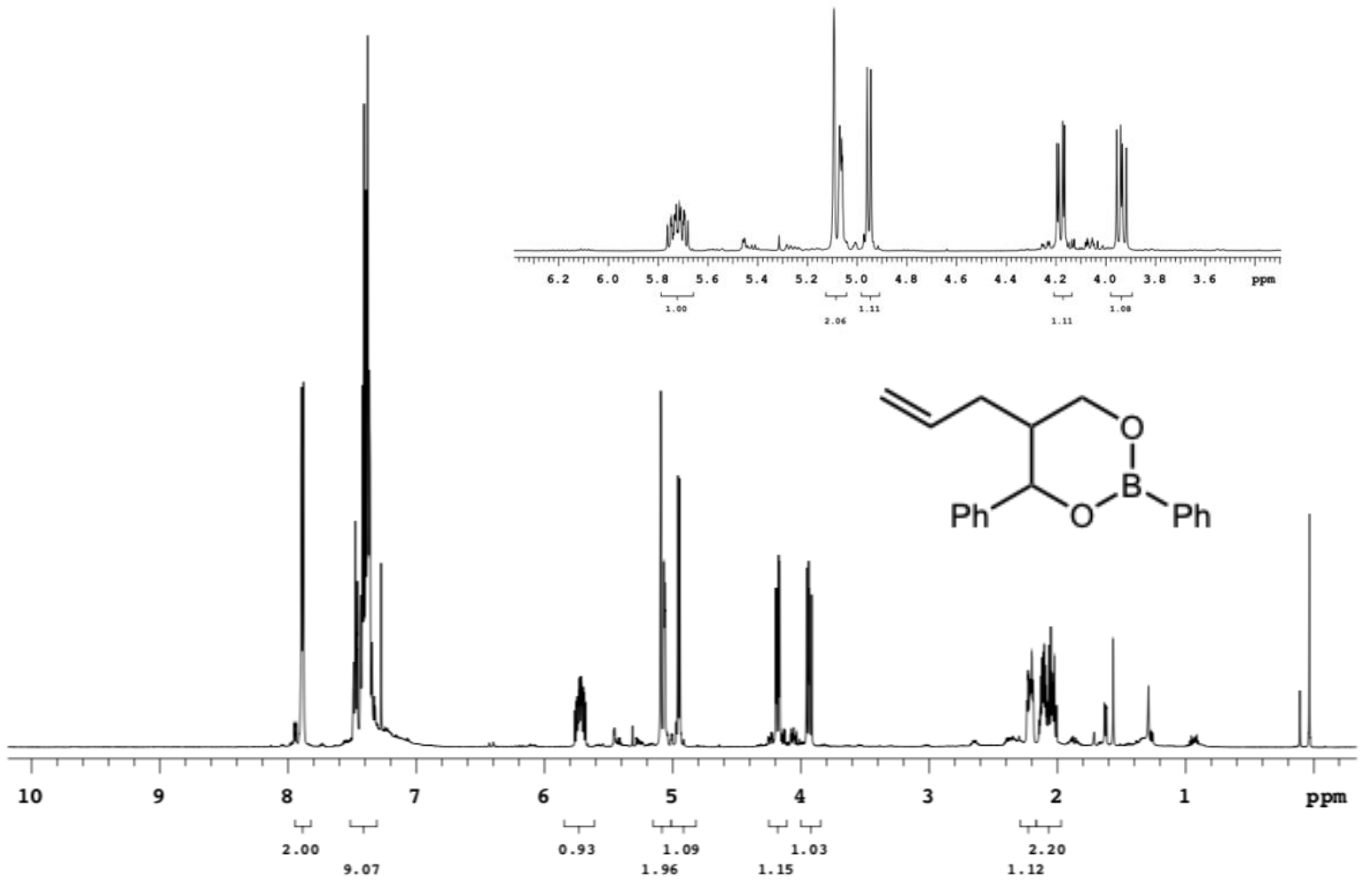

10. 5-allyl-2,4-diphenyl-1,3,2-dioxaborinane ${ }_{13} \mathrm{C}$ NMR

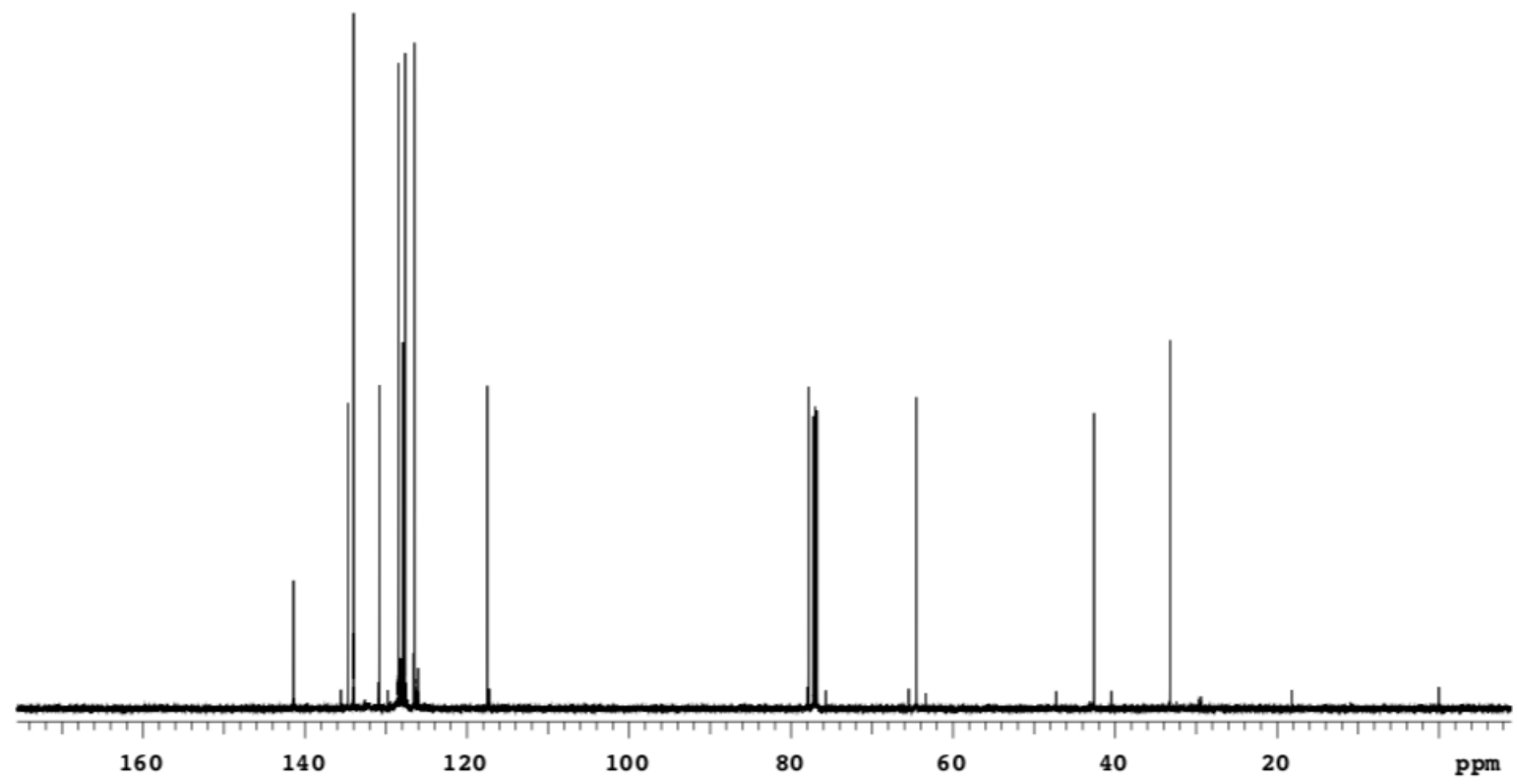


11. 2,4-diphenyl-5-((3-phenyloxiran-2-yl)methyl)-1,3,2-dioxaborinane $1 \mathrm{H}$ NMR

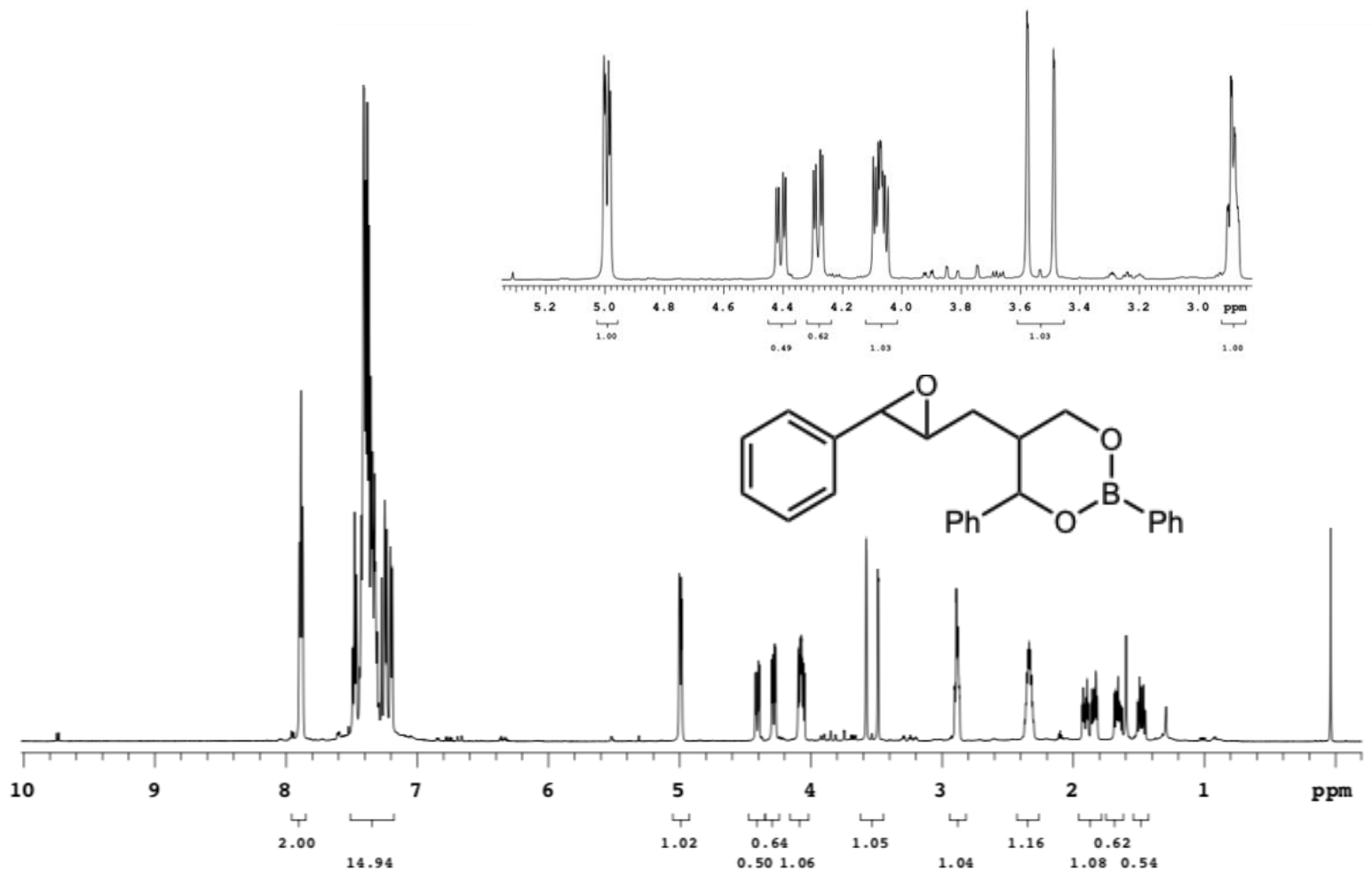

11. 2,4-diphenyl-5-((3-phenyloxiran-2-yl)methyl)-1,3,2-dioxaborinane ${ }_{13} \mathrm{C}$ NMR

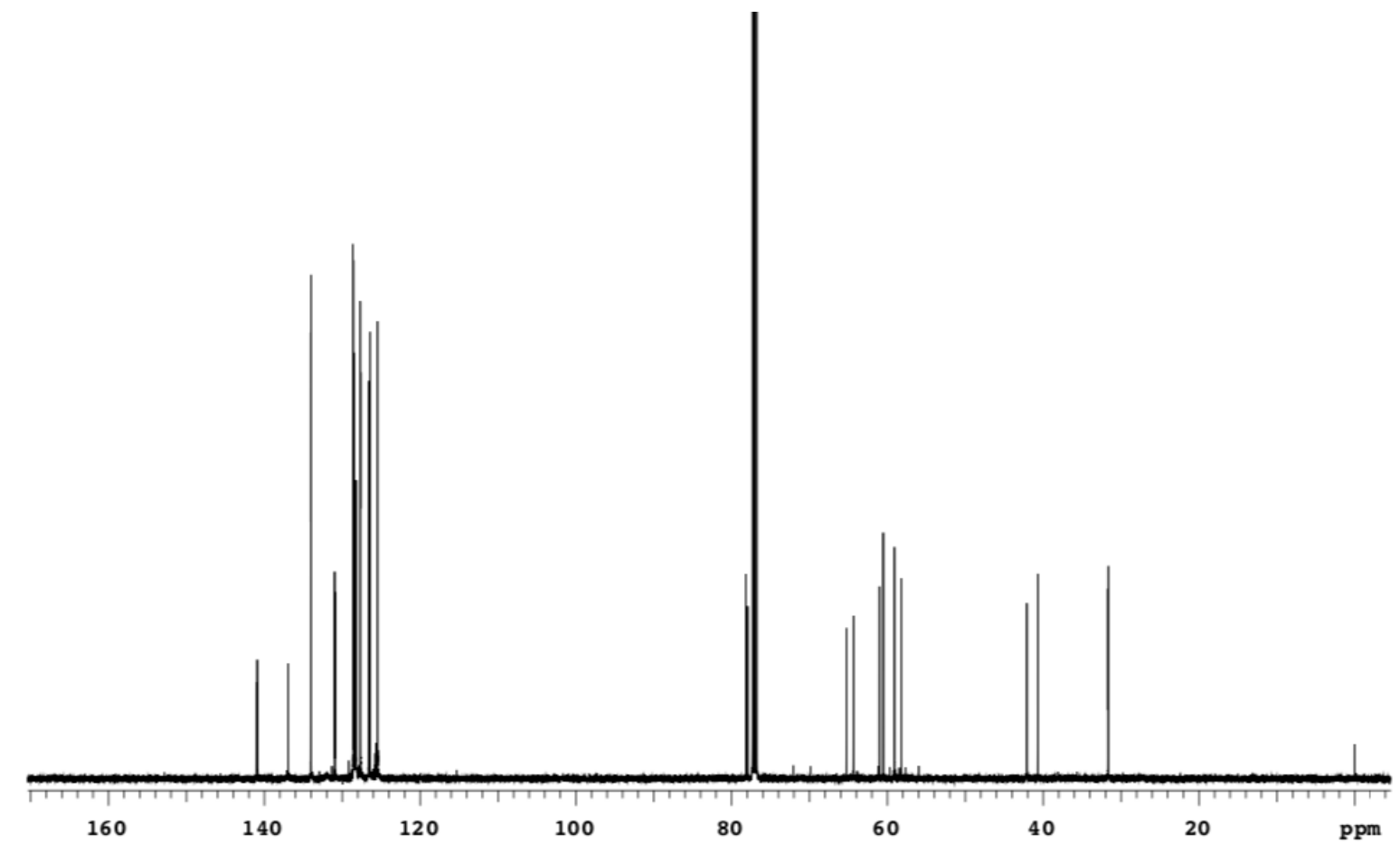

